\title{
THE YOUNG STELLAR POPULATION OF LYNDS 1340. AN INFRARED VIEW
}

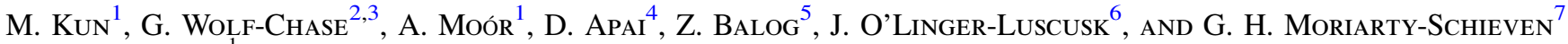 \\ ${ }^{1}$ Konkoly Observatory, Research Centre for Astronomy and Earth Sciences, Hungarian Academy of Sciences, \\ H-1121 Budapest, Konkoly Thege út 15-17, Hungary; kun@konkoly.hu \\ 2 Astronomy Department, Adler Planetarium, 1300 South Lake Shore Drive, Chicago, IL 60605, USA \\ ${ }^{3}$ Department of Astronomy and Astrophysics, University of Chicago, 5640 South Ellis Avenue, Chicago, IL 60637, USA \\ ${ }^{4}$ Steward Observatory, 933 N. Cherry Avenue, Tucson, AZ 85719, USA \\ ${ }^{5}$ Max-Planck-Institut für Astronomie, Königstuhl 17, D-69117 Heidelberg, Germany \\ ${ }^{6}$ On leave from California Institute of Technology, 1200 E. California Avenue, Pasadena, CA 91125, USA \\ ${ }^{7}$ National Research Council-Herzberg Astronomy \& Astrophysics, 5071 West Saanich Road, Victoria, BC V9E 2E7, Canada \\ Received 2015 December 16; accepted 2016 February 17; published 2016 June 1
}

\begin{abstract}
We present results of an infrared study of the molecular cloud Lynds 1340, forming three groups of low- and intermediate-mass stars. Our goals are to identify and characterize the young stellar population of the cloud, study the relationships between the properties of the cloud and the emergent stellar groups, and integrate L1340 into the picture of the star-forming activity of our Galactic environment. We selected candidate young stellar objects (YSOs) from the Spitzer and WISE databases using various published color criteria and classified them based on the slope of the spectral energy distribution (SED). We identified 170 Class II, 27 flat SED, and 45 Class 0/I sources. High angular resolution near-infrared observations of the RNO 7 cluster, embedded in L1340, revealed eight new young stars of near-infrared excess. The surface density distribution of YSOs shows three groups, associated with the three major molecular clumps of L1340, each consisting of $\lesssim 100$ members, including both premain-sequence stars and embedded protostars. New Herbig-Haro objects were identified in the Spitzer images. Our results demonstrate that L1340 is a prolific star-forming region of our Galactic environment in which several specific properties of the intermediate-mass mode of star formation can be studied in detail.
\end{abstract}

Key words: ISM: clouds - ISM: individual objects (L1340) - stars: formation - stars: pre-main sequence - stars: protostars

Supporting material: machine-readable tables

\section{INTRODUCTION}

The star-forming history of molecular clouds and the early evolution of stars and protoplanetary disks depend on the environment (e.g., Zhang \& Tan 2015). Since most stars form in a clustered environment, it is important to assess how this environment influences the timescales and efficiencies of star formation and the evolution of protoplanetary disks around young stars. The impact of feedback from the newborn highmass (spectral types $\mathrm{O}$ and early B) stars on the evolution of their natal cloud and the properties of the emergent star clusters are studied in detail by, e.g., Dib et al. (2013). Important basic properties of massive star-forming regions (MSFRs) have emerged from the MYStIX project (Feigelson et al. 2013). The effect of intermediate-mass stars (i.e., spectral types mid- to late $\mathrm{B}$ and early A) on the ambient medium in which they are forming has attracted less interest. There are clouds with structure and star-forming properties intermediate between the two extremes of isolated star formation (e.g., Taurus, Cepheus flare) and the rich clusters found around very massive stars (e.g., Orion). In these regions, young stars are concentrated in small clusters, whose highest-mass member is usually a B-type star. Well-known nearby examples of this type are IC 348, NGC 7023, and NGC 7129. The role of this intermediate mode of star formation in shaping the present appearance of our Galaxy is not well known. Adams \& Myers (2001) suggested that most of the Galactic stellar content might have originated from clusters containing fewer than some 100 members. A clearer observational picture of the intermediate mode is essential to our understanding of the star formation process.
Arvidsson et al. (2010) identified a sample of 50 intermediate-mass star-forming regions (IMSFRs), based on IRAS colors, Spitzer images, and millimeter continuum and ${ }^{13} \mathrm{CO}$ maps. They found typical luminosities of $\sim 10^{4} L_{\odot}$, diameters of $\sim 1 \mathrm{pc}$, and associated molecular clumps of mass $10^{3} M_{\odot}$. Recently, Lundquist et al. (2014) presented an all-sky sample of 984 candidate Galactic IMSFRs and studied in detail four of the candidates, confirming that these regions contain loose clusters of low- and intermediate-mass stars. The ${ }^{13} \mathrm{CO}$ survey of Lundquist et al. (2015) has shown that molecular line width and column density correlate with the infrared luminosity of the region. Several targets of the Spitzer survey of young stellar clusters within $1 \mathrm{kpc}$ of the Sun (Gutermuth et al. 2009) belong to this class of SFRs. Evidence for the impact of intermediatemass stars on their interstellar environment comes from Arce et al. (2011), who identified a great number of bubble-like structures in Perseus, most of them around intermediate-mass stars. Examination of these SFRs is particularly important because it helps us understand the relationship between cloud structure and star-forming mode.

The first large-scale study of Lynds 1340 (Kun et al. 1994, hereafter Paper I), including an objective prism survey for $\mathrm{H} \alpha$ emission, low-resolution ${ }^{12} \mathrm{CO},{ }^{13} \mathrm{CO}$, and $\mathrm{C}^{18} \mathrm{O}$ maps, and IRAS data analysis, suggests that this cloud is an IMSFR, containing a few mid-B, A, and early F-type stars associated with reflection nebulosities (Dorschner \& Gürtler 1968). The ${ }^{13} \mathrm{CO}$ maps revealed three clumps: L1340 A, L1340 B, and L1340 C. Ten dense cores have been identified in L1340 through a large-scale $\mathrm{NH}_{3}$ survey (Kun et al. 2003, hereafter Paper II), with masses and kinetic temperatures halfway 
between the values obtained for the ammonia cores in Taurus and Orion. Thirteen $\mathrm{H} \alpha$ emission objects were identified in Paper I, and 14, which were concentrated in the small nebulous cluster RNO 7 (Cohen 1980), were identified by Magakian et al. (2003). Herbig-Haro objects and their driving sources are reported in Kumar et al. (2003) and Magakian et al. (2003). An overview of the region is presented in Kun (2008). Our recent paper (Kun et al. 2015, hereafter Paper III) reports on 11 candidate intermediate-mass $\left(2-5 M_{\odot}\right)$ members and 58 new candidate $\mathrm{T}$ Tauri stars in L1340 and presents a revised distance of $825 \mathrm{pc}$.

Whereas most of the cluster-forming molecular clouds of our Galactic neighborhood, including those studied by Arvidsson et al. (2010) and Lundquist et al. (2014), are parts of giant SFRs, which also contain high-mass stars (e.g., Ridge et al. 2003), Lynds 1340 is an isolated molecular cloud of some $3700 M_{\odot}$ at a Galactic latitude of $b \approx 11.5$, corresponding to some $160 \mathrm{pc}$ distance above the Galactic plane. To explore the nature of interstellar processes, leading to star formation in this environment, the cloud structure and the young stellar population have to be mapped. In this paper we identify the young stellar object (YSO) population of L1340 based on Spitzer and WISE midinfrared data, as well as on high angular resolution near-infrared imaging data of the embedded RNO 7 cluster. The goals of our studies are as follows: (i) determine the properties of star formation in this cloud, such as surface distribution, mass and age spread, accretion and disk properties of young stars, and efficiency of star formation; (ii) explore possible feedback from intermediate-mass stars; and (iii) integrate this cloud into the picture of star formation of our $1 \mathrm{kpc}$ Galactic environment. We describe the available data and analysis in Section 2. The results are presented and discussed in Sections 3-6. A short summary of the results is given in Section 7.

\section{DATA}

\subsection{Spitzer Data}

L1340 was observed by the Spitzer Space Telescope using Spitzer's Infrared Array Camera (IRAC; Fazio et al. 2004) on 2009 March 16 and the Multiband Imaging Photometer for Spitzer (MIPS; Rieke et al. 2004) on 2008 November 26 (Prog. ID: 50691, PI: G. Fazio). The IRAC observations covered $\sim 1 \mathrm{deg}^{2}$ in all four bands. Moreover, a small part of the cloud, centered on RNO 7, was observed in the four IRAC bands on 2006 September 24 (Prog. ID: 30734, PI: D. Figer). Figure 1 shows the areas of the Spitzer observations, overplotted on the DSS2 red image of the region. ${ }^{13} \mathrm{CO}$ contours from Paper I are drawn to indicate the boundaries of the molecular cloud, and the L1340 A, L1340, and L1340 C clumps are marked. The centers of the 3.6 and $5.8 \mu \mathrm{m}$ images are slightly displaced from those of the 4.5 and $8 \mu \mathrm{m}$ images; therefore, part of the clump L1340 C is outside of the 4.5 and $8 \mu \mathrm{m}$ maps. Moreover, the 24 and $70 \mu \mathrm{m}$ images do not cover the southern half of L1340 A. The data of the four IRAC and MIPS $24 \mu \mathrm{m}$ bands were processed by the Spitzer Science Center (SSC), and the resulting Super Mosaics and Source List are available at http://irsa.ipac.caltech.edu/data/ SPITZER/Enhanced/SEIP/. We selected candidate YSOs from the Spitzer Enhanced Imaging Products (SEIP) Source List, containing 19,745 point sources in the target field.

We followed the methods described in Gutermuth et al. (2009) for removing probable extragalactic, stellar, and interstellar sources and selecting candidate YSOs based on

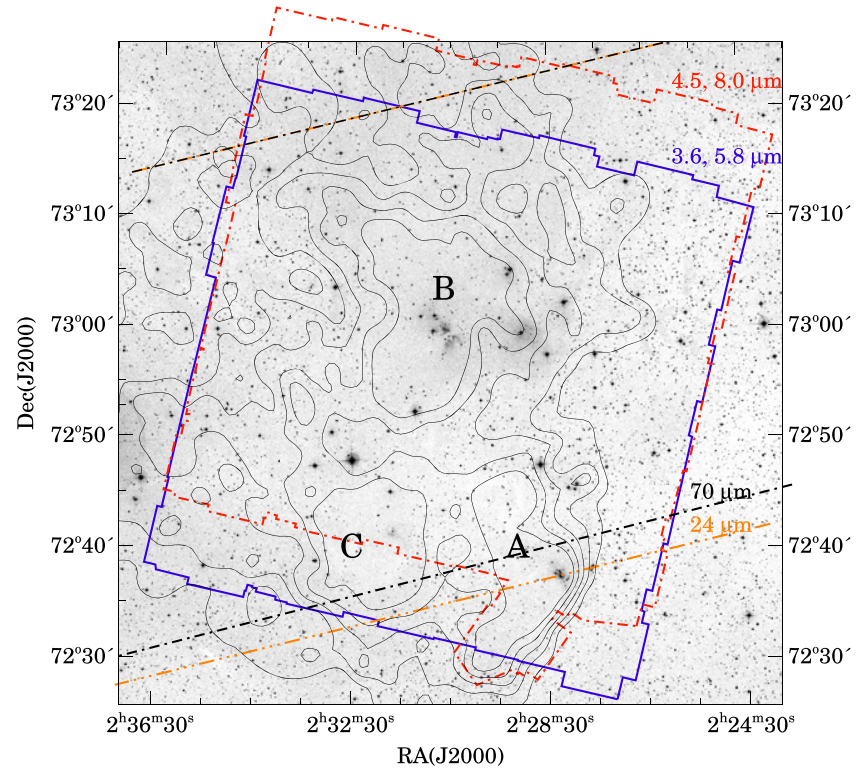

Figure 1. Outlines of the Spitzer IRAC (blue solid line: 3.6 and $5.8 \mu \mathrm{m}$; red dot-dashed line: 4.5 and $8.0 \mu \mathrm{m}$ ), MIPS $24 \mu \mathrm{m}$ (orange triple-dot-dashed line), and MIPS $70 \mu \mathrm{m}$ (black dot-dashed line) observations, overplotted on the DSS2 red image of the region, 1 square degree in area. Both the lowest contour and the increment of the overlaid ${ }^{13} \mathrm{CO}$ integrated intensity map are $0.5 \mathrm{~K} \mathrm{~km} \mathrm{~s}^{-1}$

color indices. We identified 98 candidate YSOs detected in each of the four IRAC bands (Phase 1 criteria of Gutermuth et al. 2009). Phase 2 criteria, based on Two Micron All Sky Survey (2MASS), 3.6 and $4.5 \mu \mathrm{m}$ data, resulted in 44 new YSO candidates. Based on their high MIPS $24 \mu \mathrm{m}$ fluxes and very red [24]-[IRAC ${ }_{i}$ ] color (Phase 3 criteria), we identified 46 additional sources that were missing one or more IRAC band data. Four additional sources obeyed the criteria [4.5][8.0] $>0.5$ and [8.0] $<14-([4.5]-[8.0])$, set by Harvey et al. (2006). A sizeable area of the cloud was observed only at 3.6 and $5.8 \mu \mathrm{m}$. We regarded sources, located in this area and having [3.6]-[5.8] $>0.50$, as candidate YSOs. Thirteen new objects were selected by this criterion. Most of them have associated Sloan Digital Sky Survey (SDSS), 2MASS, and/or WISE data, which help confirm their candidate YSO nature. We also subjected the SEIP Source List of L1340 to the criteria established by Kryukova et al. (2012) for selecting protostars. Of the 116 sources meeting the color criteria, there are 19 not selected during the previous steps and located within the lowest significant $\mathrm{C}^{18} \mathrm{O}$ contours of the cloud clumps. These sources were also included in the candidate YSO list.

Owing to the strict quality requirements of the SEIP Source List, several sources might have been missed in one or more bands. Furthermore, the $70 \mu \mathrm{m}$ data are not included in the SEIP database. Therefore, we checked the positions of the selected sources and performed photometry by the procedures described in Kun et al. (2014) to refill the missing flux data. Then we checked the $70 \mu \mathrm{m}$ images at each source position and measured $70 \mu \mathrm{m}$ fluxes. Figure 2 compares our photometry with the SEIP Source List data.

\subsection{High Angular Resolution Near-infrared Imaging}

High angular resolution near-infrared images of two small regions of L1340 were obtained on 2002 October 24 in the 


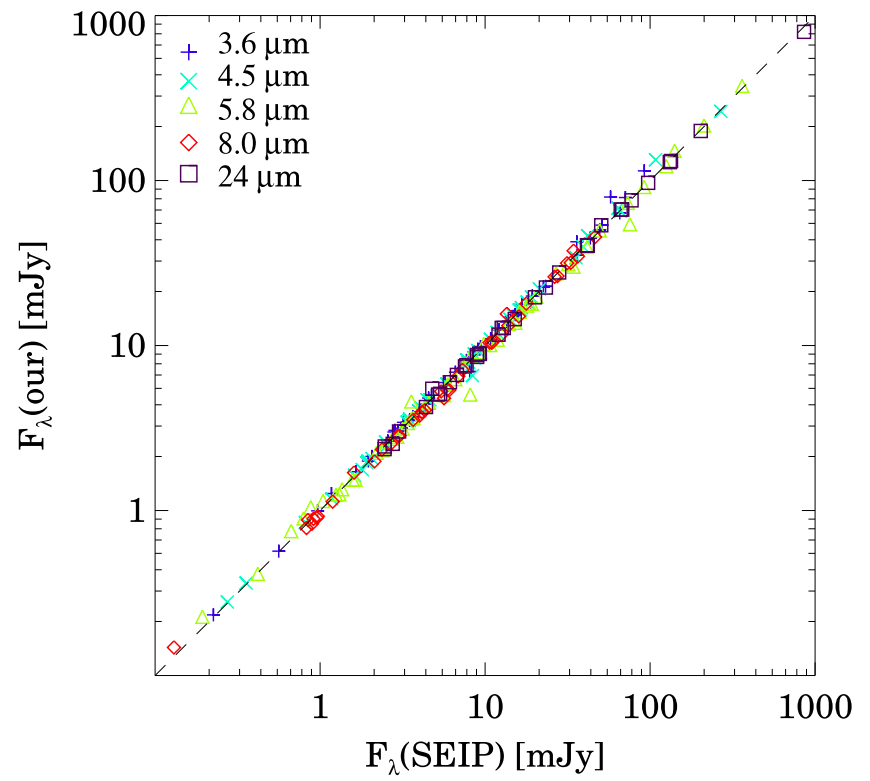

Figure 2. SEIP Source List fluxes of a sample of candidate YSOs plotted against those measured during the present work.

JHK bands, using the near-infrared camera Omega-Cass, mounted on the $3.5 \mathrm{~m}$ telescope at the Calar Alto Observatory, Spain. Our targets were IRAS $02224+7227$, the possible driving source of $\mathrm{HH} 487$, and the compact, partly embedded cluster RNO 7, centered on IRAS $02236+7224$. The results for IRAS 02224+7227 have been shown in Kun et al. (2014). Here we present the results for RNO 7.

Omega-Cass's detector was a Rockwell $1024 \times 1024$ pixel HAWAII array (HgCdTe detector $+\mathrm{Si}$ MOSFET nondestructive readout). The plate scale was 0 " 1 pixel $^{-1}$. RNO 7 was observed at four dithering positions around the nominal position of IRAS $02234+7224$, and the observations consisted of two dither cycles, and each cycle with $120 \mathrm{~s}(4 \times 30 \mathrm{~s}$ in $J$ and $H, 12 \times 10 \mathrm{~s}$ in $K$ ) spent at each position. Thus, the total on-source integration time of a cycle was $480 \mathrm{~s}$ in each filter. Double Correlated Read (Reset-Read-Read) was applied.

The data were reduced in IRAF. Following the flat-field correction and bad pixel removal, the sky frame for each cycle was obtained by taking the minimum of the images at different dithering positions. This sky frame was subtracted from each individual image of a given cycle. Then the frames from a single cycle were combined into a mosaic image, and aperture photometry was performed on the reduced images. The instrumental magnitudes were transformed into the $J H K_{\mathrm{s}}$ system by using the 2MASS magnitudes of 17 stars within the field of view. Then, in order to search for possible close visual companions, the point-spread functions (PSFs) of the images were determined, and the scaled PSFs of the stars were subtracted from the images.

\subsection{Supplementary Data}

To classify the evolutionary status of the color-selected candidate YSOs and obtain as complete a picture of the SFR and its YSO population as possible, we supplemented the Spitzer data with photometric data available in public databases. The databases included in our study are as follows.

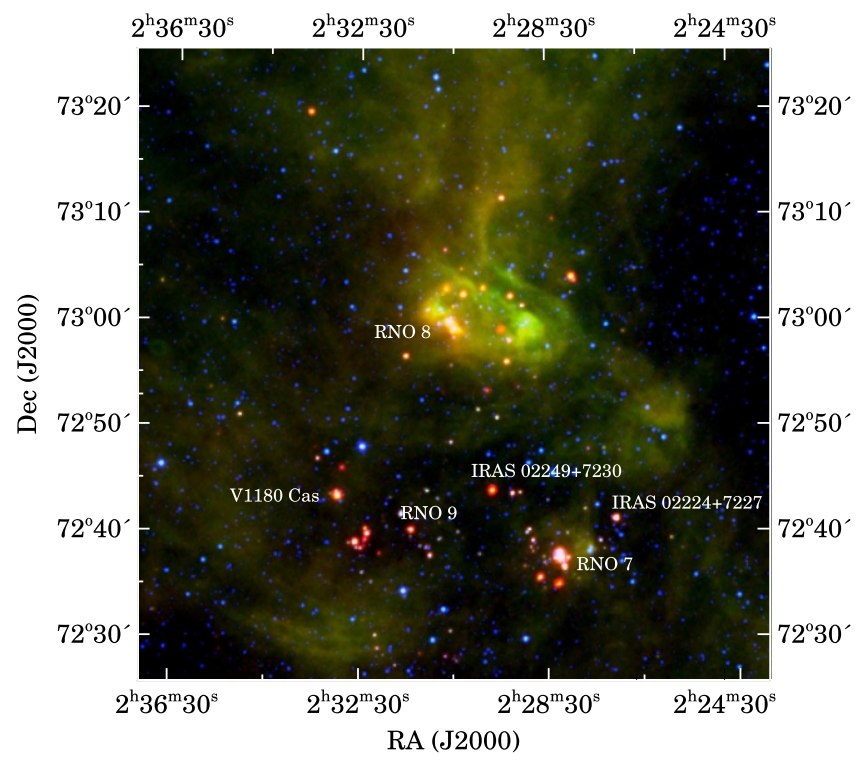

Figure 3. WISE $4.6 \mu \mathrm{m}$ (blue), $12 \mu \mathrm{m}$ (green), and $22 \mu \mathrm{m}$ (red) composite image of L1340. The size of the image is 1 square degree and is centered on R.A.(J2000) $=37^{\circ} .625$, decl.(J2000) $=+72^{\circ} .933$. Small groups of $22 \mu \mathrm{m}$ sources point to the three star-forming clumps of L1340. The extended $12 \mu \mathrm{m}$ emission (the Swan Nebula) is excited by the B- and A-type stars associated with L1340 B. The annotated objects are the most striking signposts of lowmass star formation: IRAS $02224+7227$ is an FUor-like star (Kun et al. 2014); IRAS $02249+7230$ is a protostellar source exciting HH 489; RNO 7, RNO 8, and RNO 9 are nebulous, partially embedded stellar groups (Cohen 1980); and V1180 Cas is an eruptive star (Kun et al. 2011).

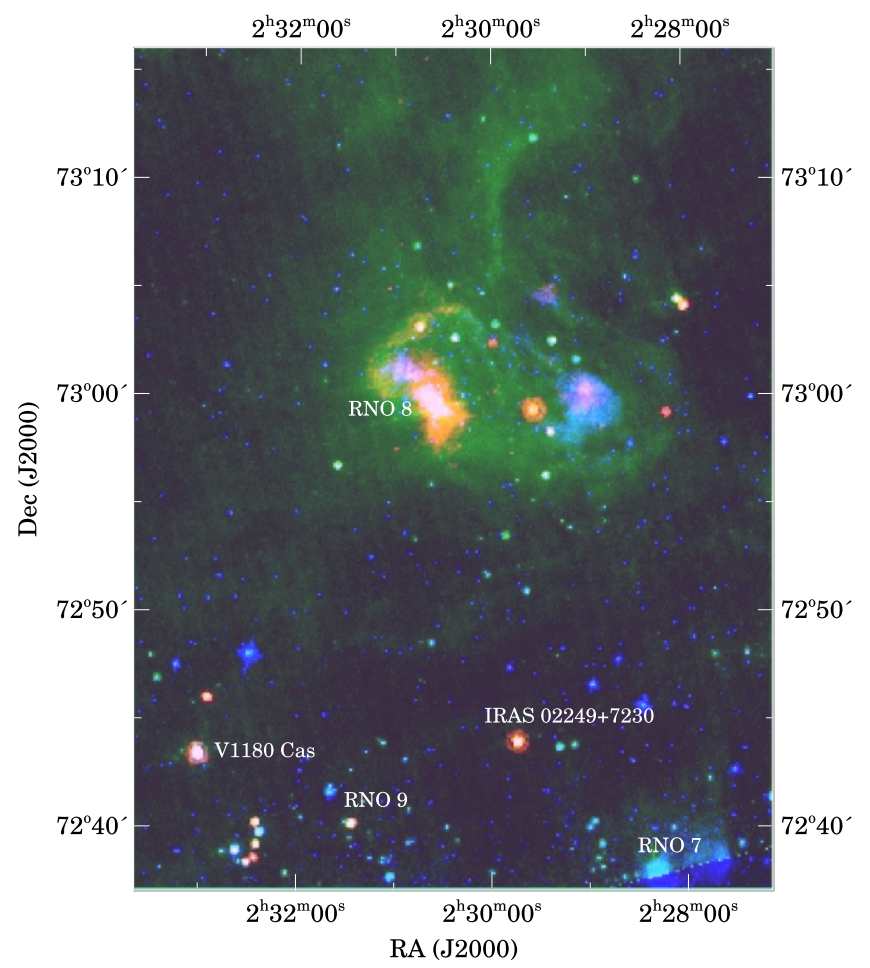

Figure 4. Three-color image of a $29^{\prime} \times 39^{\prime}$ size part of L1340, composed of Spitzer IRAC $5.8 \mu \mathrm{m}$ (blue), MIPS $24 \mu \mathrm{m}$ (green), and MIPS $70 \mu \mathrm{m}$ images. Part of L1340 A is outside the field of view of the MIPS images.

2MASS and AllWISE Data. The SEIP Source List contains WISE and 2MASS associations of the cataloged objects. WISE $22 \mu \mathrm{m}$ fluxes exist for 24 Spitzer-selected candidate 


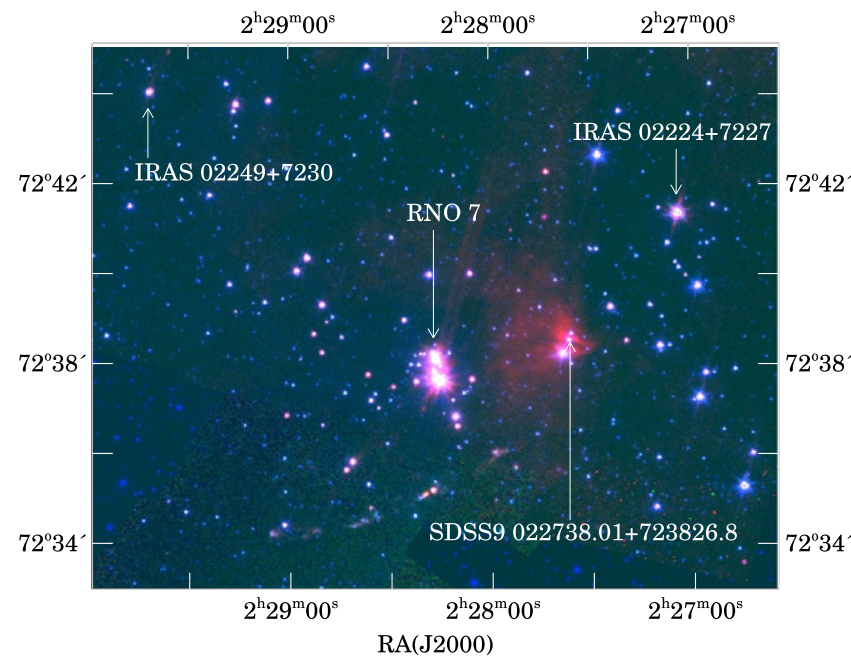

Figure 5. Three-color image of the cloud clump L1340 A, composed of Spitzer IRAC $3.6 \mu \mathrm{m}$ (blue), $5.8 \mu \mathrm{m}$ (green), and $8.0 \mu \mathrm{m}$ (red) images. The size of the image is about $15 ! 6 \times 12^{\prime}$. The arrows point to the positions of the FUor-like YSO IRAS 02224+7227, the A0-type star SDSS9 J022738.01+723826.8, associated with extended $8 \mu \mathrm{m}$ emission, the protostar IRAS $02249+7230$, and the RNO 7 cluster.

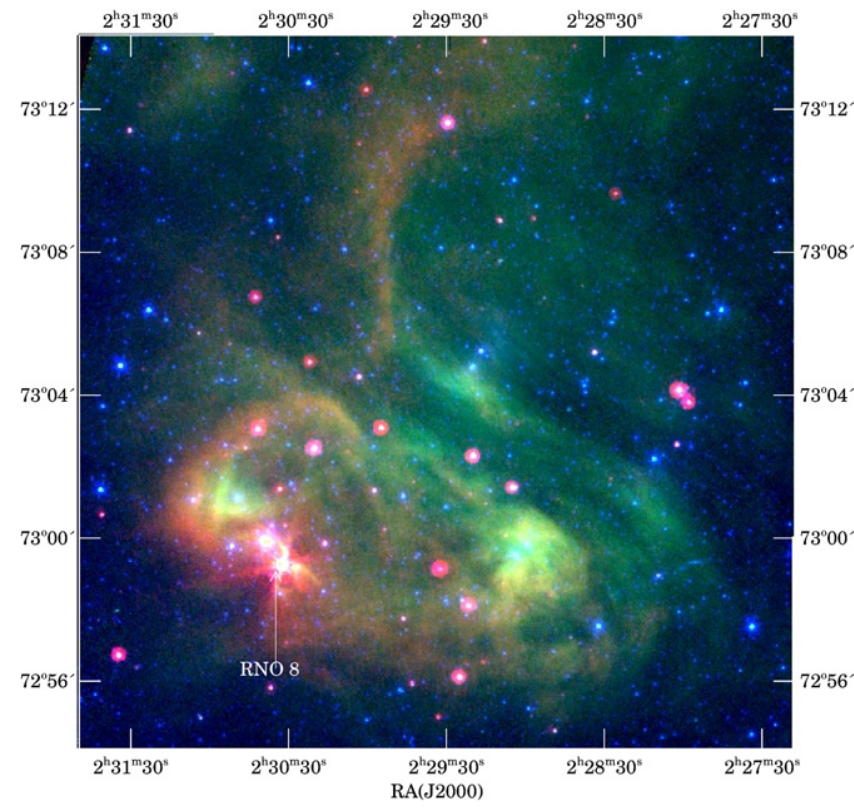

Figure 6. Three-color image of L1340 B, the Swan Nebula, composed of Spitzer IRAC $4.5 \mu \mathrm{m}$ (blue), $8.0 \mu \mathrm{m}$ (green), and MIPS $24 \mu \mathrm{m}$ (red) images.

YSOs outside of the area of the $24 \mu \mathrm{m}$ MIPS observations. We included these associations in the analysis, taking into account that, owing to the different angular resolutions, a few 2MASS/ WISE sources are associated with more than one IRAC source. Furthermore, we searched the AllWISE Source Catalog (Wright et al. 2010) for YSOs, using the color and flux criteria established by Koenig et al. (2012) and Koenig \& Leisawitz (2014). We identified eight new candidate YSOs, seven of which are located outside of the field of view of the Spitzer observations.

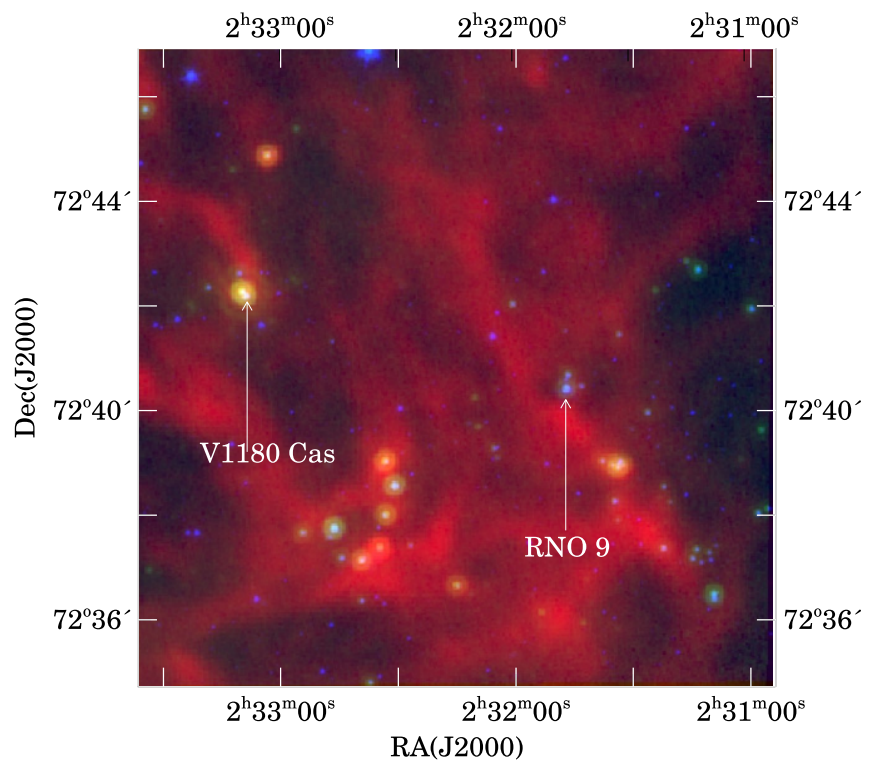

Figure 7. Three-color image of the clump L1340 C, composed of Spitzer IRAC $3.6 \mu \mathrm{m}$ (blue), MIPS $24 \mu \mathrm{m}$ (green), and Herschel SPIRE $250 \mu \mathrm{m}$ (red) images.

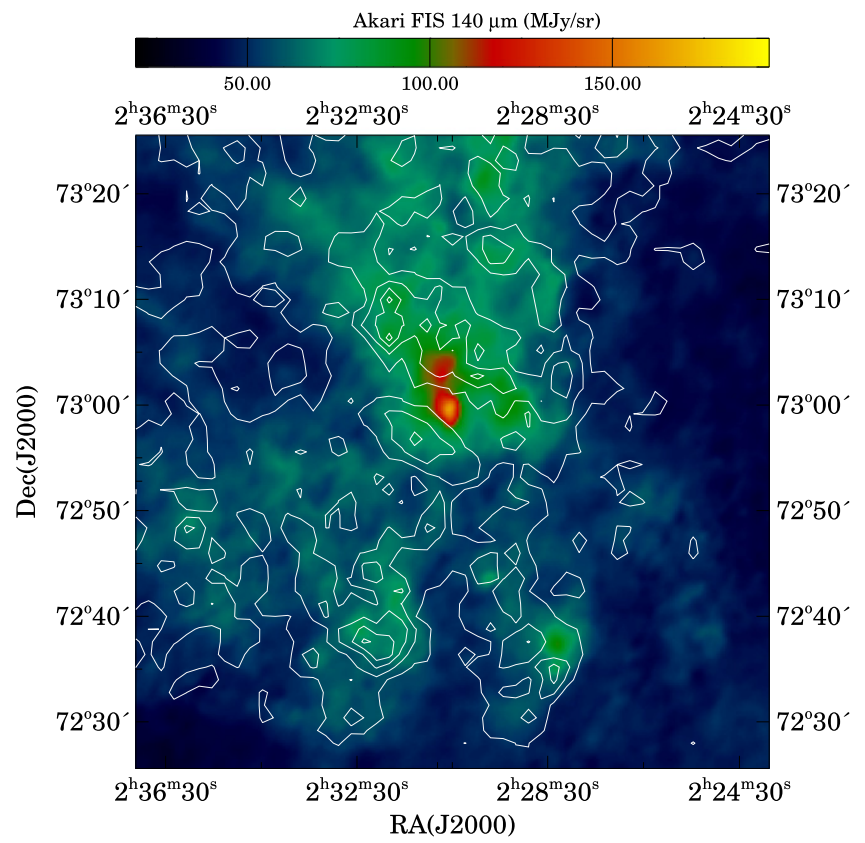

Figure 8. Akari Wide-L map of L1340 (colors) with the visual extinction (white solid contours; Kun et al. 2015) overplotted. Both the lowest contour and the increment for $A_{\mathrm{V}}$ are $1.0 \mathrm{mag}$. The center coordinates and size of the image are identical to those in Figure 3.

Akari FIS/IRC Data. Akari far-infrared all-sky survey images (Doi et al. 2015), tracing out the surface and temperature structure of the cold dust in the cloud region, are accessible at http://www.ir.isas.jaxa.jp/AKARI/Archive/ Images/FISMAP/. We identified counterparts of nine candidate YSOs in the Akari/FIS Bright Source Catalog (Yamamura et al. 2010), containing point sources detected at $65,90,140$, and $160 \mu \mathrm{m}$. 
Table 1

SEIP SSTSL2 Fluxes and Uncertainties of Class 0/I Sources in L1340

\begin{tabular}{|c|c|c|c|c|c|c|c|}
\hline No. & SSTSL2 & $\begin{array}{c}F_{3.6} \\
(\mathrm{mJy})\end{array}$ & $\begin{array}{c}F_{4.5} \\
(\mathrm{mJy})\end{array}$ & $\begin{array}{c}F_{5.8} \\
(\mathrm{mJy})\end{array}$ & $\begin{array}{c}F_{8.0} \\
(\mathrm{mJy})\end{array}$ & $\begin{array}{c}F_{24} \\
(\mathrm{mJy})\end{array}$ & $\begin{array}{l}F_{70}{ }^{\mathrm{a}} \\
(\mathrm{mJy})\end{array}$ \\
\hline 1 & $022756.91+730354.4$ & $1.915 \pm 0.005$ & $3.782 \pm 0.007$ & $5.412 \pm 0.016$ & $6.104 \pm 0.013$ & $73.620 \pm 0.164$ & $828.038 \pm 58.375$ \\
\hline 2 & $022800.65+730415.2$ & $14.510 \pm 0.014$ & $25.430 \pm 0.018$ & $40.200 \pm 0.044$ & $67.940 \pm 0.035$ & $289.700 \pm 0.263$ & $404.928 \pm 28.732$ \\
\hline 3 & $022808.60+725904.5$ & $1.774 \pm 0.005$ & $2.873 \pm 0.007$ & $2.982 \pm 0.013$ & $1.888 \pm 0.010$ & $1.775 \pm 0.132$ & $596.200 \pm 16.900$ \\
\hline 4 & $022818.51+723506.2$ & $1.586 \pm 0.004$ & $6.086 \pm 0.023$ & $8.608 \pm 0.020$ & $9.509 \pm 0.010$ & $\ldots$ & $\ldots$ \\
\hline 5 & $022820.81+723500.5$ & $0.251 \pm 0.001$ & $2.019 \pm 0.015$ & $4.189 \pm 0.014$ & $8.067 \pm 0.025$ & $\ldots$ & $\ldots$ \\
\hline 6 & $022825.07+730945.6$ & $0.150 \pm 0.002$ & $0.179 \pm 0.002$ & $0.197 \pm 0.007$ & $0.842 \pm 0.011$ & $22.590 \pm 0.135$ & $43.500 \pm 11.100$ \\
\hline 7 & $022842.57+723544.3$ & $11.560 \pm 0.010$ & $19.330 \pm 0.036$ & $27.290 \pm 0.034$ & $28.610 \pm 0.026$ & $\ldots$ & $\ldots$ \\
\hline 8 & $022844.40+723533.5$ & $3.473 \pm 0.005$ & $4.359 \pm 0.024$ & $7.668 \pm 0.019$ & $7.259 \pm 0.018$ & $\ldots$ & $\ldots$ \\
\hline 9 & $022844.71+730308.5$ & $0.110 \pm 0.002$ & $0.135 \pm 0.002$ & $0.155 \pm 0.007$ & $0.284 \pm 0.012$ & $0.935 \pm 0.121$ & $\cdots$ \\
\hline 10 & $022849.44+723731.6$ & $0.035 \pm 0.001$ & $0.079 \pm 0.002$ & $0.105 \pm 0.005$ & $<0.023$ & $<0.491$ & $\ldots$ \\
\hline 11 & $022855.69+731333.1$ & $0.039 \pm 0.001$ & $0.063 \pm 0.002$ & $0.075 \pm 0.006$ & $0.083 \pm 0.008$ & $0.858 \pm 0.132$ & $\ldots$ \\
\hline 12 & $022856.61+730903.2$ & $0.108 \pm 0.002$ & $0.146 \pm 0.002$ & $0.212 \pm 0.006$ & $0.337 \pm 0.007$ & $2.936 \pm 0.135$ & $\cdots$ \\
\hline 13 & $022906.09+730210.5$ & $0.022 \pm 0.001$ & $0.030 \pm 0.002$ & $0.039 \pm 0.004$ & $0.063 \pm 0.008$ & $0.657 \pm 0.124$ & $\ldots$ \\
\hline 14 & $022914.62+730102.8$ & $0.063 \pm 0.001$ & $0.081 \pm 0.002$ & $\ldots$ & $0.381 \pm 0.007$ & $1.270 \pm 0.137$ & $\ldots$ \\
\hline 15 & $022918.25+724754.0$ & $0.081 \pm 0.001$ & $0.097 \pm 0.002$ & $0.087 \pm 0.006$ & $0.074 \pm 0.008$ & $3.509 \pm 0.127$ & $64.600 \pm 9.80$ \\
\hline 16 & $022931.98+725912.4$ & $0.959 \pm 0.003$ & $1.755 \pm 0.005$ & $1.699 \pm 0.010$ & $1.159 \pm 0.009$ & $211.700 \pm 0.218$ & $1949.471 \pm 136.77$ \\
\hline 17 & $022932.31+725503.2^{\mathrm{b}}$ & $0.226 \pm 0.002$ & $0.274 \pm 0.002$ & $0.419 \pm 0.006$ & $0.956 \pm 0.009$ & $5.341 \pm 0.134$ & $\ldots$ \\
\hline 18 & $022943.01+724359.6$ & $\ldots$ & $2.118 \pm 0.005$ & $3.482 \pm 0.005$ & $6.541 \pm 0.015$ & $<0.659$ & $\ldots$ \\
\hline 19 & $022943.64+724358.6$ & $2.316 \pm 0.004$ & $7.394 \pm 0.012$ & $13.270 \pm 0.024$ & $19.350 \pm 0.021$ & $375.200 \pm 0.250$ & $2496.581 \pm 175.06$ \\
\hline 20 & $022949.62+725326.1$ & $0.238 \pm 0.002$ & $0.428 \pm 0.003$ & $0.622 \pm 0.007$ & $1.286 \pm 0.010$ & $53.000 \pm 0.153$ & $103.044 \pm 7.602$ \\
\hline 21 & $022955.10+730309.1$ & $1.703 \pm 0.005$ & $3.281 \pm 0.008$ & $6.332 \pm 0.018$ & $14.200 \pm 0.018$ & $58.580 \pm 0.165$ & $51.500 \pm 21.000$ \\
\hline 22 & $022956.90+730217.0^{c}$ & $\ldots$ & $\ldots$ & $\ldots$ & $\ldots$ & $\ldots$ & $262.0 \pm 36.6$ \\
\hline 23 & $023022.78+730459.0$ & $1.137 \pm 0.004$ & $1.790 \pm 0.006$ & $2.709 \pm 0.012$ & $3.747 \pm 0.013$ & $24.220 \pm 0.155$ & $69.852 \pm 5.871$ \\
\hline 24 & $023030.42+725706.7$ & $0.084 \pm 0.001$ & $0.108 \pm 0.002$ & $0.156 \pm 0.005$ & $0.293 \pm 0.008$ & $2.228 \pm 0.126$ & $\ldots$ \\
\hline 25 & $023032.44+725918.0^{\mathrm{b}}$ & $79.412 \pm 3.192$ & $115.791 \pm 4.498^{\mathrm{a}}$ & $215.566 \pm 7.104$ & $528.239 \pm 18.993^{\mathrm{a}}$ & $2069.692 \pm 82.791$ & $1876.886 \pm 132.659$ \\
\hline 26 & $023035.51+730828.2$ & $0.227 \pm 0.002$ & $0.466 \pm 0.003$ & $0.753 \pm 0.008$ & $1.187 \pm 0.010$ & $3.066 \pm 0.113$ & $\ldots$ \\
\hline 27 & $023042.36+730305.1$ & $4.773 \pm 0.009$ & $13.510 \pm 0.016$ & $24.490 \pm 0.034$ & $28.800 \pm 0.024$ & $117.100 \pm 0.180$ & $484.031 \pm 34.168$ \\
\hline 28 & $023127.34+724012.9$ & $2.280 \pm 0.072$ & $5.119 \pm 0.353$ & $10.224 \pm 0.313$ & $15.729 \pm 0.518$ & $101.771 \pm 4.074$ & $663.806 \pm 46.801$ \\
\hline 29 & $023134.23+725829.1$ & $0.057 \pm 0.001$ & $0.090 \pm 0.001$ & $0.119 \pm 0.005$ & $0.075 \pm 0.007$ & $0.782 \pm 0.119$ & $\ldots$ \\
\hline 30 & $023142.50+725740.4$ & $0.049 \pm 0.001$ & $0.069 \pm 0.002$ & $0.076 \pm 0.005$ & $0.355 \pm 0.010$ & $0.808 \pm 0.123$ & $\cdots$ \\
\hline 31 & $023146.58+723729.4$ & $\ldots$ & $\ldots$ & $0.256 \pm 0.006$ & $\ldots$ & $3.952 \pm 0.128$ & $\cdots$ \\
\hline 32 & $023203.42+724131.7$ & $0.062 \pm 0.001$ & $0.177 \pm 0.002$ & $0.417 \pm 0.008$ & $0.930 \pm 0.012$ & $3.437 \pm 0.112$ & $\ldots$ \\
\hline 33 & $023207.96+723759.3$ & $0.784 \pm 0.003$ & $\ldots$ & $3.255 \pm 0.013$ & $\ldots$ & $26.570 \pm 0.146$ & $162.799 \pm 11.629$ \\
\hline 34 & $023225.98+724020.1$ & $\ldots$ & $\ldots$ & $9.834 \pm 0.021$ & $\ldots$ & $79.120 \pm 0.163$ & $626.957 \pm 44.163$ \\
\hline 35 & $023226.35+723919.4$ & $2.839 \pm 0.005$ & $\cdots$ & $1.752 \pm 0.009$ & $\cdots$ & $72.926 \pm 1.888$ & $375.855 \pm 27.889$ \\
\hline 36 & $023227.64+723841.4^{\mathrm{d}}$ & $0.693 \pm 0.022$ & $\ldots$ & $0.847 \pm 0.033$ & $\cdots$ & $17.613 \pm 0.715$ & $378.511 \pm 27.082$ \\
\hline 37 & $023232.00+723827.5$ & $4.784 \pm 0.006$ & $\ldots$ & $13.880 \pm 0.025$ & $\ldots$ & $57.910 \pm 0.124$ & $343.451 \pm 24.445$ \\
\hline 38 & $023237.90+723940.7$ & $0.094 \pm 0.001$ & $\ldots$ & $0.543 \pm 0.007$ & $\ldots$ & $<0.361$ & $\ldots$ \\
\hline 39 & $023248.83+724635.4$ & $0.305 \pm 0.002$ & $0.251 \pm 0.002$ & $0.226 \pm 0.007$ & $2.142 \pm 0.009$ & $3.936 \pm 0.138$ & $\ldots$ \\
\hline 40 & $023256.14+724605.3$ & $1.338 \pm 0.003$ & $1.697 \pm 0.004$ & $1.639 \pm 0.010$ & $1.428 \pm 0.008$ & $57.310 \pm 0.147$ & $847.044 \pm 59.580$ \\
\hline 41 & $023302.41+724331.2$ & $5.987 \pm 0.007$ & $14.790 \pm 0.012$ & $27.930 \pm 0.035$ & $57.890 \pm 0.033$ & $530.100 \pm 0.265$ & $2281.543 \pm 160.168$ \\
\hline 42 & $023330.92+724800.3$ & $0.162 \pm 0.002$ & $0.248 \pm 0.002$ & $0.246 \pm 0.007$ & $0.297 \pm 0.009$ & $11.950 \pm 0.128$ & $\ldots$ \\
\hline 43 & $023340.83+731950.8$ & $6.178 \pm 0.007$ & $11.280 \pm 0.010$ & $16.950 \pm 0.029$ & $24.350 \pm 0.031$ & $\ldots$ & $\cdots$ \\
\hline 44 & $023432.66+724057.2$ & $0.243 \pm 0.002$ & $\ldots$ & $0.636 \pm 0.007$ & $\ldots$ & $3.180 \pm 0.117$ & $\cdots$ \\
\hline 45 & $023532.06+724922.6$ & $0.079 \pm 0.001$ & $0.098 \pm 0.002$ & $0.101 \pm 0.006$ & $0.138 \pm 0.008$ & $2.856 \pm 0.133$ & $\cdots$ \\
\hline
\end{tabular}

Notes.

${ }^{\mathrm{a}}$ This flux results from our measurement.

${ }^{\mathrm{b}} \mathrm{H} \alpha$ emission star.

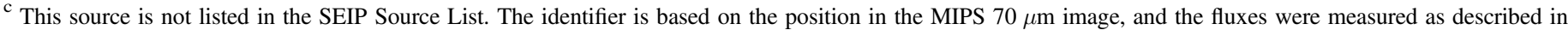
Section 2.1 .

d This source is not listed in the SEIP Source List. The identifier is based on the position in the SEIP Super Mosaic images of the region.

(This table is available in machine-readable form.)

Submillimeter Data. Part of the molecular clump L1340 B was observed at 450 and $850 \mu \mathrm{m}$ with the Submillimetre Common User Bolometer Array (SCUBA) on the James Clerk Maxwell Telescope. The outlines of the mapped area are shown in Figure 6 of Paper III. The $850 \mu \mathrm{m}$ image and positions, sizes, and fluxes/upper limits of nine submillimeter sources can be found in the SCUBA Legacy Catalogs (Di Francesco et al. 2008), at http://www3.cadc-ccda.hia-iha.nrc-cnrc.gc.ca/ community/scubalegacy/. Four of them coincide in position with Spitzer sources.

Herschel Data for L1340 C. The Planck Galactic cold clump PGCC G130.38+11.26, associated with L1340C, was included in the detailed Herschel study of cold clumps by Juvela et al. (2012). Far-infrared images, observed by the 
Table 2

SEIP SSTSL2 Fluxes and Uncertainties of Flat SED Sources in L1340

\begin{tabular}{|c|c|c|c|c|c|c|c|}
\hline No. & SSTSL2 & $\begin{array}{c}F_{3.6} \\
(\mathrm{mJy})\end{array}$ & $\begin{array}{c}F_{4.5} \\
(\mathrm{mJy})\end{array}$ & $\begin{array}{c}F_{5.8} \\
(\mathrm{mJy})\end{array}$ & $\begin{array}{c}F_{8.0} \\
(\mathrm{mJy})\end{array}$ & $\begin{array}{c}F_{24} \\
(\mathrm{mJy})\end{array}$ & $\begin{array}{l}F_{70}{ }^{\mathrm{a}, \mathrm{b}} \\
(\mathrm{mJy})\end{array}$ \\
\hline 1 & $022754.00+723535.5$ & $1.744 \pm 0.004$ & $1.766 \pm 0.020$ & $2.957 \pm 0.012$ & $0.245 \pm 0.018$ & $\cdots$ & $\cdots$ \\
\hline 2 & $022759.92+723556.4^{b}$ & $0.523 \pm 0.026$ & $0.859 \pm 0.060$ & $0.980 \pm 0.032$ & $\ldots$ & $\cdots$ & \\
\hline 3 & $022811.32+723631.5$ & $4.896 \pm 0.086$ & $6.002 \pm 0.011$ & $6.895 \pm 0.011$ & $7.715 \pm 0.014$ & $\cdots$ & $\cdots$ \\
\hline 4 & $022816.62+723732.6$ & $219.545 \pm 28.270$ & $268.500 \pm 0.135$ & $361.400 \pm 0.124$ & $374.611 \pm 11.495^{\mathrm{a}}$ & $\ldots$ & $\ldots$ \\
\hline 5 & $022817.85+723800.9$ & $92.280 \pm 0.027$ & $108.000 \pm 0.056$ & $140.700 \pm 0.078$ & $219.498 \pm 6.839^{\mathrm{a}}$ & $857.300 \pm 0.612$ & $\ldots$ \\
\hline 6 & $022818.51+723734.6$ & $\cdots$ & $9.617 \pm 0.021$ & $8.287 \pm 0.019$ & $9.695 \pm 0.015$ & $\cdots$ & $\cdots$ \\
\hline 7 & $022838.02+723740.6$ & $2.805 \pm 0.005$ & $2.963 \pm 0.006$ & $3.217 \pm 0.012$ & $5.370 \pm 0.012$ & $12.010 \pm 0.516$ & $\cdots$ \\
\hline 8 & $022850.36+723851.2$ & $0.822 \pm 0.003$ & $0.883 \pm 0.004$ & $0.897 \pm 0.008$ & $1.218 \pm 0.009$ & $4.005 \pm 0.180$ & $\cdots$ \\
\hline 9 & $022851.83+723810.2$ & $2.934 \pm 0.005$ & $3.027 \pm 0.007$ & $2.980 \pm 0.012$ & $3.900 \pm 0.012$ & $16.730 \pm 0.215$ & $\cdots$ \\
\hline 10 & $022858.15+723801.4$ & $0.461 \pm 0.002$ & $0.607 \pm 0.003$ & $0.627 \pm 0.007$ & $0.551 \pm 0.009$ & $4.872 \pm 0.185$ & $\cdots$ \\
\hline 11 & $022907.88+724347.2$ & $3.938 \pm 0.006$ & $5.972 \pm 0.010$ & $8.489 \pm 0.020$ & $11.680 \pm 0.017$ & $30.940 \pm 0.142$ & $66.994 \pm 5.117$ \\
\hline 12 & $022917.57+723904.7$ & $0.735 \pm 0.003$ & $0.803 \pm 0.005$ & $0.818 \pm 0.008$ & $0.853 \pm 0.010$ & $\ldots$ & $\cdots$ \\
\hline 13 & $022919.60+730223.5$ & $15.980 \pm 0.014$ & $21.360 \pm 0.018$ & $26.930 \pm 0.036$ & $46.410 \pm 0.029$ & $131.700 \pm 0.180$ & $181.009 \pm 20.0$ \\
\hline 14 & $022920.70+730119.0$ & $0.137 \pm 0.002$ & $0.171 \pm 0.002$ & $0.156 \pm 0.005$ & $0.169 \pm 0.007$ & $1.491 \pm 0.128$ & $\cdots$ \\
\hline 15 & $022950.37+724441.4$ & $0.069 \pm 0.001$ & $0.092 \pm 0.002$ & $0.163 \pm 0.005$ & $0.341 \pm 0.009$ & $0.877 \pm 0.120$ & $\cdots$ \\
\hline 16 & $023020.61+730233.7$ & $30.700 \pm 0.023$ & $35.390 \pm 0.029$ & $47.510 \pm 0.048$ & $61.830 \pm 0.034$ & $174.500 \pm 0.212$ & $162.696 \pm 11.950$ \\
\hline 17 & $022920.70+730119.0$ & $0.137 \pm 0.002$ & $0.171 \pm 0.002$ & $0.156 \pm 0.005$ & $0.169 \pm 0.007$ & $1.491 \pm 0.128$ & \\
\hline 18 & $023033.71+730125.1$ & $0.957 \pm 0.004$ & $1.813 \pm 0.005$ & $2.940 \pm 0.013$ & $4.755 \pm 0.012$ & $11.630 \pm 0.134$ & $\cdots$ \\
\hline 19 & $023049.81+731049.2$ & $0.059 \pm 0.002$ & $0.084 \pm 0.002$ & $0.128 \pm 0.006$ & $0.255 \pm 0.007$ & $0.581 \pm 0.100$ & $\cdots$ \\
\hline 20 & $023053.25+730528.5$ & $0.087 \pm 0.001$ & $0.109 \pm 0.002$ & $0.091 \pm 0.005$ & $0.366 \pm 0.009$ & $0.871 \pm 0.123$ & $\cdots$ \\
\hline 21 & $023114.12+723933.3$ & $0.480 \pm 0.002$ & $0.462 \pm 0.004$ & $0.482 \pm 0.007$ & $0.568 \pm 0.019$ & $2.432 \pm 0.125$ & $\cdots$ \\
\hline 22 & $023127.45+723912.8$ & $0.488 \pm 0.002$ & $\ldots$ & $0.592 \pm 0.007$ & $\ldots$ & $2.234 \pm 0.132$ & $\cdots$ \\
\hline 23 & $023127.52+725621.5$ & $0.133 \pm 0.001$ & $0.157 \pm 0.002$ & $0.150 \pm 0.005$ & $0.447 \pm 0.009$ & $1.513 \pm 0.123$ & $\cdots$ \\
\hline 24 & $023134.62+725642.0$ & $16.900 \pm 0.012$ & $18.840 \pm 0.013$ & $19.710 \pm 0.031$ & $28.240 \pm 0.032$ & $82.320 \pm 0.177$ & $180.167 \pm 12.921$ \\
\hline 25 & $023247.15+723858.8$ & $2.079 \pm 0.004$ & $\cdots$ & $2.887 \pm 0.005$ & $\cdots$ & $17.390 \pm 0.126$ & $\cdots$ \\
\hline 26 & $023254.71+724257.9$ & $0.077 \pm 0.001$ & $0.132 \pm 0.002$ & $0.211 \pm 0.005$ & $0.378 \pm 0.009$ & $1.131 \pm 0.104$ & $\cdots$ \\
\hline 27 & $023301.52+724326.7$ & $43.430 \pm 0.018$ & $63.980 \pm 0.025$ & $91.940 \pm 0.064$ & $\cdots$ & $<0.325$ & $\cdots$ \\
\hline
\end{tabular}

Notes.

${ }^{a}$ This flux results from our measurements.

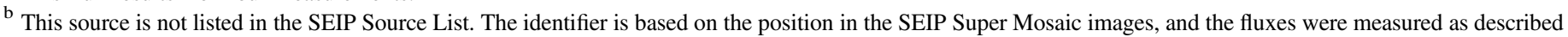
in Section 2.1 .

(This table is available in machine-readable form.)

Table 3

SEIP SSTSL2 Fluxes and Uncertainties of of Class II Sources

\begin{tabular}{|c|c|c|c|c|c|c|}
\hline SSTSL2 & $\begin{array}{c}F_{3.6} \\
(\mathrm{mJy})\end{array}$ & $\begin{array}{c}F_{4.5} \\
(\mathrm{mJy})\end{array}$ & $\begin{array}{c}F_{5.8} \\
(\mathrm{mJy})\end{array}$ & $\begin{array}{c}F_{8.0} \\
(\mathrm{mJy})\end{array}$ & $\begin{array}{c}F_{24} \\
(\mathrm{mJy})\end{array}$ & $\begin{array}{c}F_{70} \\
(\mathrm{mJy})\end{array}$ \\
\hline $022638.02+730457.5^{\mathrm{a}}$ & $3.517 \pm 0.147$ & $3.965 \pm 0.009$ & $4.335 \pm 0.015$ & $5.878 \pm 0.015$ & $7.710 \pm 0.116$ & $36.198 \pm 3.178$ \\
\hline $022654.73+724040.8$ & $1.552 \pm 0.004$ & $1.012 \pm 0.004$ & $0.527 \pm 0.007$ & $0.461 \pm 0.010$ & $4.301 \pm 0.179$ & $\ldots$ \\
\hline $022659.03+725716.0$ & $1.477 \pm 0.005$ & $1.156 \pm 0.003$ & $0.921 \pm 0.006$ & $0.739 \pm 0.009$ & $5.040 \pm 0.115$ & $\ldots$ \\
\hline $022659.08+724016.6^{\mathrm{a}}$ & $0.966 \pm 0.003$ & $0.815 \pm 0.004$ & $0.667 \pm 0.007$ & $0.893 \pm 0.009$ & $1.221 \pm 0.200$ & $\ldots$ \\
\hline $022659.35+725714.2$ & $1.581 \pm 0.005$ & $1.204 \pm 0.003$ & $0.981 \pm 0.006$ & $1.098 \pm 0.009$ & $9.337 \pm 0.117$ & $\ldots$ \\
\hline $022700.34+724743.8^{\mathrm{a}}$ & $2.571 \pm 0.005$ & $1.920 \pm 0.006$ & $1.573 \pm 0.009$ & $2.136 \pm 0.010$ & $8.947 \pm 0.126$ & $\ldots$ \\
\hline $022702.11+724329.0^{\mathrm{a}}$ & $2.061 \pm 0.004$ & $1.617 \pm 0.006$ & $1.043 \pm 0.008$ & $0.968 \pm 0.011$ & $2.460 \pm 0.123$ & $\ldots$ \\
\hline $022703.17+723952.9^{\mathrm{a}}$ & $1.172 \pm 0.003$ & $1.177 \pm 0.005$ & $0.794 \pm 0.007$ & $1.192 \pm 0.009$ & $2.206 \pm 0.179$ & $\ldots$ \\
\hline $022705.53+724116.7$ & $169.100 \pm 5.464$ & $131.753 \pm 5.460^{\mathrm{b}}$ & $125.8 \pm 4.288$ & $147.395 \pm 4.713^{\mathrm{b}}$ & $197.200 \pm 0.303$ & $\ldots$ \\
\hline $022706.29+724011.1^{\mathrm{a}}$ & $1.918 \pm 0.070^{\mathrm{b}}$ & $1.344 \pm 0.044^{\mathrm{b}}$ & $0.878 \pm 0.008$ & $0.912 \pm 0.009$ & $2.466 \pm 0.181$ & $\ldots$ \\
\hline
\end{tabular}

Notes.

${ }^{\mathrm{a}} \mathrm{H} \alpha$ emission star, described in detail in Paper III.

b Our measurement.

(This table is available in its entirety in machine-readable form.)

PACS instrument at 100 and $160 \mu \mathrm{m}$, as well as 250,350 , and $500 \mu \mathrm{m}$ images observed by the SPIRE instrument, are available in the Herschel Science Archive (http://www. cosmos.esa.int/web/herschel/science-archive). We found far- infrared counterparts of 20 color-selected Spitzer sources in the PACS 100 and $160 \mu \mathrm{m}$ images. We measured the fluxes of the sources on the level2.5 JScanam images, downloaded from the Herschel Science Archive (Galactic Cold Cores: A 
Table 4

Herschel PACS Point Sources Associated with Candidate Spitzer YSOs in L1340 C

\begin{tabular}{|c|c|c|c|c|c|c|c|}
\hline No. & SSTSL2 & $\begin{array}{l}\text { Fitted R.A. } \\
\text { (degree) }\end{array}$ & $\begin{array}{l}\text { Fitted Decl. } \\
\quad(\text { degree })\end{array}$ & $\begin{array}{l}F_{100} \\
(\mathrm{mJy})\end{array}$ & $\begin{array}{l}d F_{100} \\
(\mathrm{mJy})\end{array}$ & $\begin{array}{l}F_{160} \\
(\mathrm{mJy})\end{array}$ & $\begin{array}{l}d F_{160} \\
(\mathrm{mJy})\end{array}$ \\
\hline 1 & $022907.88+724347.2$ & 37.28481 & 72.73014 & 349.372 & $\ldots$ & 1073.854 & $\ldots$ \\
\hline 2 & $022943.64+724358.6$ & 37.43316 & 72.73337 & 9899.194 & $\cdots$ & 11999.27 & $\ldots$ \\
\hline 3 & $023114.12+723933.3$ & 37.82169 & 72.66068 & 34.923 & 353.948 & $\ldots$ & $\cdots$ \\
\hline 4 & $023127.45+723912.8$ & 37.87831 & 72.65379 & 13.773 & 101.163 & 0474.558 & 38.295 \\
\hline 5 & $023127.34+724013.0$ & 37.86170 & 72.67144 & 918.261 & 124.522 & 1652.396 & 155.898 \\
\hline 6 & $023146.58+723729.4$ & 37.93610 & 72.62243 & 16.760 & 57.825 & 0342.640 & 66.379 \\
\hline 7 & $023203.42+724131.7$ & 38.01794 & 72.68809 & 0.424 & 182.745 & 0101.090 & 62.318 \\
\hline 8 & $023207.96+723759.3$ & 38.03459 & 72.63372 & 258.719 & 205.061 & 0500.088 & 85.020 \\
\hline 9 & $023225.98+724020.1$ & 38.11119 & 72.67221 & 879.202 & 47.523 & 1712.686 & 87.426 \\
\hline 10 & $023226.35+723919.4$ & 38.10945 & 72.65580 & 488.341 & 196.238 & 2403.526 & 376.658 \\
\hline 11 & $023227.64+723841.4$ & 38.11722 & 72.64574 & 1118.237 & 187.990 & 2461.969 & 379.767 \\
\hline 12 & $023232.00+723827.5$ & 38.13490 & 72.64143 & 639.157 & 122.241 & 2462.245 & 379.627 \\
\hline 13 & $023237.90+723940.7$ & 38.15584 & 72.66280 & 22.780 & 114.644 & 264.833 & 156.138 \\
\hline 14 & $023247.15+723858.8$ & 38.19769 & 72.65025 & 37.372 & 194.085 & 175.280 & 226.861 \\
\hline 15 & $023248.83+724635.4$ & 38.20506 & 72.77680 & 75.233 & 51.971 & $\ldots$ & $\ldots$ \\
\hline 16 & $023254.71+724257.9$ & 38.22334 & 72.71396 & 7.888 & 142.752 & $\ldots$ & $\ldots$ \\
\hline 17 & $023256.14+724605.3$ & 38.23558 & 72.76854 & 1798.065 & 105.467 & 2059.933 & 84.941 \\
\hline 18 & $023302.41+724331.2$ & 38.26159 & 72.72580 & 2621.677 & 155.127 & 2801.787 & 100.030 \\
\hline 19 & $023331.04+724800.8$ & 38.38035 & 72.80073 & 119.108 & 168.020 & 470.005 & 69.517 \\
\hline 20 & $023432.66+724057.2$ & 38.63831 & 72.68040 & 23.361 & 173.174 & $\ldots$ & $\ldots$ \\
\hline
\end{tabular}

Herschel survey of the source populations revealed by Planck; PI: M. Juvela). The photometry was performed using the L3_multiplePointSourceAperturePhotometry . py, supplied in HIPE 14.0 RC4 (Herschel Interactive Processing Environment; Ott 2010). We used 6" and 10" apertures at 100 and $160 \mu \mathrm{m}$, respectively, with an annulus between $35^{\prime \prime}$ and $45^{\prime \prime}$ for determining the background. The aperture correction were calculated using the values given in Balog et al. (2014). The initial positions of the sources were taken from the SEIP Source List and were refined using a twodimensional Gaussian during the photometry.

SDSS Data. SDSS ugriz magnitudes are available for each star brighter than some 25 mag in each band within the whole area of L1340 (see Paper III). We searched for counterparts of our candidate YSOs in the SDSS Data Release 9 (Ahn et al. 2012) within $1^{\prime \prime}$ of the SEIP Source List position. We transformed the SDSS magnitudes of the optical counterparts into the Johnson-Cousins $U B V R_{\mathrm{C}} I_{\mathrm{C}}$ system, using the equations given in Ivezić et al. (2007; for $B V R_{\mathrm{C}} I_{\mathrm{C}}$ ) and Jordi et al. (2006; for $U$ ). We found optical counterparts for 149 of the 155 Class II Spitzer sources, and for 8 of the 26 flat spectral energy distribution (SED) sources (see Section 4.1).

\section{INFRARED APPEARANCE OF L1340: THE SWAN NEBULA}

The extended infrared emission reveals the surface distribution of various components of the cloud. Cold $\left(T_{\text {kin }} \sim\right.$ $10-20 \mathrm{~K})$, big $(r \gtrsim 0.1 \mu \mathrm{m})$ dust grains radiate in the farinfrared, whereas extended mid-infrared emission traces out very small grains and excited $\mathrm{PAH}$ molecules. Heating and shocks from embedded YSOs also appear in the infrared images of a molecular cloud.

Figure 3 shows a three-color view of L1340, composed of the WISE $4.6 \mu \mathrm{m}$ (blue), $12 \mu \mathrm{m}$ (green), and $22 \mu \mathrm{m}$ (red) images. Striking features of this image are the bright, extended $12 \mu \mathrm{m}$ radiation, indicative of $\mathrm{PAH}$ emission excited by $\mathrm{B}-$ and
A-type stars, and small groups of $22 \mu \mathrm{m}$ sources, associated with the three cloud clumps. The shape of the brightest part of the diffuse $12 \mu \mathrm{m}$ emission, located slightly northwest of the image center and associated with the clump L1340 B, suggests the Swan Nebula label.

Figure 4 is a composite of the $5.8 \mu \mathrm{m}$ IRAC (blue), $24 \mu \mathrm{m}$ MIPS (green), and $70 \mu \mathrm{m}$ MIPS (red) images. The image reveals an extended $70 \mu \mathrm{m}$ structure associated with RNO 8, diffuse $24 \mu \mathrm{m}$ emission that delineates the Swan Nebula, a bluish $(5.8 \mu \mathrm{m})$ glowing around the B-type stars, and a variety of far-infrared point sources.

To reveal further details of the diffuse infrared emission of L1340, we present three-color images of the clumps L1340 A, L1340 B, and L1340 C in Figures 5-7, respectively. Figure 5 is composed of IRAC $3.6 \mu \mathrm{m}$ (blue), $4.5 \mu \mathrm{m}$ (green), and $8.0 \mu \mathrm{m}$ (red) Super Mosaic images of L1340 A (much of this clump is outside of the MIPS images). Conspicuous features of the image are a diffuse $8 \mu \mathrm{m}$ emission around the A0-type star SDSS9 $022738.01+723826.8$ (Paper III), the nebulous RNO 7 cluster, and $\mathrm{HH} 488$, stretching from NW toward SE near the southern boundary of the image. Figure 6 is composed of the $3.6 \mu \mathrm{m}$ (blue), $8.0 \mu \mathrm{m}$ (green), and $24 \mu \mathrm{m}$ (red) images of the most massive clump L1340 B. The wispy structure of the Swan Nebula, suggesting a swirling gas cloud, becomes apparent in this image. A bow-shock-like feature can be seen around the star SDSS9 J023049.80+730110.2, demonstrating supersonic motion of the gas with respect to the A2-type, young intermediate-mass star (Paper III). The extended infrared emission from the smallest clump, L1340 C, shows up in the Herschel images, tracers of very cold dust. Figure 7, composed of the $3.6 \mu \mathrm{m}$ IRAC (blue), $24 \mu \mathrm{m}$ MIPS (green), and $250 \mu \mathrm{m}$ SPIRE (red) images of the central $12^{\prime} \times 12^{\prime}$ area of L1340 C, reveals a complex network of filamentary dust formations.

The Akari Wide-L band image, centered on $140 \mu \mathrm{m}$, is displayed in Figure 8, with the contours of the visual extinction (Paper III) overplotted. The figure indicates that both the 
Table 5

Spitzer Sources of Class 0/I SED: Associated Objects

\begin{tabular}{|c|c|c|c|c|c|c|c|}
\hline SSTSL2 & SDSS DR9 J & 2MASS & AllWISE J & IRAS & Akari FIS & JCMTSF J & Other \\
\hline $022756.91+730354.4$ & $\ldots$ & $02275695+7303542$ & $022756.94+730354.6$ & $\ldots$ & $0227565+730402$ & $022756.6+730404$ & $\cdots$ \\
\hline $022800.65+730415.2$ & $022800.91+730415.4 \mathrm{~g}$ & $02280074+7304154$ & $022800.60+730415.4$ & $\cdots$ & $\ldots$ & $\ldots$ & $\ldots$ \\
\hline $022808.60+725904.5$ & $\ldots$ & $\ldots$ & $022808.59+725904.0$ & $\ldots$ & $0228071+725858$ & $022808.4+725902$ & $\cdots$ \\
\hline $022818.51+723506.2$ & $\ldots$ & $02281842+7235061$ & $022818.46+723506.3$ & $\ldots$ & $\ldots$ & $\ldots$ & $\ldots$ \\
\hline $022820.81+723500.5$ & $\ldots$ & $\ldots$ & $022820.76+723500.6$ & $\ldots$ & $0228201+723504$ & $\ldots$ & \\
\hline $022825.07+730945.6$ & $\ldots$ & $\ldots$ & $022825.06+730945.7$ & $\cdots$ & $\ldots$ & $\cdots$ & $\cdots$ \\
\hline $022842.57+723544.3$ & $022842.55+723544.5 \mathrm{~g}$ & $02284255+7235444$ & $022842.54+723544.5$ & $\ldots$ & $\ldots$ & $\ldots$ & $\ldots$ \\
\hline $022844.40+723533.5$ & $022844.40+723532.9 \mathrm{~g}$ & $02284443+7235332$ & $022844.39+723533.6$ & $\cdots$ & $\cdots$ & $\cdots$ & $\cdots$ \\
\hline $022844.71+730308.5$ & $\ldots$ & $\ldots$ & $022844.66+730308.6$ & $\ldots$ & $\ldots$ & $\ldots$ & $\ldots$ \\
\hline $022849.44+723731.6$ & $\ldots$ & $\cdots$ & $022849.40+723732.5$ & $\ldots$ & $\cdots$ & $\cdots$ & $\cdots$ \\
\hline $022855.69+731333.1$ & $\ldots$ & $\ldots$ & $022855.89+731333.1$ & $02240+7259$ & $\ldots$ & $\ldots$ & $\ldots$ \\
\hline $022856.61+730903.2$ & $\ldots$ & $\ldots$ & $022856.62+730903.1$ & $\ldots$ & $\ldots$ & $\ldots$ & $\ldots$ \\
\hline $022906.09+730210.5$ & $\ldots$ & $\ldots$ & $\ldots$ & $\ldots$ & $\ldots$ & $\cdots$ & $\ldots$ \\
\hline $022914.62+730102.8$ & $\cdots$ & $\cdots$ & $022914.67+725405.5$ & $\cdots$ & $\cdots$ & $\cdots$ & $\cdots$ \\
\hline $022918.25+724754.0$ & $\cdots$ & $\ldots$ & $\ldots$ & $\ldots$ & $\ldots$ & $\ldots$ & $\cdots$ \\
\hline $022931.98+725912.4$ & $\ldots$ & $02293228+7259130$ & $022932.05+725913.0$ & $02248+7245$ & $0229320+725911$ & $022933.2+725914$ & \\
\hline $022932.31+725503.2$ & $022932.32+725503.3^{*}$ & $\ldots$ & $022932.30+725503.2$ & $\ldots$ & $\ldots$ & $\ldots$ & $\ldots$ \\
\hline $022943.01+724359.6$ & $022943.09+724359.7$ & $\ldots$ & $022943.58+724358.6$ & $02249+7230$ & $\ldots$ & $\ldots$ & $\mathrm{HH} 489 \mathrm{~S}$ \\
\hline $022943.64+724358.6$ & $022949.66+725325.8$ & $\ldots$ & $022943.58+724358.6$ & $02249+7230$ & $\ldots$ & $\ldots$ & HH 489S \\
\hline $022949.62+725326.1$ & $\ldots$ & $\ldots$ & $022949.65+725326.3$ & $\ldots$ & $\ldots$ & $\ldots$ & $\ldots$ \\
\hline $022955.10+730309.1$ & $\cdots$ & $02295507+7303094$ & $022955.11+730309.4$ & $\cdots$ & $\cdots$ & $\ldots$ & $\cdots$ \\
\hline $022956.90+730217.0$ & $\cdots$ & $\ldots$ & $022957.34+730211.1$ & $\ldots$ & $\ldots$ & $022956.9+730217$ & \\
\hline $023022.78+730459.0$ & $\ldots$ & $\ldots$ & $023022.79+730459.0$ & $\ldots$ & $\ldots$ & $022956.9+730217$ & \\
\hline $023030.42+725706.7$ & $\ldots$ & $\ldots$ & $023030.34+725707.0$ & $\ldots$ & $\ldots$ & $\ldots$ & $\cdots$ \\
\hline $023032.44+725918.0$ & $023032.47+725917.7$ & $02303247+7259177$ & $023032.46+725917.8$ & $02259+7246$ & $\ldots$ & $\ldots$ & RNO 8 \\
\hline $023035.51+730828.2$ & $\ldots$ & $\ldots$ & $023035.52+730828.5$ & $\ldots$ & $\ldots$ & $\ldots$ & $\ldots$ \\
\hline $023042.36+730305.1$ & $\ldots$ & $02304238+7303051$ & $023042.36+730305.2$ & $\ldots$ & $\ldots$ & $\ldots$ & $\ldots$ \\
\hline $023127.34+724012.9$ & $\ldots$ & $02312734+7240130$ & $023127.20+724015.5$ & $02267+7226$ & $0231270+724015$ & $\ldots$ & $\ldots$ \\
\hline $023134.23+725829.1$ & $\cdots$ & $\ldots$ & $023134.25+725828.8$ & $\ldots$ & $\ldots$ & $\cdots$ & $\cdots$ \\
\hline $023142.50+725740.4$ & $\cdots$ & $\ldots$ & $023142.44+725740.6$ & $\ldots$ & $\ldots$ & $\cdots$ & $\cdots$ \\
\hline $023146.58+723729.4$ & $\ldots$ & $\ldots$ & $023146.53+723729.9$ & $\cdots$ & $\ldots$ & $\ldots$ & $\ldots$ \\
\hline $023203.42+724131.7$ & $\ldots$ & $\cdots$ & $023203.38+724131.7$ & $\cdots$ & $\ldots$ & $\ldots$ & $\ldots$ \\
\hline $023207.96+723759.3$ & $\cdots$ & $\cdots$ & $023207.88+723759.6$ & $\cdots$ & $\cdots$ & $\cdots$ & $\cdots$ \\
\hline $023225.98+724020.1$ & $\ldots$ & $\ldots$ & $023225.96+724020.3$ & $\cdots$ & $\cdots$ & $\cdots$ & $\cdots$ \\
\hline $023226.35+723919.4$ & $023226.57+723919.6 \mathrm{~g}$ & $02322653+7239198$ & $023226.37+723919.5$ & $\cdots$ & $\cdots$ & $\cdots$ & $\cdots$ \\
\hline $023227.64+723841.4$ & $\ldots$ & $\ldots$ & $023227.63+723841.4$ & $\cdots$ & $\cdots$ & $\cdots$ & $\cdots$ \\
\hline $023232.00+723827.5$ & $023231.70+723826.7$ & $02323198+7238280$ & $023231.92+723828.1$ & $\cdots$ & $\cdots$ & $\cdots$ & $\cdots$ \\
\hline $023237.90+723940.7$ & $\ldots$ & $\ldots$ & $023237.90+723940.7$ & $\cdots$ & $\ldots$ & $\ldots$ & $\ldots$ \\
\hline $023248.83+724635.4$ & $023248.85+724635.0 \mathrm{~g}$ & $02324885+7246369$ & $023248.77+724635.6$ & $\cdots$ & $\ldots$ & $\ldots$ & $\ldots$ \\
\hline $023256.14+724605.3$ & $\ldots$ & $02325605+7246055$ & $023256.14+724605.3$ & $\ldots$ & $0232567+724611$ & $\ldots$ & $\ldots$ \\
\hline $023302.41+724331.2$ & $\ldots$ & $02330247+7243315$ & $023302.41+724331.7$ & $02283+7230$ & $\ldots$ & $\ldots$ & [KOS94] HA11B \\
\hline $023330.92+724800.3$ & $\ldots$ & $\ldots$ & $023331.06+724800.7$ & $\ldots$ & $\ldots$ & $\cdots$ & $\ldots$ \\
\hline $023340.83+731950.8$ & $\ldots$ & $02334083+7319510$ & $023340.81+731950.8$ & $\cdots$ & $\ldots$ & $\ldots$ & $\ldots$ \\
\hline $023432.66+724057.2$ & $\ldots$ & $\ldots$ & $023432.75+724057.2$ & $\cdots$ & $\cdots$ & $\cdots$ & $\cdots$ \\
\hline $023532.06+724922.6$ & $\ldots$ & $\ldots$ & $023532.07+724922.8$ & $\cdots$ & $\ldots$ & $\ldots$ & $\cdots$ \\
\hline
\end{tabular}


Table 6

Spitzer Sources of Flat SED: Associated Objects

\begin{tabular}{|c|c|c|c|c|c|c|c|}
\hline SSTSL2 & SDSS DR9 J & 2MASS & AllWISE J & IRAS & Akari FIS & JCMTSF J & Other \\
\hline $022754.00+723535.5$ & $022753.97+723535.5^{*}$ & $02275399+7235354$ & $022753.96+723535.7$ & $\cdots$ & $\cdots$ & $\cdots$ & $\cdots$ \\
\hline $022759.92+723556.4$ & $022759.78+723555.6^{*}$ & $02275976+7235561$ & $022759.92+723556.4$ & $\ldots$ & $\ldots$ & $\ldots$ & HH $488 \mathrm{~S}$ \\
\hline $022811.32+723631.5$ & $022811.27+723631.6 \mathrm{~g}$ & $02281130+7236316$ & $022811.29+723631.6$ & $\cdots$ & $\cdots$ & $\ldots$ & $\ldots$ \\
\hline $022816.62+723732.6$ & $022816.63+723733.0^{*}$ & $02281661+7237328$ & $022816.62+723732.8$ & $02236+7224$ & $\cdots$ & $\ldots$ & [KOS94] HA 1, RNO 7-5 \\
\hline $022817.85+723800.9$ & $022817.85+723801.0^{*}$ & $02281782+7238009$ & $\ldots$ & $\ldots$ & $\cdots$ & $\cdots$ & [KOS94] HA 2, RNO 7-7 \\
\hline $022818.51+723734.6$ & $\ldots$ & $02281847+7237347$ & $\cdots$ & $\ldots$ & $\ldots$ & $\ldots$ & $\cdots$ \\
\hline $022838.02+723740.6$ & $022838.03+723740.8 \mathrm{~g}$ & $02283804+7237407$ & $022838.01+723740.8$ & $\cdots$ & $\cdots$ & $\ldots$ & $\cdots$ \\
\hline $022850.36+723851.2$ & $022850.35+723851.0^{*}$ & $02285031+7238506$ & $022850.29+723851.4$ & $\ldots$ & $\ldots$ & $\ldots$ & $\ldots$ \\
\hline $022851.83+723810.2$ & $022851.83+723810.1 \mathrm{~g}$ & $02285183+7238102$ & $022851.81+723810.2$ & $\ldots$ & $\ldots$ & $\ldots$ & $\ldots$ \\
\hline $022858.15+723801.4$ & $\ldots$ & $\ldots$ & $022858.06+723802.4$ & $\cdots$ & $\cdots$ & $\cdots$ & $\cdots$ \\
\hline $022907.88+724347.2$ & $\cdots$ & $02290783+7243475$ & $022907.89+724347.5$ & $\cdots$ & $\cdots$ & $\cdots$ & $\ldots$ \\
\hline $022917.57+723904.7$ & $\ldots$ & $02291777+7239045$ & $022917.49+723904.6$ & $\ldots$ & $\ldots$ & $\ldots$ & $\ldots$ \\
\hline $022919.60+730223.5$ & $022919.61+730223.6^{*}$ & $02291961+7302237$ & $022919.60+730223.7$ & F02246+7248 & $\ldots$ & $022921.2+730221$ & $\ldots$ \\
\hline $022920.70+730119.0$ & $022920.65+730119.6 \mathrm{~g}$ & $\ldots$ & $022920.66+730119.5$ & $\ldots$ & $\cdots$ & $\ldots$ & $\ldots$ \\
\hline $023020.61+730233.7$ & $023020.60+730233.8^{*}$ & $02302061+7302338$ & $023020.60+730233.9$ & $\ldots$ & $\ldots$ & $\ldots$ & $\ldots$ \\
\hline $023033.71+730125.1$ & $023033.68+730125.0^{*}$ & $\ldots$ & $023033.72+730125.1$ & $\cdots$ & $\cdots$ & $\ldots$ & $\cdots$ \\
\hline $023049.81+731049.2$ & $\ldots$ & $\ldots$ & $023049.72+731049.6$ & $\cdots$ & $\cdots$ & $\ldots$ & $\cdots$ \\
\hline $023053.25+730528.5$ & $\cdots$ & $\ldots$ & $023053.55+730528.1$ & $\ldots$ & $\ldots$ & $\ldots$ & $\ldots$ \\
\hline $023114.12+723933.3$ & $023114.07+723933.4^{*}$ & $\cdots$ & $023114.11+723933.3$ & $\cdots$ & $\ldots$ & $\cdots$ & $\cdots$ \\
\hline $023127.45+723912.8$ & $\ldots$ & $\cdots$ & $023127.40+723913.1$ & $\cdots$ & $\cdots$ & $\cdots$ & $\cdots$ \\
\hline $023127.52+725621.5$ & $\cdots$ & $\cdots$ & $\ldots$ & $\cdots$ & $\cdots$ & $\cdots$ & $\cdots$ \\
\hline $023134.62+725642.0$ & $023134.55+725640.8 \mathrm{~g}$ & $02313460+7256421$ & $023134.60+725642.2$ & $\cdots$ & $\cdots$ & $\cdots$ & $\cdots$ \\
\hline $023247.15+723858.8$ & $\ldots$ & $02324717+7238590$ & $023246.98+723859.0$ & $\cdots$ & $\cdots$ & $\cdots$ & $\cdots$ \\
\hline $023254.71+724257.9$ & $\cdots$ & $\ldots$ & & $\ldots$ & $\cdots$ & $\ldots$ & $\ldots$ \\
\hline $023301.52+724326.7$ & $023301.53+724326.8^{*}$ & $02330153+7243269$ & $023301.49+724327.0$ & $02283+7230$ & $\cdots$ & $\cdots$ & V1180 Cas \\
\hline
\end{tabular}




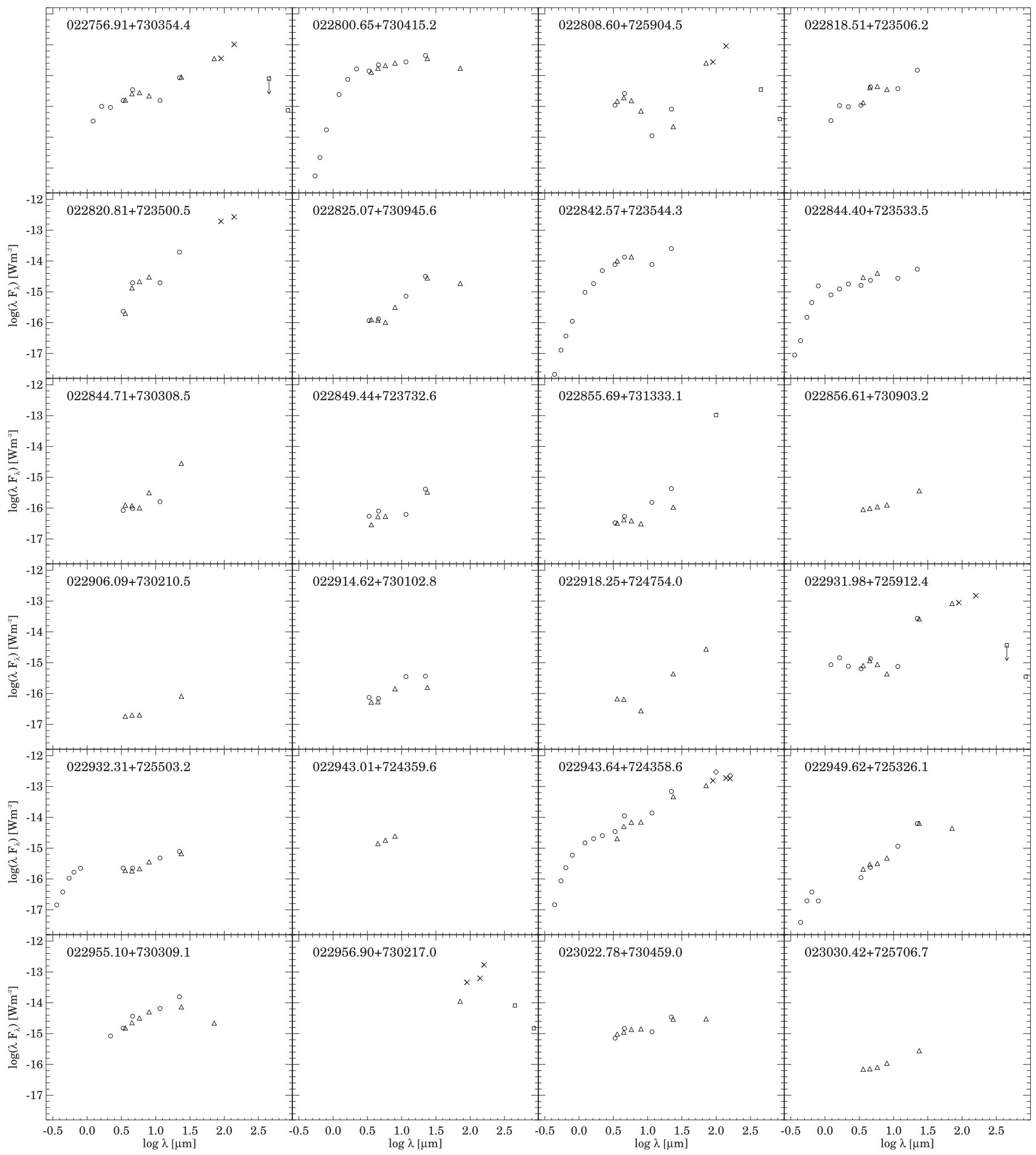

Figure 9. SEDs of Class I YSOs. Open circles show the SDSS, 2MASS, and WISE data, and triangles indicate the Spitzer data. Crosses are for Akari FIS data, diamonds show the Herschel data points, and squares indicate SCUBA submillimeter fluxes.

$140 \mu \mathrm{m}$ emission and the visual extinction trace the same component of the cloud. The lowest contour at $A_{\mathrm{V}}=1.0 \mathrm{mag}$ largely follows the $40-50 \mathrm{MJy} \mathrm{sr}^{-1}$ level of the far-infrared emission. At a few positions, heated by embedded YSOs, the strong $140 \mu \mathrm{m}$ emission is not associated with high extinction.

\section{YSOs IN L1340}

\subsection{Spitzer Sources}

\subsubsection{SED-based Classification}

We classified the candidate YSOs, selected by the color criteria described in Section 2.1, based on the slope of their 


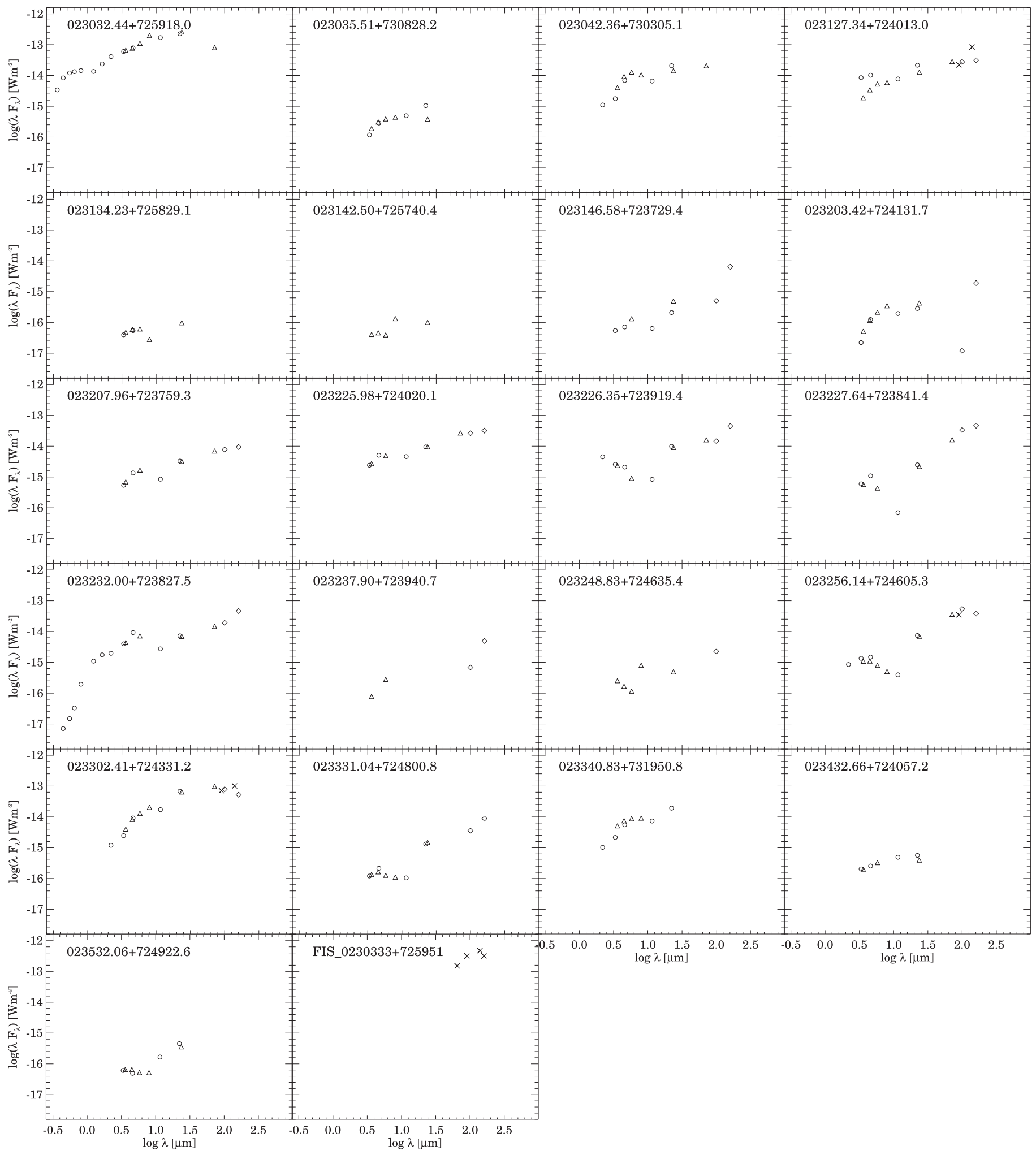

Figure 9. (Continued.)

SEDs, $\alpha=d \log (\lambda F(\lambda)) / d \log \lambda$. We derived $\alpha$ for both the $K_{\mathrm{s}}-24 \mu \mathrm{m}$ and the 3.6-8.0 $\mu \mathrm{m}$ intervals (for 3.6-5.8 $\mu \mathrm{m}$ when $8 \mu \mathrm{m}$ observations were missing). We used the WISE $22 \mu \mathrm{m}$ data when $24 \mu \mathrm{m}$ MIPS data were missing. According to the canonical classification scheme (Lada 1991; Greene et al.
1994), protostellar objects embedded in an envelope have $\alpha(2-24)>0.3$, whereas $\alpha(2-24)<-0.3$ for pre-mainsequence stars surrounded by accretion disks. Flat SED sources with $-0.3 \leqslant \alpha(2.0-24.0) \leqslant 0.3$ represent the transition between the protostellar and pre-main-sequence evolutionary 


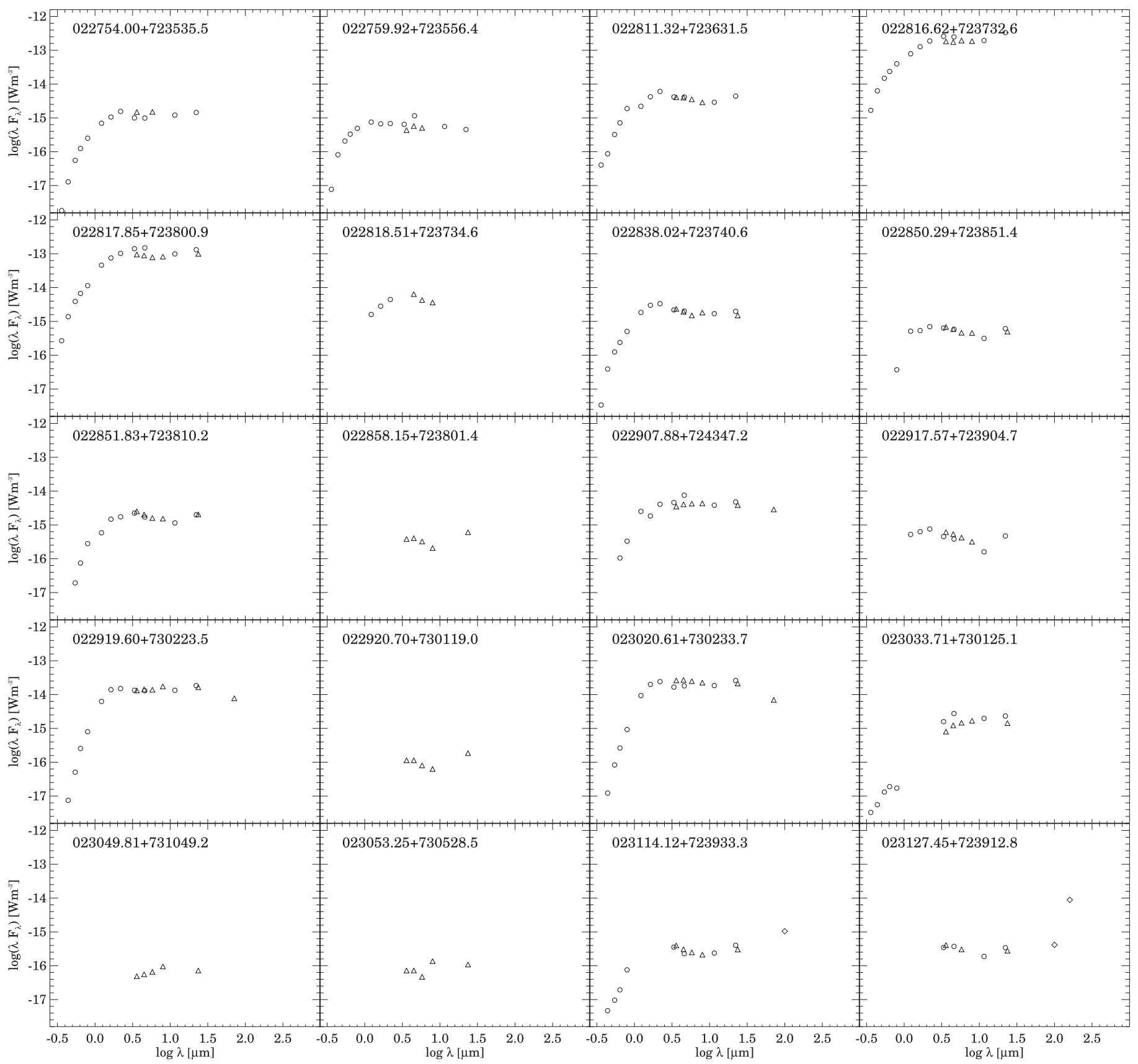

Figure 10. SEDs of the flat SED infrared sources. Symbols are the same as in Figure 9.

phases. We classified 155 Class II, 45 Class I, and 25 Flat SED sources. We detected a further Class I/Class 0 source in the $70 \mu \mathrm{m}$ MIPS image at $02^{\mathrm{h}} 29^{\mathrm{m}} 56^{\mathrm{s}} .90,+73^{\circ} 02^{\prime} 17 !^{\prime \prime} 0$. This source is undetectable at shorter wavelengths and coincides in position with an Akari FIS source and with a submillimeter source.

\subsubsection{Estimating Foreground Extinction}

Since the classification based on observed spectral slopes is biased by the extinction of the sources, we estimated the foreground extinctions of the candidate YSOs and then reclassified them according to the extinction-corrected SED slopes. Foreground extinctions of Class I and Flat SED sources were estimated using the extinction map, derived from SDSS star counts in Paper III. We adopted the pixel value of the extinction map at the position of the source as the foreground extinction of an embedded source. On the one hand, the extinction obtained in this manner is an upper limit, since the sources may be situated at any depth within the dusty medium. On the other hand, small-scale, high-extinction cores, missed by the extinction mapping, may be present around embedded sources. For the Class II sources we invoked SDSS and 2MASS counterparts. We compared the optical and nearinfrared side (from the $B$ to the $J$ band) of the SED with a grid of reddened photospheres, following the method described in Paper III, and thus estimated the spectral type and extinction of the central star. Based on the slopes of the extinction-corrected SEDs, two sources, classified originally as Class I, moved into the Flat class, and one Flat SED source moved into the Class II sample. Tables 1-3 list the SSTSL2 identifiers and 


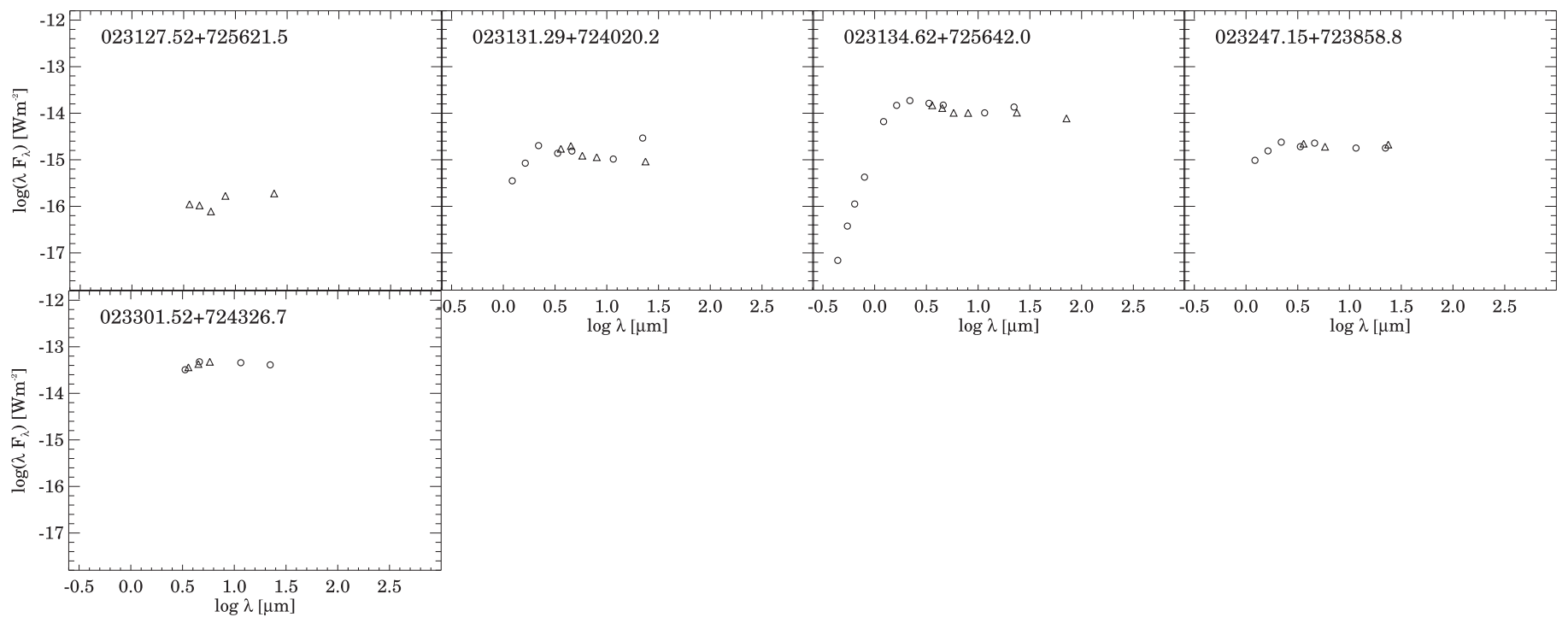

Figure 10. (Continued.)

Spitzer fluxes of the Class 0/I, Flat SED, and Class II sources of L1340, respectively.

The SEDs of Class II sources can be divided into further subclasses by comparing the dereddened SED slopes with the median band of the Taurus pre-main-sequence sample (D'Alessio et al. 1999; Furlan et al. 2006). The SED subclasses are indicative of the dust distribution in the circumstellar disks (Evans et al. 2009) of the classical T Tauri stars (CTTSs) and may shed light on the processes governing disk evolution. We classified the infrared excesses of our candidate pre-mainsequence stars into three groups: (1) the SED of primordial disks (II P subclass) does not drop below the Taurus median band; (2) the SED of the weak or anemic disks (II A subclass) is below the Taurus band over the whole observed wavelength region, and (3) pre-transitional and transitional disks (II T) have SEDs below the Taurus median band at intermediate wavelengths and start rising above $20 \mu \mathrm{m}$. For the latter group the spectral index $\alpha(8-24)>0$.

\subsubsection{Submillimeter, Far-infrared, and Optical Counterparts}

Six Spitzer sources are associated with submillimeter sources listed in the JCMT SCUBA Fundamental Catalog (Di Francesco et al. 2008). Far-infrared counterparts of 17 candidate Class 0/I and 3 Flat SED YSOs were identified in the Herschel PACS images. Table 4 lists the SSTSL2 associations and 100 and $160 \mu \mathrm{m}$ fluxes of these Herschel point sources.

Nine of the Spitzer-selected candidate YSOs coincide in position with far-infrared sources detected by the Akari/FIS instrument (Kawada et al. 2007). Four of them are included in the Akari/FIS YSO catalog (Tóth et al. 2014). A fifth catalog entry, Akari 0232291+723855, has an associated mid-infrared point source, AllWISE 023227.63+723841.4, within the halfmaximum radius of the PSF of the FIS (Arimatsu et al. 2014). Its fluxes, however, probably originate from more than one source. Similarly, the far-infrared fluxes of Akari FIS 0230333 +725951, a bright candidate YSO detected in each FIS band and associated with IRAS $02259+7246$, are composed of several sources. An extended emission can be seen around this position in the Spitzer $70 \mu \mathrm{m}$ image. We found SDSS counterparts of all but seven Class II infrared sources. A few Flat and Class I sources also have SDSS counterparts. Most of these counterparts are classified as galaxies. The nonstellar appearance, however, may indicate their scattered light origin.

SDSS, 2MASS, AllWISE, Akari, and other identifiers of Class I and flat sources are listed in Tables 5 and 6, respectively. For the Class II sample, excluding the 65 members common with the $\mathrm{H} \alpha$ emission stars studied in Paper III, we give the $U B V R_{\mathrm{C}} I_{\mathrm{C}} J H K_{\mathrm{s}}$ magnitudes in Table A1 of the Appendix.

The SEDs of the candidate YSOs, constructed from all available data, are displayed in Figures 9-11 for the Class I, Flat, and Class II sources, respectively. Since the SEDs of the $\mathrm{H} \alpha$ emission stars, together with those of the best-fitting photospheres, have been presented in Figure 9 of Paper III, Figure 11 presents the results for the Class II subsample not detected as $\mathrm{H} \alpha$ emission stars during our slitless spectroscopic $\mathrm{H} \alpha$ survey. The dereddened SEDs, as well as the best-fitting photosphere (Pecaut \& Mamajek 2013), are also plotted, and the derived spectral type and extinction are indicated in each plot.

\subsubsection{Bolometric Temperatures and Luminosities}

Bolometric temperatures and luminosities, as defined in Myers \& Ladd (1993), were derived from the dereddened SEDs for the Class I and Flat SED objects, detected at least in one band beyond $24 \mu \mathrm{m}$, and for the Class II sources, detected over the 0.36-24 $\mu \mathrm{m}$ region. Akari FIS, Herschel PACS, and JCMTSF submillimeter data were included in the integration when available. Contribution of the spectral regions beyond the longest wavelength was estimated using the method described by Chavarría-K (1981). The $L_{\mathrm{bol}}$ versus $T_{\mathrm{bol}}$ diagram of the candidate YSOs is plotted in Figure 12. The YSO classes, defined by the spectral slopes, correspond to the $T_{\text {bol }}$ intervals indicated in Figure 12 (Chen et al. 1995). It can be seen that both $\alpha(2-24)$ and $T_{\text {bol }}$ are consistent with the Class 0/I identification. Flat SED sources overlap in $T_{\text {bol }}$ with both Class I and Class II, whereas a significant part of the Class II sample has $T_{\text {bol }}$ above the theoretical boundary of 


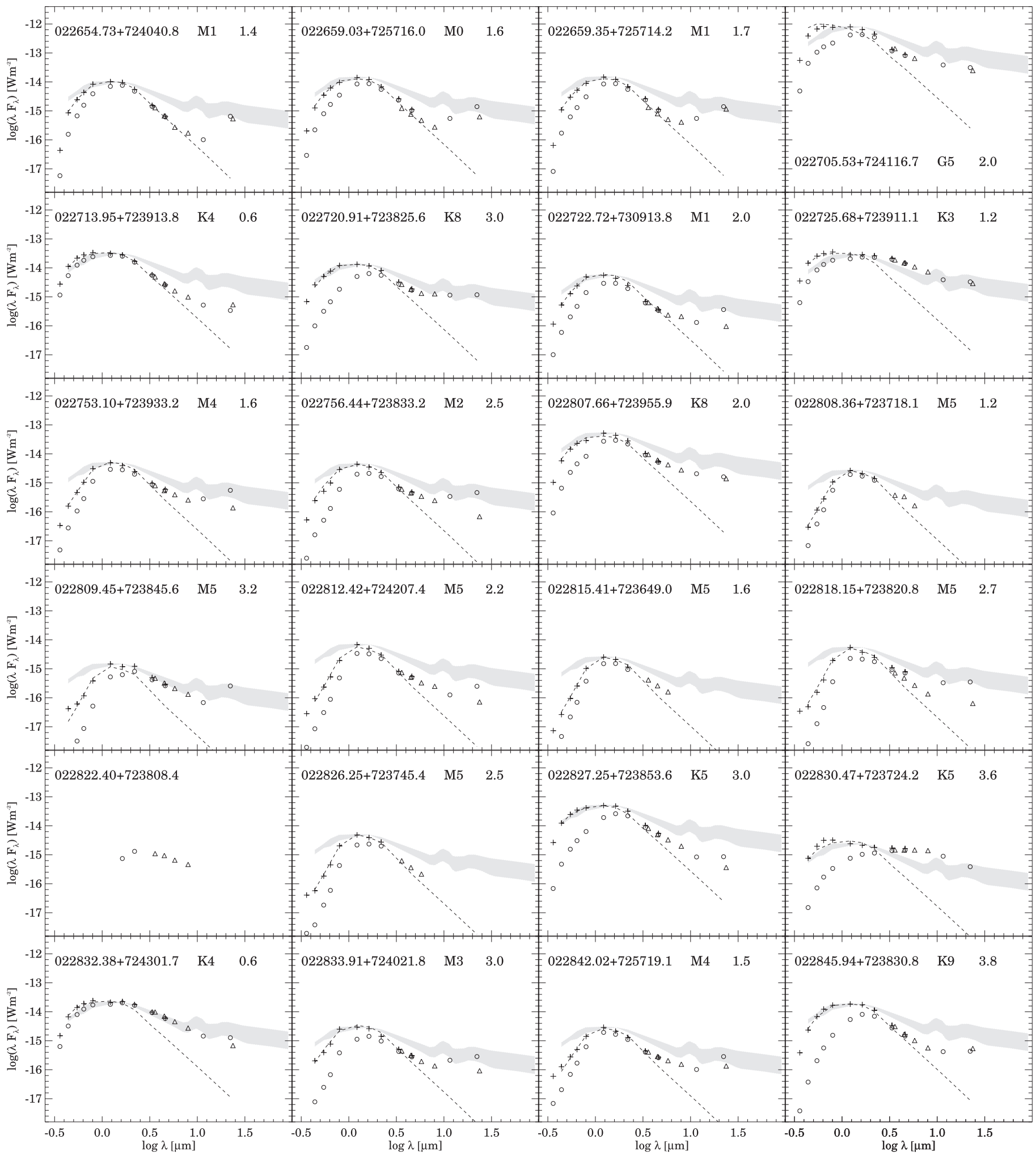

Figure 11. SEDs of the Class II candidate YSOs identified in the SEIP Source List data and not identified as H $\alpha$ emission stars. The SEDs of H $\alpha$ emission stars can be seen in Figure 9 of Paper III. Open circles show the SDSS, 2MASS, and WISE data, and triangles are for Spitzer data. Plus signs indicate the dereddened SED, and the dashed line shows the photospheric SED of the spectral type, obtained by fitting a model to the data. The gray shaded area indicates the median SED of the T Tauri stars of the Taurus SFR (D'Alessio et al. 1999). SSTSL2 identifiers, spectral types, and $A_{\mathrm{V}}$ extinctions, derived from photometric data, are indicated at the top of each panel.

$2800 \mathrm{~K}$. It is in accordance with the recent finding of Dunham et al. (2015) that the extinction-corrected $T_{\text {bol }}$ of a Class II source depends on the $T_{\text {eff }}$ of the central star, rather than on the disk properties. Figure 13 shows the histogram of bolometric luminosities of the candidate YSOs. The mean $L_{\text {bol }}$ of the 28 Class I sources, detected at $\lambda>24 \mu \mathrm{m}$, is $\left\langle L_{\text {bol,Classi }}\right\rangle=3.4 L_{\odot}$, and that for the Class II sample is $\left\langle L_{\text {bol,ClassII }}\right\rangle=1.2 L_{\odot}$. 


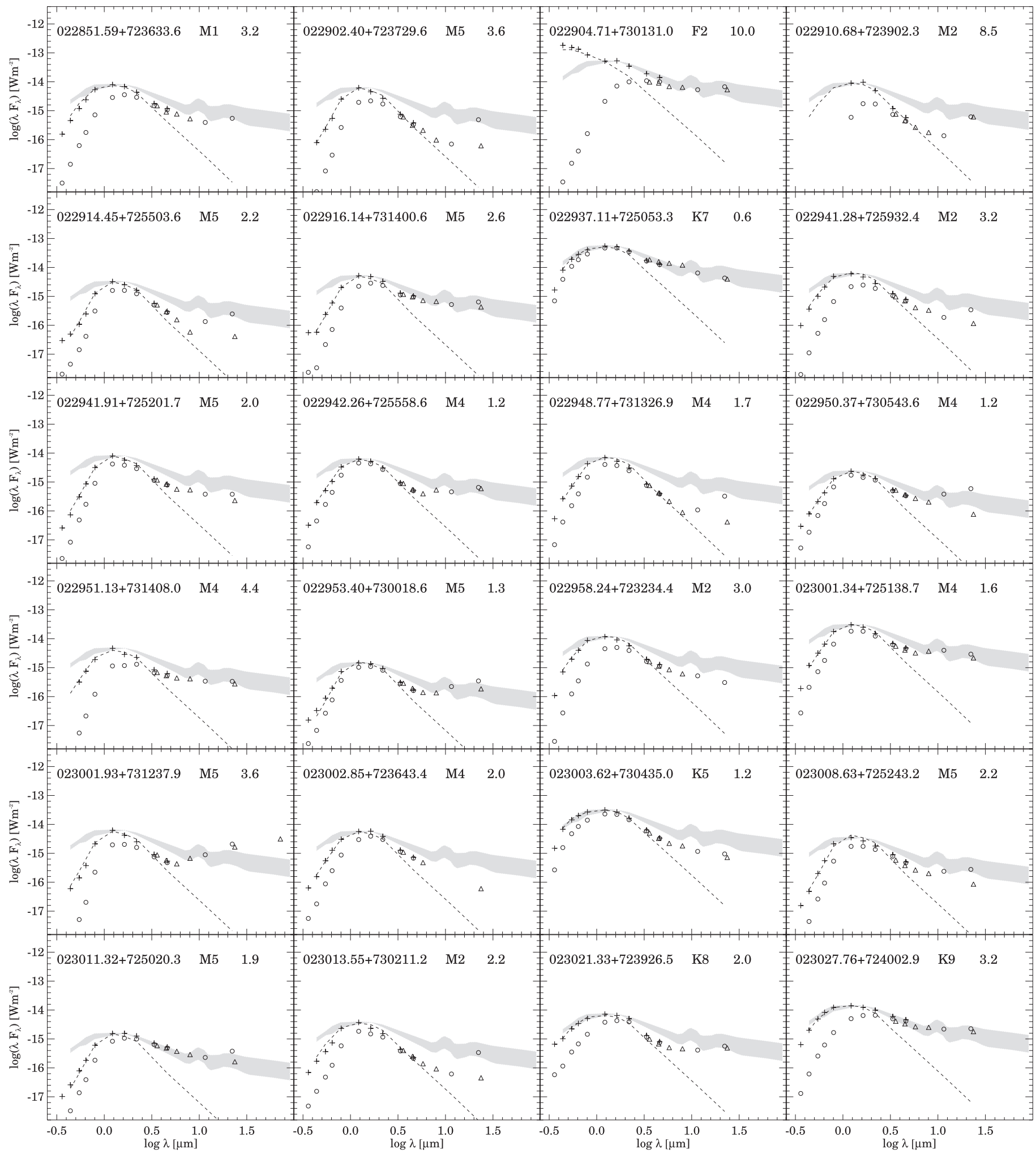

Figure 11. (Continued.)

Tables 7 and 8 present the derived extinctions, extinctioncorrected SED slopes, bolometric temperatures, and luminosities of Class 0/I and Flat SED sources, respectively. Table 9, in addition to the above quantities, lists the derived spectral types and luminosities for central stars of the Class II sources, as well as the SED subclasses.

\subsection{New Candidate Members of RNO 7 in the Omega-Cass Data}

The three-color composite of the $J$ (blue), $H$ (green), and $K$ (red) Omega-Cass images is shown in the second panel of Figure 14. For comparison, we show in the first panel an optical 


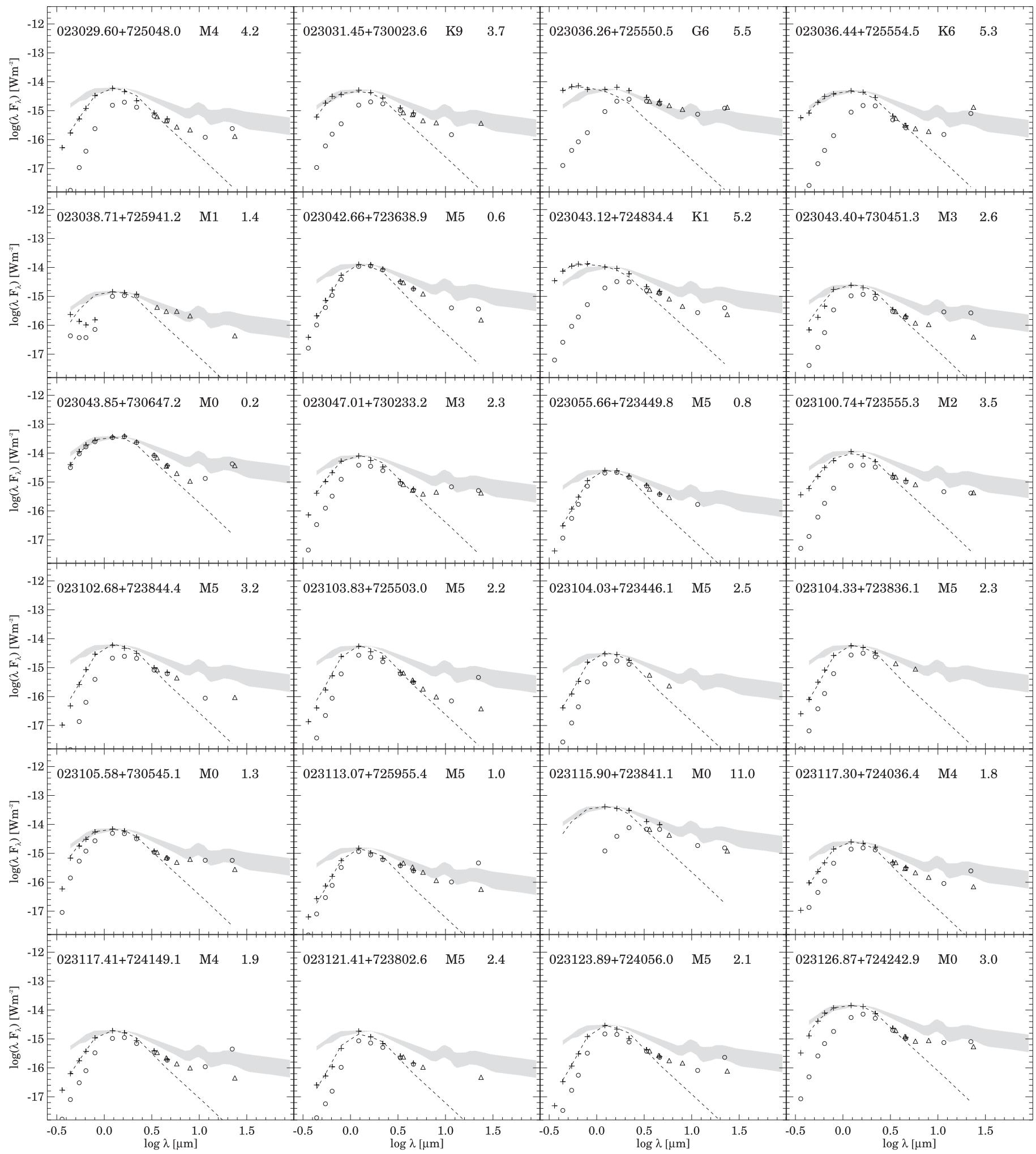

Figure 11. (Continued.)

three-color view of the same region, composed of the SDSS $g$ (blue), $r$ (green), and $i$ (red) images, whereas the third panel shows the Spitzer $3.6 \mu \mathrm{m}$ (blue), $4.5 \mu \mathrm{m}$ (green), and $8 \mu \mathrm{m}$ (red) composite image. The high angular resolution OmegaCass images reveal a few new objects, detectable neither in the optical nor in the IRAC images. Furthermore, they show that the brightest member of RNO 7, SSTSL2 J022816.62 +723732.6 , associated with IRAS $02236+7224$, has a faint companion at an angular distance of 1 "' 12 (Figure 14, fourth panel), corresponding to some 760 au at a distance of $825 \mathrm{pc}$.

The magnitudes measured in the Omega-Cass images and transformed into the 2MASS system are compared with the 


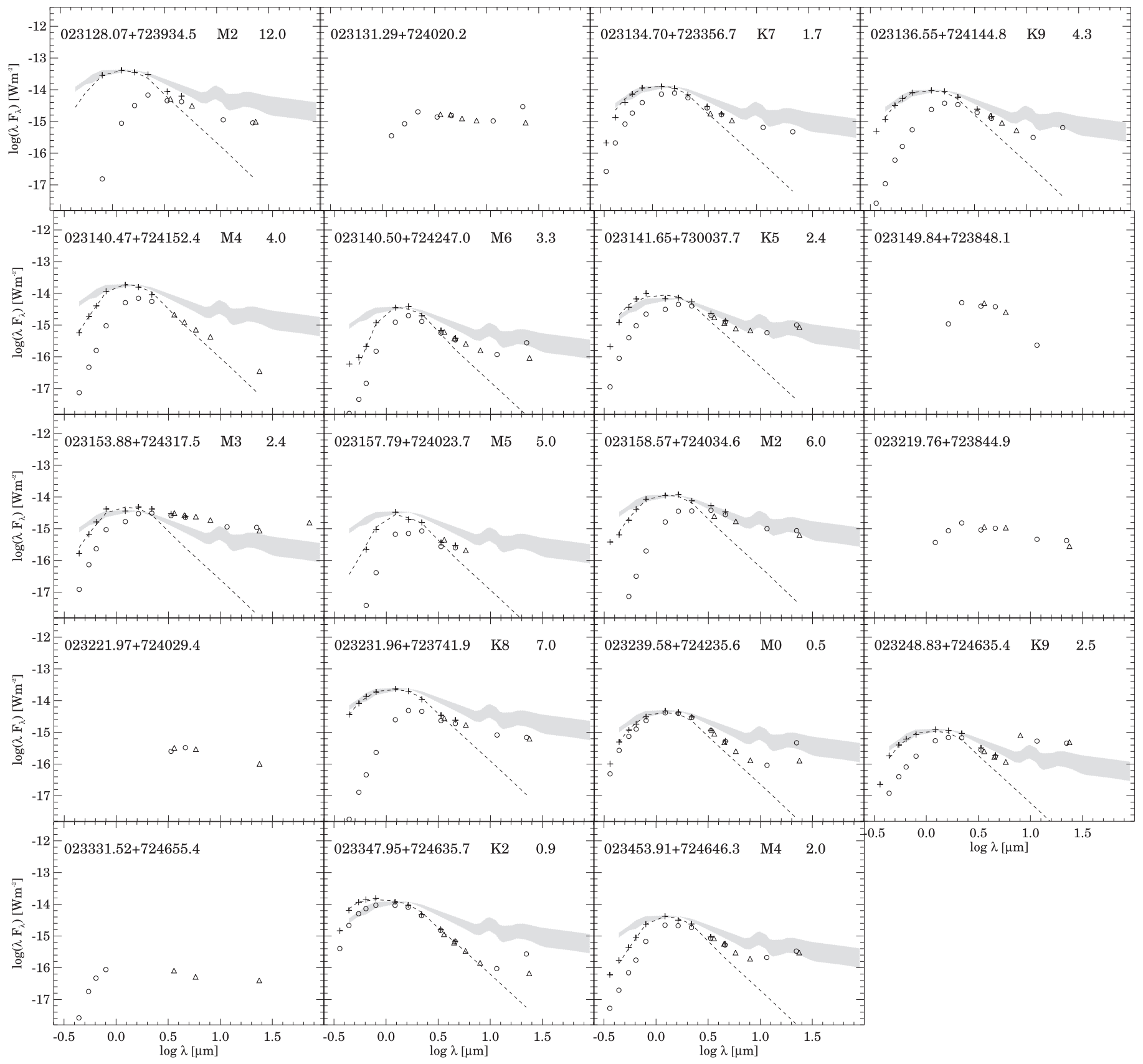

Figure 11. (Continued.)

2MASS magnitudes of the same stars in the left panel of Figure 15. The right panel of Figure 15 shows the $J-H$ versus $H-K_{\mathrm{s}}$ two-color diagram of the stars measured in each band. Twenty stars are located to the right of the band of the reddened normal main-sequence and giant stars, indicating $K_{\mathrm{s}}$-band excess. Table 10 lists the derived magnitudes of these stars. All but two of them have 2MASS counterparts, but none of them have good (A or B) photometric quality in each band. Six of the $14 \mathrm{H} \alpha$ emission stars, discovered by Magakian et al. (2003), and seven Spitzer-identified candidate YSOs are found in this sample. Eight stars, marked with asterisks in Table 10, are new candidate members of RNO 7. The SEDs of these eight stars, constructed from all available data, are presented in Figure 16.

\subsection{AllWISE Sources}

The 1 square degree area centered on R.A.(J2000) $=37^{\circ} .625$, decl. (J2000) $=+72.933$ contained 954 sources, having signalto-noise ratio greater than 5.0 in each band and not affected by the uppercase contamination flag. We identified seven new Class II source candidates in the WISE database outside the area covered by the Spitzer images, but within the lowest significant ${ }^{13} \mathrm{CO}$ contours of the molecular cloud. Each of them is located near the southern boundary of the cloud. Furthermore, two WISE sources without coinciding SSTSL2 entries, J022759.92 +723556.4 and J023227.63+723841.4, were found within the field of view of the Spitzer observations. We measured their fluxes in the available bands and added the sources to Tables 2 


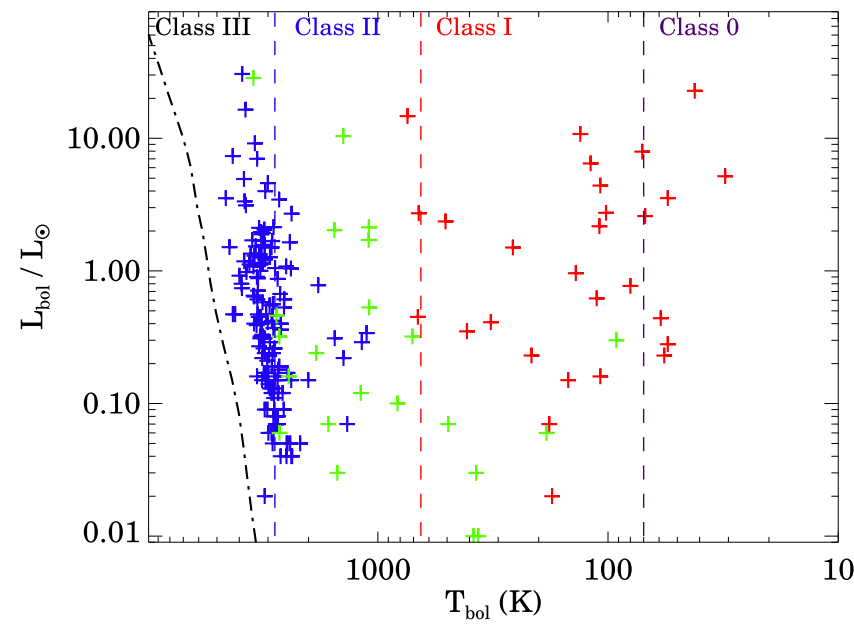

Figure 12. Extinction-corrected bolometric luminosities plotted agains extinction-corrected bolometric temperatures. Red plus signs indicate Class $0 /$ I sources, green plus signs are for Flat, and blue plus signs are for Class II SED slopes. Vertical dashed lines indicate the boundaries between the classes, and the dot-dashed line indicates the position of the zero-age main sequence (Siess et al. 2000).

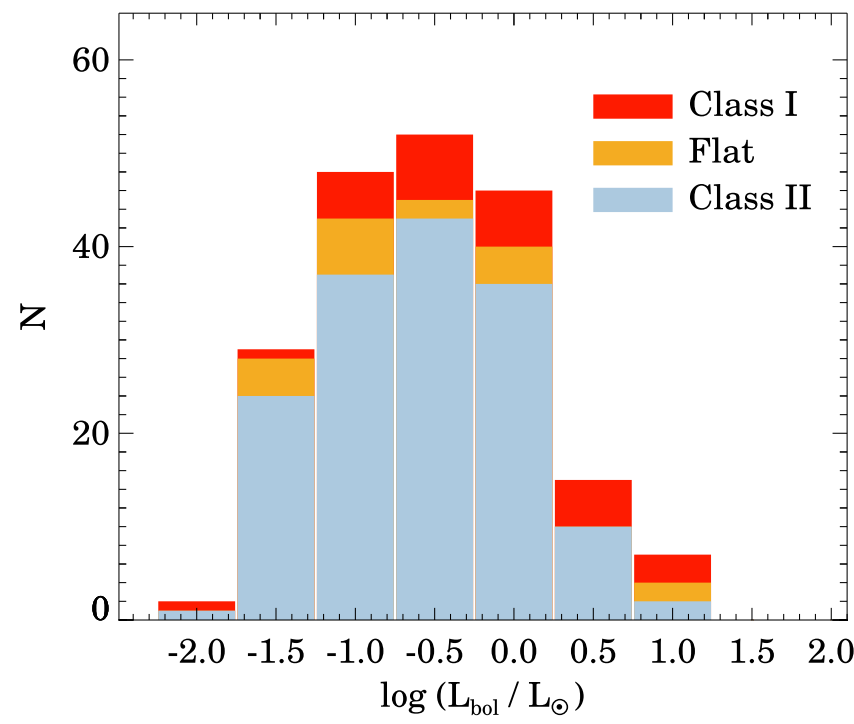

Figure 13. Histogram of bolometric luminosities of Class $0 / \mathrm{I} /$ Flat protostars and Class II pre-main-sequence stars.

and 1, respectively. The selected AllWISE sources are listed in Table 11. The SEDs of the seven WISE sources, identified as candidate YSOs outside the field of view of the Spitzer observations, are displayed in Figure 17. Each of them is a Class II source. Their $A_{\mathrm{V}}$ extinctions, spectral types, and luminosities derived from the photometric data are listed in Table 12.

\subsection{Embedded Protostars and Herbig-Haro Objects in L1340}

\subsubsection{Candidate Class 0 Sources}

The extinction-corrected SED slopes revealed the presence of 45 Class $0 / \mathrm{I}$ and 27 Flat SED candidate YSOs. Eight sources have $T_{\text {bol }} \lesssim 70 \mathrm{~K}$, suggesting the Class 0 evolutionary stage (Myers \& Ladd 1993). These are as follows.

1. SSTSL2 J022808.60+725904.5 coincides with an Akari FIS and a JCMTSF submillimeter source (see Table 5). Its SED, assembled from all available data (Figure 9), shows deep silicate absorption around $10 \mu \mathrm{m}$, suggesting a Class 0 protostar seen at high inclination (Enoch et al. 2009). This object is associated with a parsec-scale outflow identified in $\mathrm{H}_{2} 2.12 \mu \mathrm{m}$ observations (J. Walawender et al. 2016, in preparation). The three-color image of its environment, composed of IRAC $8 \mu \mathrm{m}$ (red), $4.5 \mu \mathrm{m}$ (green), and $3.6 \mu \mathrm{m}$ (blue) images and displayed in Figure 18, shows $4.5 \mu \mathrm{m}$ emission, originating from shocked $\mathrm{H}_{2}$.

2. SSTSL2 J022820.81+723500.5 lies outside the MIPS $70 \mu \mathrm{m}$ image. Its steeply rising SED is revealed by the Akari FIS data. With $L_{\mathrm{bol}} \approx 23 L_{\odot}$, it is the most luminous protostar of L1340. This source, together with another nearby Class I source, $022818.51+723506.2$, is located along the chain of Herbig-Haro objects $\mathrm{HH} 488$, whose several knots were detected in optical $\mathrm{H} \alpha$ and $\mathrm{S}_{\text {II }}$ images by Kumar et al. (2003) and Magakian et al. (2003). Kumar et al. (2003) suggested that the driving source was the brighter component of a binary star located at $2^{\mathrm{h}} 28^{\mathrm{m}} 00^{\mathrm{s}}, 72^{\circ} 35^{\prime} 58^{\prime \prime}(\mathrm{HH} 488 \mathrm{~S}$, Source 2 in Table 2). The optical counterpart of $\mathrm{HH} 488 \mathrm{~S}$ is classified as a galaxy in the SDSS DR9 and as an HH object by Magakian et al. (2003). Our photometry suggests a Flat SED, although it results from the composite fluxes of the central objects. The positions of the two protostars with respect to the HH knots suggest that either of them is the probable driving source. The IRAC images reveal new knots of $\mathrm{HH} 488$. In the upper panel of Figure 19 we marked the known and new knots of $\mathrm{HH} 488$ and the candidate driving sources. The lower panel shows a three-color composite image of $\mathrm{HH} 488$, whose angular extension of 5!6 corresponds to a total length of some $1.3 \mathrm{pc}$ at a distance of $825 \mathrm{pc}$.

3. SSTSL J022931.98+725912.4 is associated with IRAS and Akari far-infrared and a JCMTSF submillimeter source (Table 7). It is associated with a small fan-shaped reflection nebulosity, bright at $3.6 \mu \mathrm{m}$ on the eastern side, and a jet-like feature, bright at $4.5 \mu \mathrm{m}$ on the western side (Figure 20).

4. The fourth candidate Class 0 protostar is the $70 \mu \mathrm{m}$ source No. 22 in Table 1. It is associated with the brightest submillimeter source of the region.

5. SSTSL 023256.14+724605.3 is an embedded eruptive young star in L1340 C, discussed in Kun et al. (2014). Its $T_{\text {bol }}$ and $L_{\text {bol }}$ were determined including the Herschel 100 and $160 \mu \mathrm{m}$ fluxes.

6. SSTSL $023146.58+723729.4, \quad 023237.90+723940.7$, and $023330.92+724800.3$ are low-luminosity sources, not detected in the $70 \mu \mathrm{m}$ MIPS image. Their low bolometric temperatures were revealed by including the Herschel 100 and $160 \mu \mathrm{m}$ data in the SEDs. Their nature is uncertain: they may be either very low luminosity protostars or faint distant galaxies.

\subsubsection{Class I Protostars Associated with IRAS Sources}

Six IRAS sources, listed in Table 6 of Paper I, are associated with Class I Spitzer sources (see Table 5). IRAS 02249+7230 
Table 7

Extinctions, Extinction-corrected Spectral Indices, Bolometric Temperatures, and Luminosities of Class 0/I Sources of L1340

\begin{tabular}{|c|c|c|c|c|c|c|c|}
\hline SSTSL2 & $\begin{array}{c}A_{\mathrm{V}} \\
(\mathrm{mag})\end{array}$ & $\alpha(K-24)$ & $\alpha(3.6-24)$ & $\alpha(3.6-8.0)$ & $\alpha(24-70)$ & $\begin{array}{l}T_{\text {bol }} \\
(\mathrm{K})\end{array}$ & $\begin{array}{l}L_{\text {bol }} \\
\left(L_{\odot}\right) \\
\end{array}$ \\
\hline $022756.91+730354.4$ & 4.6 & 0.79 & 0.81 & 1.16 & 1.20 & 108 & 4.40 \\
\hline $022800.65+730415.2$ & 4.7 & 0.16 & 0.44 & 0.17 & -0.71 & 664 & 2.72 \\
\hline $022808.60+725904.5$ & 2.2 & $\cdots$ & -1.06 & -1.14 & 4.28 & 55 & 3.54 \\
\hline $022818.51+723506.2$ & 3.6 & $1.18^{\mathrm{a}}$ & $1.38^{\mathrm{b}}$ & 1.52 & $\ldots$ & $\ldots$ & $\ldots$ \\
\hline $022820.81+723500.5$ & 3.6 & $\cdots$ & $2.34^{\mathrm{b}}$ & 2.98 & $\ldots$ & 42 & 22.81 \\
\hline $022825.07+730945.6$ & 2.8 & $\cdots$ & 1.59 & 1.93 & -0.41 & 149 & 0.15 \\
\hline $022842.57+723544.3$ & 1.7 & $0.71^{\mathrm{a}}$ & $0.63^{b}$ & 0.14 & $\cdots$ & $\cdots$ & $\cdots$ \\
\hline $022844.40+723533.5$ & 1.7 & $0.48^{\mathrm{a}}$ & $0.64^{\mathrm{b}}$ & $\cdots$ & $\cdots$ & $\cdots$ & \\
\hline $022844.71+730308.5$ & 1.8 & $\cdots$ & 1.66 & -0.54 & $\cdots$ & $\cdots$ & $\cdots$ \\
\hline $022849.44+723731.6$ & 3.6 & $\cdots$ & 1.21 & 0.77 & $\cdots$ & $\cdots$ & $\cdots$ \\
\hline $022855.69+731333.1$ & 1.3 & $\cdots$ & 0.60 & 1.10 & $\cdots$ & 340 & 0.01 \\
\hline $022856.61+730903.2$ & 1.4 & $\cdots$ & 0.75 & 0.25 & $\cdots$ & $\cdots$ & $\cdots$ \\
\hline $022906.09+730210.5$ & 2.7 & $\cdots$ & 0.74 & 0.12 & $\cdots$ & $\cdots$ & \\
\hline $022914.62+730102.8$ & 3.1 & $\cdots$ & 0.52 & $\cdots$ & $\cdots$ & $\cdots$ & $\cdots$ \\
\hline $022918.25+724754.0$ & 1.1 & $\cdots$ & 0.97 & 2.51 & 1.66 & 82 & 0.11 \\
\hline $022931.98+725912.4$ & 1.5 & 1.43 & 1.82 & 3.73 & 1.02 & 71 & 7.93 \\
\hline $022932.31+725503.2$ & 1.7 & $\ldots$ & 0.64 & 0.53 & $\cdots$ & $\cdots$ & $\cdots$ \\
\hline $022943.01+724359.6$ & 1.4 & $\cdots$ & $\cdots$ & $\cdots$ & $\cdots$ & $\cdots$ & $\cdots$ \\
\hline $022943.64+724358.6$ & 2.5 & 1.14 & 1.61 & 1.68 & 0.72 & 132 & 10.77 \\
\hline $022949.62+725326.1$ & 2.5 & $\cdots$ & 1.74 & 2.29 & -0.39 & 410 & 0.35 \\
\hline $022955.10+730309.1$ & 2.8 & 0.77 & 0.74 & 0.22 & -1.14 & 323 & 0.41 \\
\hline $022956.90+730217.0$ & 4.2 & $\cdots$ & $\cdots$ & $\cdots$ & $\cdots$ & 31 & 5.17 \\
\hline $023022.78+730459.0$ & 3.3 & $\cdots$ & 0.50 & 0.55 & $\cdots$ & 215 & 0.23 \\
\hline $023030.42+725706.7$ & 3.9 & 0.66 & 0.65 & 0.15 & $\cdots$ & $\cdots$ & $\cdots$ \\
\hline $023032.44+725918.0$ & 3.3 & 0.79 & 0.72 & 0.65 & -1.06 & 743 & 14.69 \\
\hline $023035.51+730828.2$ & 1.2 & $\cdots$ & 0.32 & 1.07 & $\cdots$ & $\cdots$ & $\cdots$ \\
\hline $023042.36+730305.1$ & 2.4 & 1.00 & 0.62 & 0.23 & 0.31 & 259 & 1.50 \\
\hline $023127.34+724012.9$ & 2.2 & $\ldots$ & 0.96 & 0.64 & 0.71 & 102 & 2.74 \\
\hline $023134.23+725829.1$ & 2.1 & $\cdots$ & 0.31 & -0.65 & $\cdots$ & $\cdots$ & \\
\hline $023142.50+725740.4$ & 3.3 & $\cdots$ & 0.42 & 1.48 & $\cdots$ & $\cdots$ & $\cdots$ \\
\hline $023146.58+723729.4$ & 2.5 & $\cdots$ & $0.71^{b}$ & $\cdots$ & $\cdots$ & 57 & 0.23 \\
\hline $023203.42+724131.7$ & 3.9 & $\cdots$ & 1.67 & 1.16 & $\cdots$ & 180 & 0.07 \\
\hline $023207.96+723759.3$ & 2.3 & $\cdots$ & 0.70 & 1.10 & 0.64 & 112 & 0.62 \\
\hline $023225.98+724020.1$ & 5.2 & $\cdots$ & 0.61 & 0.75 & 0.91 & 109 & 2.17 \\
\hline $023226.35+723919.4$ & 2.4 & 0.16 & 0.63 & 0.71 & 0.48 & 138 & 0.96 \\
\hline $023227.64+723841.4$ & 3.8 & $\cdots$ & 0.58 & $\cdots$ & 1.74 & 80 & 0.77 \\
\hline $023232.00+723827.5$ & 6.0 & 0.36 & 0.14 & 0.62 & 0.62 & 508 & 2.36 \\
\hline $023237.90+723940.7$ & 4.8 & $\cdots$ & $\cdots$ & 1.60 & $\cdots$ & 55 & 0.28 \\
\hline $023248.83+724635.4$ & 2.2 & $\cdots$ & 0.35 & 1.44 & $\cdots$ & 108 & 0.16 \\
\hline $023256.14+724605.3$ & 1.6 & 0.84 & 0.95 & -0.38 & 1.48 & 69 & 2.59 \\
\hline $023302.41+724331.2$ & 1.7 & 1.58 & 1.41 & 1.50 & 0.38 & 119 & 6.46 \\
\hline $023330.92+724800.3$ & 2.8 & $\cdots$ & 1.21 & $\cdots$ & $\cdots$ & 59 & 0.44 \\
\hline $023340.83+731950.8$ & 3.3 & $1.26^{\mathrm{a}}$ & $1.15^{\mathrm{b}}$ & 0.67 & $\cdots$ & $\cdots$ & $\cdots$ \\
\hline $023432.66+724057.2$ & 2.2 & $\cdots$ & 0.36 & $\cdots$ & $\cdots$ & $\cdots$ & $\cdots$ \\
\hline $023532.06+724922.6$ & 1.5 & $\cdots$ & 0.90 & 1.02 & 1.78 & $\cdots$ & $\cdots$ \\
\hline
\end{tabular}

Notes.

${ }^{a} \alpha(K-22)$, since this source is outside of the MIPS images.

${ }^{\mathrm{b}} \alpha(3.4-22)$, since this source is outside of the MIPS images.

in L1340 A is the driving source of HH 489 (Magakian et al. 2003). The Spitzer data show it to be a wide binary, consisting of two Class I sources, SSTSL2 J022943.01 +724359.6 and SSTSL2 J022943.64+724358.6, separated by 2". 8. Their SEDs are shown in Figure 9, and the environment is displayed in the three-color image in Figure 21. HH 489 A, identified in optical $\mathrm{H} \alpha$ and $\mathrm{S}_{\text {II }}$ images by Magakian et al. (2003), and a chain of faint HH knots to the south can clearly be seen. Their projected distribution suggests that both components of the binary and another nearby Class I source, SSTSL2 J022950.37+724441.4, may contribute to their excitation.
Figure 12 shows that $T_{\text {bol }}$ of the second-brightest Class I object of L1340 falls into the Class II regime near the Class I/ Class II boundary. This ambiguous classification belongs to SSTSL2 J023032.44+725918.0, associated with IRAS 02259 +7246 and RNO 8. A faint optical star is visible at its position. Our low-resolution spectrum (Paper III) reveals its late G spectral type with the Balmer lines in emission, and the optical color indices point to an unreddened star. The bolometric luminosity, determined from the $I_{\mathrm{C}}$ or $J$ magnitudes, places this star near the ZAMS. All these data suggest the high inclination of the disk of this star. The optical and infrared images confirm this statement. Three-color images, shown in Figure 22, reveal 
Table 8

Extinctions, Extinction-corrected Spectral Indices, Bolometric Temperatures, and Luminosities of Flat SED Sources

\begin{tabular}{|c|c|c|c|c|c|c|}
\hline SSTSL2 & $\begin{array}{c}A_{\mathrm{V}} \\
(\mathrm{mag})\end{array}$ & $\alpha(K-24)$ & $\alpha(3.6-24)$ & $\alpha(3.6-8.0)$ & $\begin{array}{l}T_{\text {bol }} \\
(\mathrm{K})\end{array}$ & $\begin{array}{l}L_{\text {bol }} \\
\left(L_{\odot}\right)\end{array}$ \\
\hline $022754.00+723535.5$ & 3.3 & $-0.03^{\mathrm{a}}$ & $0.19^{\mathrm{b}}$ & $\cdots$ & 2393 & 0.15 \\
\hline $022759.92+723556.4$ & 0.9 & $-0.17^{\mathrm{a}}$ & $-0.19^{b}$ & $\ldots$ & 2674 & 0.06 \\
\hline $022811.32+723631.5$ & 2.6 & $-0.14^{\mathrm{a}}$ & $0.03^{\mathrm{b}}$ & -0.43 & 2726 & 0.43 \\
\hline $022816.62+723732.6$ & 3.8 & $0.25^{\mathrm{a}}$ & $0.13^{\mathrm{b}}$ & 0.02 & 3472 & 28.48 \\
\hline $022817.85+723800.9$ & 2.0 & 0.00 & 0.02 & -0.18 & 1377 & 10.16 \\
\hline $022818.51+723734.6$ & 3.6 & $\ldots$ & $\ldots$ & -0.14 & $\ldots$ & $\ldots$ \\
\hline $022838.02+723740.6$ & 3.5 & -0.32 & -0.24 & -0.32 & 2706 & 0.30 \\
\hline $022850.36+723851.2$ & 3.0 & -0.13 & -0.17 & -0.51 & $\ldots$ & $\cdots$ \\
\hline $022851.83+723810.2$ & 4.0 & 0.08 & -0.11 & -0.62 & 1647 & 0.22 \\
\hline $022858.15+723801.4$ & 3.2 & $\ldots$ & 0.24 & -0.78 & $\ldots$ & $\ldots$ \\
\hline $022907.88+724347.2$ & 2.9 & -0.01 & 0.05 & 0.27 & 1006 & 0.51 \\
\hline $022917.57+723904.7$ & 1.5 & $-0.20^{\mathrm{a}}$ & $0.02^{\mathrm{b}}$ & -0.81 & 1499 & 0.03 \\
\hline $022919.60+730223.5$ & 4.7 & 0.06 & 0.11 & 0.34 & 1378 & 1.87 \\
\hline $022920.70+730119.0$ & 3.1 & $\ldots$ & 0.26 & -0.73 & $\ldots$ & $\cdots$ \\
\hline $022950.37+724441.4$ & 3.8 & $\ldots$ & 0.28 & -0.23 & $\ldots$ & $\ldots$ \\
\hline $023020.61+730233.7$ & 2.2 & -0.04 & -0.11 & -0.19 & 1049 & 2.13 \\
\hline $023033.71+730125.1$ & 2.1 & $\ldots$ & 0.31 & 0.93 & 512 & 0.07 \\
\hline $023049.81+731049.2$ & 4.2 & $\ldots$ & 0.21 & 0.83 & $\ldots$ & $\ldots$ \\
\hline $023053.25+730528.5$ & 2.9 & $\ldots$ & 0.22 & 0.80 & $\ldots$ & $\ldots$ \\
\hline $023114.12+723933.3$ & 2.2 & $\cdots$ & -0.15 & -0.79 & 821 & 0.10 \\
\hline $023127.45+723912.8$ & 2.9 & $\ldots$ & -0.20 & $\ldots$ & 92 & 0.30 \\
\hline $023127.52+725621.5$ & 3.2 & $\ldots$ & 0.28 & 0.52 & $\ldots$ & $\ldots$ \\
\hline $023134.62+725642.0$ & 3.6 & -0.24 & -0.19 & -0.48 & 1096 & 1.71 \\
\hline $023247.15+723858.8$ & 3.6 & -0.04 & -0.02 & $\ldots$ & 1104 & 0.20 \\
\hline $023254.71+724257.9$ & 1.8 & $\ldots$ & 0.38 & -0.05 & $\ldots$ & $\ldots$ \\
\hline $023301.52+724326.7$ & 2.7 & $\ldots$ & $0.13^{\mathrm{b}}$ & $\ldots$ & 572 & 2.54 \\
\hline
\end{tabular}

Notes.

a $\alpha(K-22)$, since this source is outside of the MIPS images.

${ }^{\mathrm{b}} \alpha(3.6-22)$, since this source is outside of the MIPS images.

Table 9

Properties of Class II Objects Derived from the SEDs

\begin{tabular}{|c|c|c|c|c|c|c|c|c|c|c|}
\hline SSTSL2 & $\mathrm{Sp}$ & $\begin{array}{c}A_{\mathrm{V}} \\
(\mathrm{mag})\end{array}$ & $\begin{array}{l}T_{\text {eff }} \\
(\mathrm{K})\end{array}$ & $\begin{array}{l}L_{\text {star }} \\
\left(L_{\odot}\right)\end{array}$ & $\begin{array}{l}T_{\mathrm{bol}} \\
(\mathrm{K})\end{array}$ & $\begin{array}{l}L_{\text {bol }} \\
\left(L_{\odot}\right)\end{array}$ & $\alpha(K-24)$ & $\alpha(3.6-8.0)$ & $\alpha(8-24)$ & SED Subtype \\
\hline $022638.02+730457.5^{\mathrm{a}}$ & K4 & 0.7 & 4330 & 0.33 & 2556 & 0.56 & -0.76 & -0.38 & -0.77 & II P \\
\hline $022654.73+724040.8$ & M1 & 1.4 & 3630 & 0.30 & 3184 & 0.34 & -0.96 & -2.58 & 1.04 & II T \\
\hline $022659.03+725716.0$ & M0 & 1.6 & 3770 & 0.34 & 3198 & 0.41 & -0.96 & -1.94 & 0.75 & II T \\
\hline $022659.08+724016.6^{\mathrm{a}}$ & M1 & 1.5 & 3630 & 0.14 & 3183 & 0.16 & -1.15 & -1.18 & -0.73 & II A \\
\hline $022659.35+725714.2$ & M1 & 1.7 & 3630 & 0.33 & 3008 & 0.44 & -0.71 & -1.53 & 0.95 & II T \\
\hline $022700.34+724743.8^{\mathrm{a}}$ & K3 & 1.3 & 4550 & 0.79 & 3916 & 0.83 & -0.87 & -1.27 & 0.30 & II T \\
\hline $022702.11+724329.0^{\mathrm{a}}$ & K9 & 2.2 & 3770 & 0.41 & 3447 & 0.40 & -1.20 & -2.06 & -0.17 & II T \\
\hline $022703.17+723952.9^{\mathrm{a}}$ & M0 & 1.5 & 3770 & 0.13 & 3052 & 0.15 & -0.82 & -1.05 & -0.45 & II P \\
\hline $022705.53+724116.7$ & G5 & 2.0 & 5500 & 31.98 & 3882 & 31.14 & -1.18 & $\cdots$ & $\cdots$ & II A \\
\hline $022706.29+724011.1^{\mathrm{a}}$ & M0 & 1.8 & 3770 & 0.26 & 3232 & 0.32 & -1.15 & $\cdots$ & -0.10 & II A \\
\hline
\end{tabular}

Notes.

${ }^{\mathrm{a}} \mathrm{H} \alpha$ emission star.

b $\alpha(K-22)$, since this source is outside of the MIPS images.

c $\alpha(3.6-5.8)$.

d $\alpha(3.6-24)$.

(This table is available in its entirety in machine-readable form.)

the connections between various components of the circumstellar environment of RNO 8. The gap between the star and the nebulosity in the optical three-color image (first panel of Figure 22) suggests a huge shadow of the circumstellar disk on the dusty envelope, stretching far beyond the disk. The image composed of the optical $g$ (blue), IRAC $3.6 \mu \mathrm{m}$ (green), and
IRAC $8 \mu \mathrm{m}$ (red) shown in the second panel of Figure 22 reveals streaks of $8 \mu \mathrm{m}$ emission overlapping with the reflected starlight. The image in the third panel is composed of the $4.5 \mu \mathrm{m}$ (blue), $8 \mu \mathrm{m}$ (green), and $24 \mu \mathrm{m}$ (red) images. The overplotted contours of the $70 \mu \mathrm{m}$ emission reveal a cloud core associated with RNO 8. 

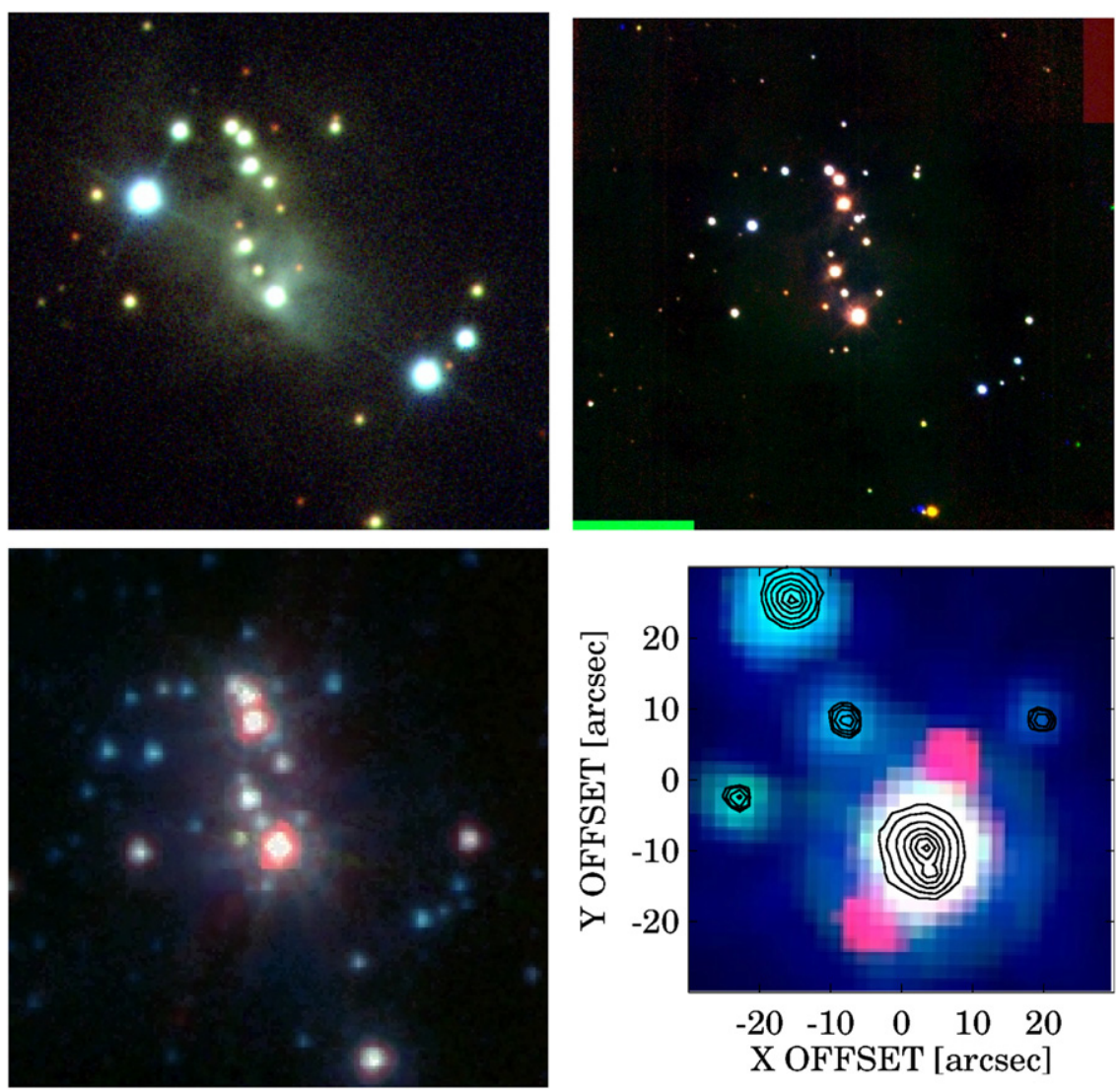

Figure 14. Optical, near-infrared, and mid-infrared three-color composite images of RNO 7. The upper left panel is composed of the SDSS $g$ (blue) $r$ (green), and $i$ (red) images; the upper right panel resulted from our high angular resolution $J$ (blue), $H$ (green), and $K$ (red) observations; and the lower left panel presents a composite of the Spitzer IRAC $3.6 \mu \mathrm{m}$ (blue), $4.5 \mu \mathrm{m}$ (green), and $8.0 \mu \mathrm{m}$ (red) images. The lower right panel magnifies a $30^{\prime \prime} \times 30^{\prime \prime}$ area of the previous image around the brightest member of the cluster. The IRAC composite image is scaled to show the two faint, $8 \mu \mathrm{m}$ companions, and Omega-Cass $K$-band contours, revealing a close near-infrared companion, are overplotted.
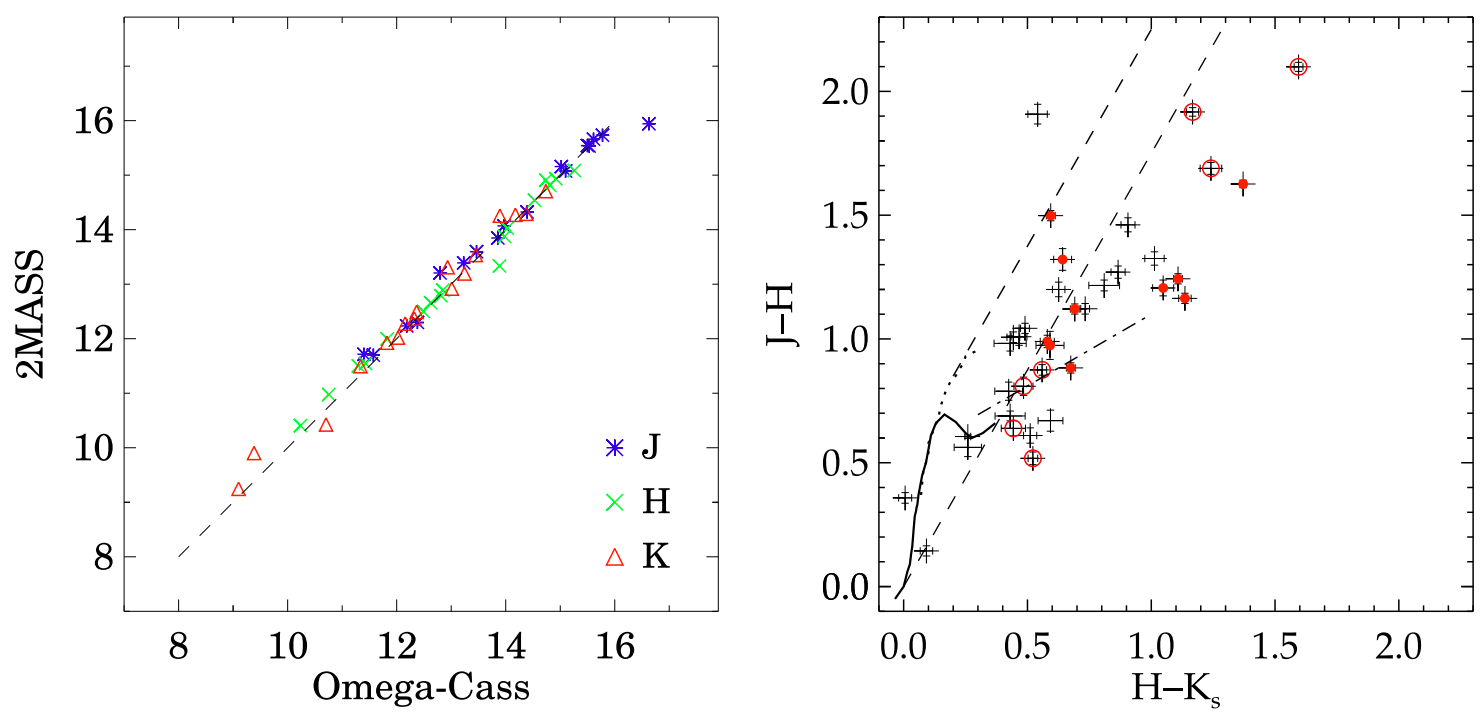

Figure 15. Left: 2MASS magnitudes of 17 stars within the field of view of Omega-Cass, plotted against those measured in the Omega-Cass images. Right: $J-H$ vs. $H-K_{\mathrm{S}}$ two-color diagram of the stars in the region of RNO 7, detected in each band in the Omega-Cass images, and listed in Table 10. The solid line indicates the colors of the main-sequence stars, and the dotted line shows those of the giants (Bessell \& Brett 1988). Dashed lines border the band of the reddened main-sequence and giant stars, and the dot-dashed line is the locus of T Tauri stars (Meyer et al. 1997). Red filled circles indicate the known H $\alpha$ emission stars, and open circles show the stars selected as Class II infrared sources in the Spitzer data. 
Table 10

NIR-excess Stars Identified in the Omega-Cass Images

\begin{tabular}{|c|c|c|c|c|c|}
\hline$N$ & $J \pm \Delta J$ & $H \pm \Delta H$ & $K \pm \Delta K$ & 2MASS & Other ID/Position \\
\hline 1 & $15.776 \pm 0.020$ & $15.258 \pm 0.016$ & $14.736 \pm 0.011$ & $02280836+7237183$ & SSTSL2 J022808.36+723718.1 \\
\hline 2 & $13.034 \pm 0.011$ & $11.828 \pm 0.030$ & $10.780 \pm 0.030$ & $02281182+7236447$ & RNO 7-3 \\
\hline $3^{\mathrm{a}}$ & $15.625 \pm 0.017$ & $15.192 \pm 0.024$ & $14.562 \pm 0.030$ & $\ldots$ & $022812.50+723644.0$ \\
\hline $4^{\mathrm{a}}$ & $14.631 \pm 0.020$ & $13.170 \pm 0.020$ & $12.264 \pm 0.020$ & $02281643+7237517$ & $\ldots$ \\
\hline $5^{\mathrm{a}}$ & $15.531 \pm 0.027$ & $14.921 \pm 0.015$ & $13.893 \pm 0.022$ & $02281673+7238090$ & $\cdots$ \\
\hline 6 & $11.400 \pm 0.025$ & $10.236 \pm 0.014$ & $9.100 \pm 0.011$ & $02281661+7237328$ & RNO 7-5 \\
\hline $7^{\mathrm{a}}$ & $17.097 \pm 0.018$ & $15.827 \pm 0.017$ & $14.961 \pm 0.023$ & $\ldots$ & $0228 \quad 17.35+723754.5$ \\
\hline $8^{\mathrm{a}}$ & $16.375 \pm 0.010$ & $15.050 \pm 0.015$ & $14.037 \pm 0.030$ & $02281720+7237243$ & $\cdots$ \\
\hline 9 & $13.969 \pm 0.025$ & $12.848 \pm 0.014$ & $12.157 \pm 0.011$ & $02281748+7237384$ & RNO 7-6 \\
\hline 10 & $12.381 \pm 0.025$ & $10.755 \pm 0.014$ & $9.384 \pm 0.011$ & $02281782+7238009$ & RNO 7-7 \\
\hline 11 & $12.497 \pm 0.025$ & $11.254 \pm 0.014$ & $10.146 \pm 0.011$ & $02281818+7238069$ & RNO 7-10 \\
\hline $12^{\mathrm{a}}$ & $17.012 \pm 0.015$ & $15.796 \pm 0.016$ & $14.986 \pm 0.060$ & $02281805+7237237$ & SSTSL2 $022818.13+723743.7$ \\
\hline $13^{\mathrm{a}}$ & $15.099 \pm 0.026$ & $13.977 \pm 0.014$ & $13.244 \pm 0.021$ & $02281840+7237479$ & $\cdots$ \\
\hline 14 & $12.737 \pm 0.015$ & $11.854 \pm 0.014$ & $11.179 \pm 0.011$ & $02281877+7238091$ & RNO 7-11 \\
\hline 15 & $16.628 \pm 0.012$ & $14.529 \pm 0.014$ & $12.934 \pm 0.001$ & $02281847+7237347$ & SSTSL2 J022818.51+723734.6 \\
\hline 16 & $17.589 \pm 0.017$ & $15.900 \pm 0.017$ & $14.659 \pm 0.040$ & $02282239+7238085$ & SSTSL2 J022822.40+723808.4 \\
\hline $17^{\mathrm{a}}$ & $16.818 \pm 0.012$ & $16.179 \pm 0.028$ & $15.736 \pm 0.040$ & $\ldots$ & SSTSL2 J022822.92+723830.5 \\
\hline 18 & $17.384 \pm 0.014$ & $15.467 \pm 0.010$ & $14.300 \pm 0.012$ & $02282402+7238065$ & SSTSL2 $022824.04+723806.4$ \\
\hline 19 & $15.611 \pm 0.016$ & $14.736 \pm 0.004$ & $14.177 \pm 0.012$ & $02282623+7237454$ & SSTSL2 $022826.25+723745.4$ \\
\hline 20 & $17.192 \pm 0.014$ & $16.383 \pm 0.008$ & $15.899 \pm 0.016$ & $02283047+7237245$ & SSTSL2 $022830.47+723724.2$ \\
\hline
\end{tabular}

Note.

${ }^{\mathrm{a}}$ New candidate YSO.

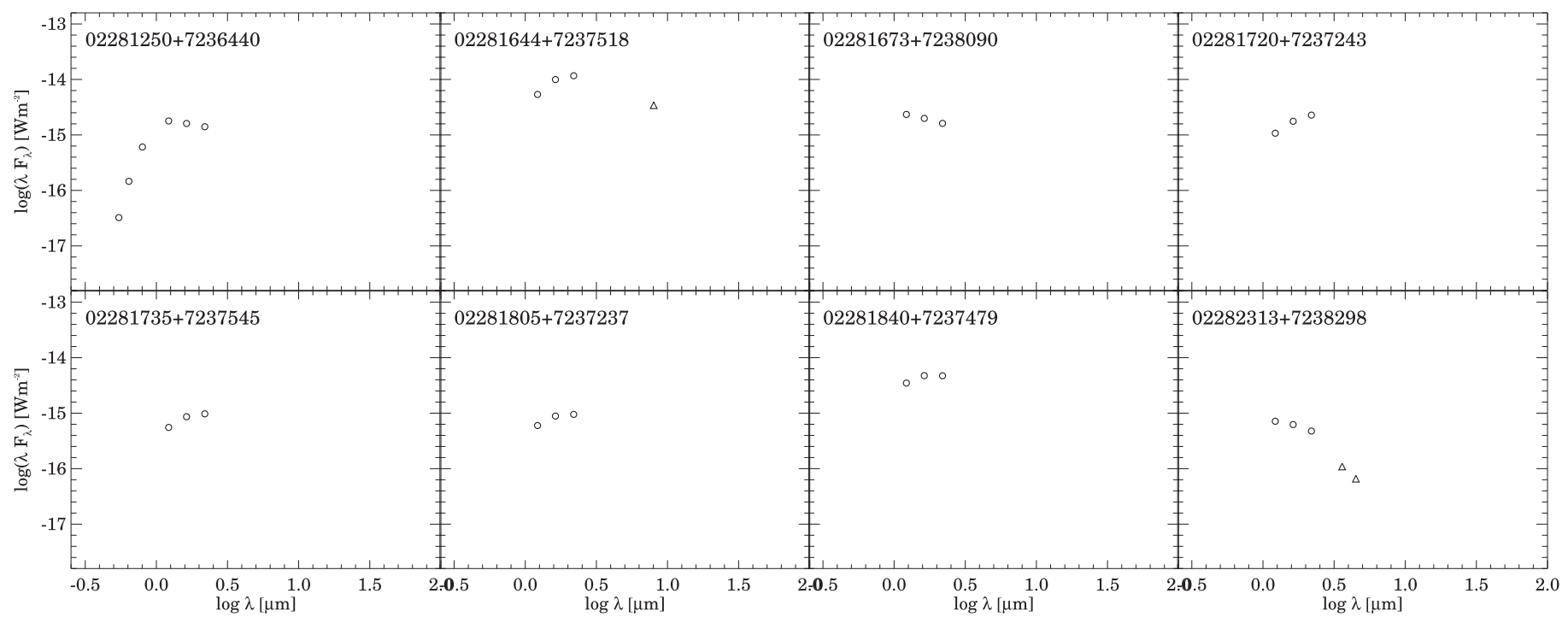

Figure 16. SEDs of the candidate young stars identified in the Omega-Cass $J H K$ data.

In L1340 C a J-shaped chain, consisting of five Class I and four Class II YSOs, can be seen close to the extinction peak (see Figure 29). IRAS $02276+7225$ and Akari FIS 0232291 +723855 are situated in the same area, but neither of them can be unambiguously associated with mid-infrared sources. Similarly, IRAS $02267+7226$ and Akari FIS 0231270 +724015 coincide with the Class I source SSTSL2 J023127.34+724012.9 within the position uncertainties, but other nearby sources may contribute to their cataloged fluxes.

SSTSL2 J023302.41+724331.2, coinciding with IRAS $02283+7230$, is the Class I companion of the eruptive star V1180 Cas. This protostar drives a jet, detected by Antoniucci et al. (2014) in [S II] and $\mathrm{H} \alpha$ narrowband images. The IRAC $4.5 \mu \mathrm{m}$ image also clearly shows the jet (Figure 23), as well as several faint $\mathrm{HH}$ objects. The $8 \mu \mathrm{m}$ image reveals a probable third component of the system, located at 4!"8 toward the north-northwest from V1180 Cas.

IRAS $02240+7259$, detected at $100 \mu \mathrm{m}$ only by IRAS and thus not listed in Paper I, coincides with a faint candidate protostar SSTSL2 J022855.69+731333.1, not detected at $70 \mu \mathrm{m}$. Taking into account the IRAS $100 \mu \mathrm{m}$ flux, the SED suggests a Class $0 / \mathrm{I}$ source with $T_{\mathrm{bol}} \approx 75 \mathrm{~K}$. The nature of this source, however, is uncertain: it may be a distant galaxy.

\subsection{Classical T Tauri Stars}

The Spitzer, WISE, and Omega-Cass data resulted in 170 Class II young stars in the region of L1340. These stars represent the CTTS population of L1340. Sixty-five of the 77 
Table 11

AllWISE Point Sources Selected as Candidate YSOs

\begin{tabular}{|c|c|c|c|c|}
\hline AllWISE & $\begin{array}{c}{[3.4]} \\
\text { (mag) }\end{array}$ & $\begin{array}{c}{[4.6]} \\
(\mathrm{mag})\end{array}$ & $\begin{array}{l}{[12.0]} \\
\text { (mag) }\end{array}$ & $\begin{array}{l}{[22.0]} \\
(\mathrm{mag})\end{array}$ \\
\hline $022759.92+723556.4^{\mathrm{a}, \mathrm{b}}$ & $14.060 \pm 0.026$ & $12.460 \pm 0.023$ & $10.328 \pm 0.076$ & $8.475 \pm 0.322$ \\
\hline $023037.18+723037.0^{\mathrm{a}}$ & $11.198 \pm 0.023$ & $10.652 \pm 0.021)$ & $8.699 \pm 0.026)$ & $6.673 \pm 0.076)$ \\
\hline $023043.49+722812.2$ & $10.593 \pm 0.024$ & $10.179 \pm 0.020)$ & $9.014 \pm 0.029)$ & $6.280 \pm 0.062)$ \\
\hline $023044.85+722639.8$ & $11.975 \pm 0.024$ & $11.503 \pm 0.021)$ & $10.095 \pm 0.054)$ & $8.145 \pm 0.199)$ \\
\hline $023147.07+722722.0$ & $11.360 \pm 0.022$ & $10.944 \pm 0.021)$ & $8.830 \pm 0.028)$ & $6.953 \pm 0.079)$ \\
\hline $023202.38+722820.3$ & $12.646 \pm 0.024$ & $12.103 \pm 0.023)$ & $10.272 \pm 0.075)$ & $7.656 \pm 0.145)$ \\
\hline $023209.62+722858.8$ & $11.206 \pm 0.022$ & $10.896 \pm 0.020)$ & $9.179 \pm 0.033)$ & $6.312 \pm 0.060)$ \\
\hline $023212.59+723013.7^{\mathrm{a}}$ & $11.086 \pm 0.023$ & $10.352 \pm 0.021)$ & $8.275 \pm 0.022)$ & $6.286 \pm 0.050)$ \\
\hline $023227.63+723841.4^{\mathrm{b}}$ & $14.231 \pm 0.027$ & $12.630 \pm 0.023$ & $12.249 \pm 0.339$ & $6.632 \pm 0.064$ \\
\hline
\end{tabular}

Notes.

${ }^{\mathrm{a}} \mathrm{H} \alpha$ emission star (Paper III).

${ }^{\mathrm{b}}$ This source is located within the field of view of the Spitzer observations, but missing from the SEIP Source List.

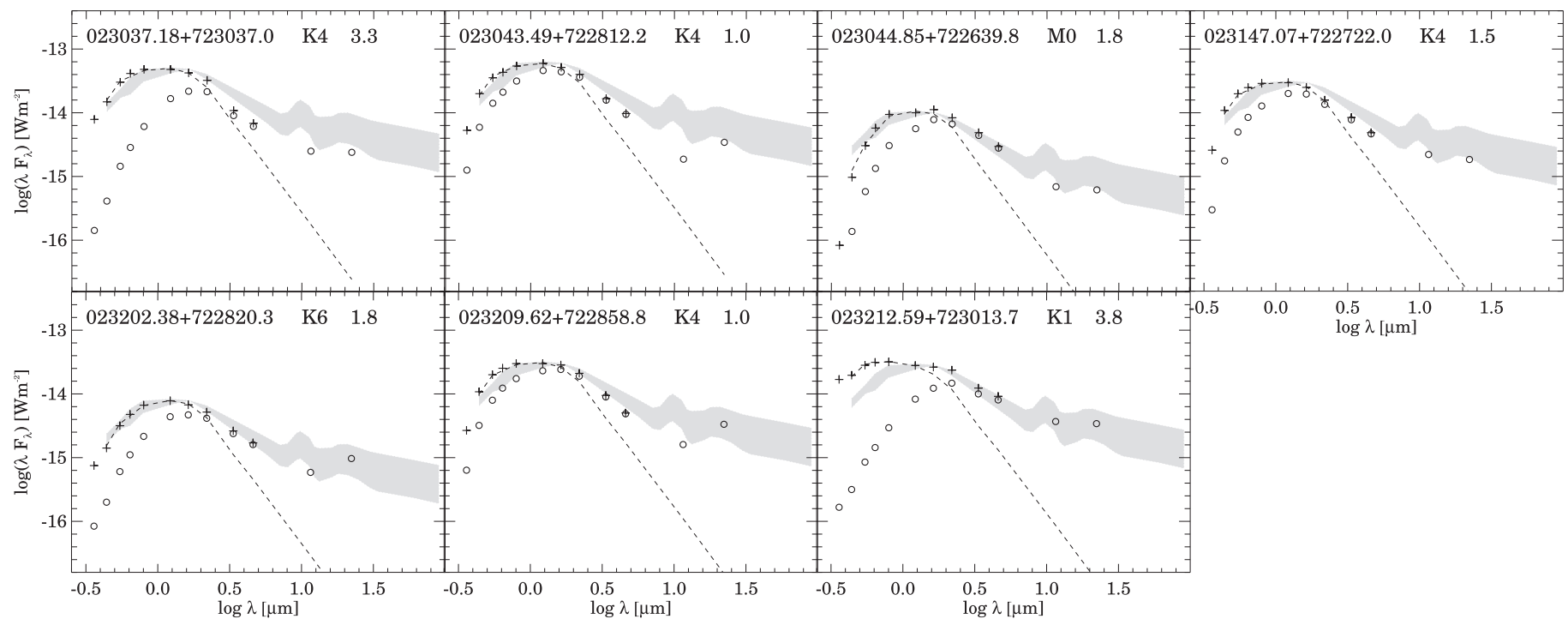

Figure 17. SEDs of the candidate young stars identified in the AllWISE data, located outside of the field of view of the Spitzer observations.

Table 12

Properties of Class II AllWISE Sources, Derived from the SEDs

\begin{tabular}{|c|c|c|c|c|c|c|}
\hline AllWISE & Sp. & $A_{\mathrm{V}}$ & $T_{\text {eff }}$ & $\left(L_{\text {star }} / L_{\odot}\right)$ & $\alpha(K-22)$ & SED Subtype \\
\hline $023037.18+723037.0$ & K4 & 3.3 & 4330 & 1.377 & -1.101 & II A \\
\hline $023043.49+722812.2$ & K4 & 1.0 & 4330 & 2.070 & -1.063 & II A \\
\hline $023044.85+722639.8$ & M0 & 1.8 & 3770 & 0.278 & -1.112 & II A \\
\hline $023147.07+722722.0$ & $\mathrm{~K} 4$ & 1.5 & 4330 & 1.064 & -0.930 & II A \\
\hline $023202.38+722820.3$ & K6 & 1.8 & 4020 & 0.222 & -0.713 & II T \\
\hline $023209.62+722858.8$ & K4 & 1.0 & 4330 & 1.089 & -0.797 & II T \\
\hline $023212.59+723013.7$ & $\mathrm{~K} 1$ & 3.8 & 4920 & 0.864 & -0.811 & II P \\
\hline
\end{tabular}

$\mathrm{H} \alpha$ emission stars, presented in Paper III, are members of this sample. These stars are marked with asterisks in Tables 3 and 9. Histograms of their $K_{\mathrm{s}}$ magnitudes and derived $A_{\mathrm{V}}$ and $T_{\text {eff }}$ values are shown in Figure 24, together with those of the $\mathrm{H} \alpha$ emission subset (Paper III). It can be seen that $\mathrm{H} \alpha$ emission was detected in brighter and hotter Class II stars. Only five of the Class II stars brighter than $K_{\mathrm{s}}=11.5$ were not detected during the $\mathrm{H} \alpha$ survey, and only one $\mathrm{H} \alpha$ emission star has 


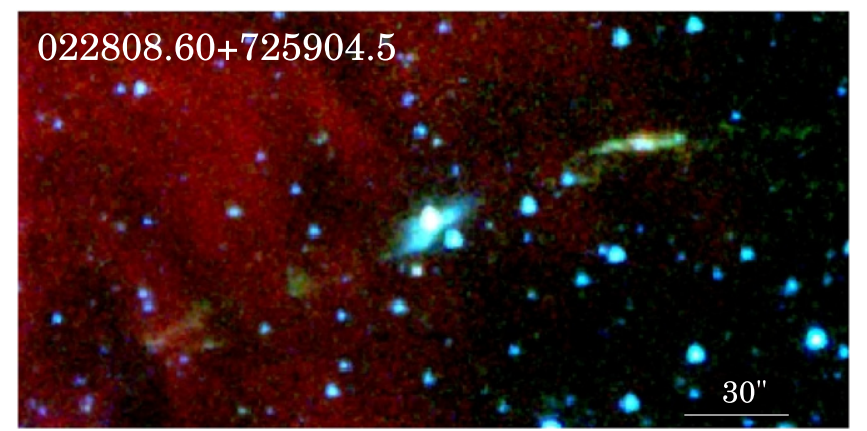

Figure 18. Three-color image composed from IRAC $8 \mu \mathrm{m}$ (red), $4.5 \mu \mathrm{m}$ (green), and $3.6 \mu \mathrm{m}$ (blue) images of the candidate Class 0 protostar SSTSL2 $\mathrm{J} 022808.60+725904.5$. The $4.5 \mu \mathrm{m}$ knots at both sides of the nebulous source indicate $\mathrm{HH}$ objects driven by the Class 0 protostar, and corresponding to the protostellar outflow reported in J. Walawender et al. (2016, in preparation).
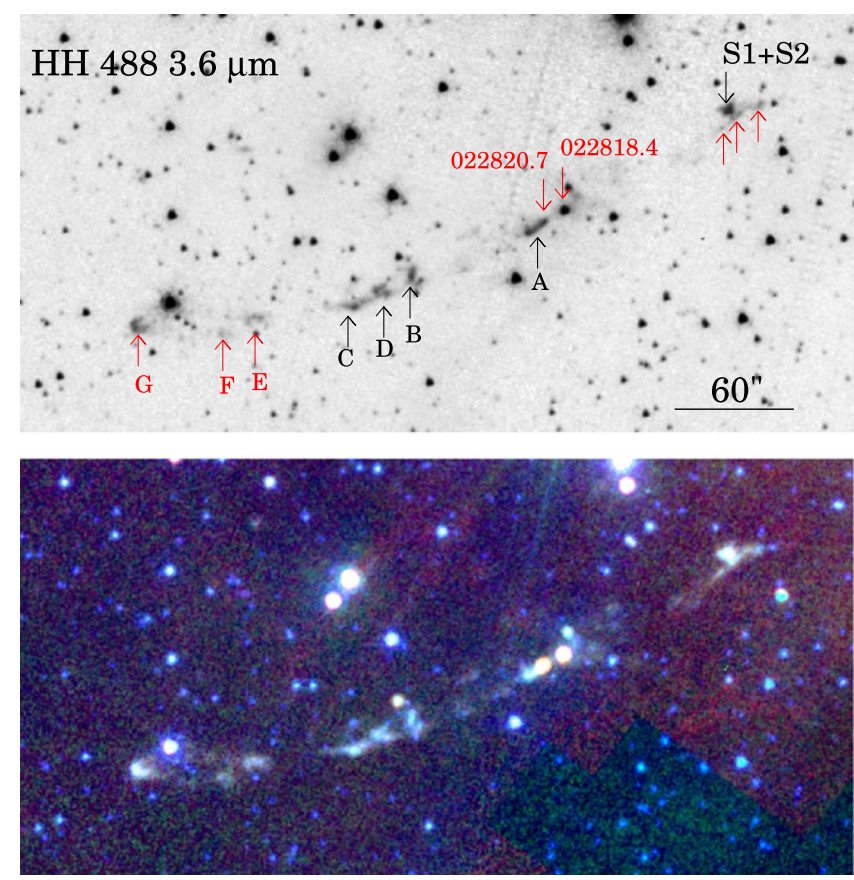

Figure 19. Top: HH 488 in the $3.6 \mu$ m IRAC band. The HH knots A, B, C, and $\mathrm{D}$ and the binary star $\mathrm{S} 1+\mathrm{S} 2$, the driving source candidate identified by Kumar et al. (2003), are indicated by the black characters, and red characters mark the new protostars SSTSL2 022818.51+723506.2 and SSTSL2 022820.81 +723500.5 and $\mathrm{HH}$ knots E, F, and G, revealed by the Spitzer images. Bottom: three-color image of the same region, composed from IRAC $8 \mu \mathrm{m}$ (red), $5.8 \mu \mathrm{m}$ (green), and $3.6 \mu \mathrm{m}$ (blue) images.

spectral type later than M2. The derived extinctions of the Class II sources peak between $2 \mathrm{mag}<A_{\mathrm{V}}<3 \mathrm{mag}$.

After estimating their spectral classes and extinctions, we plotted the positions of all candidate pre-main-sequence stars in the $\log T_{\text {eff }}-\log L$ plane. The intermediate-mass young main-sequence stars, identified in Paper III, are also plotted. Effective temperatures of the spectral types were adopted from Pecaut \& Mamajek (2013). Bolometric luminosities were derived from the extinction-corrected $I_{\mathrm{C}}$ and $J$ magnitudes, separately, using the bolometric corrections and color indices tabulated for pre-main-sequence stars by Pecaut \& Mamajek (2013), and adopting the distance of 825 pc.

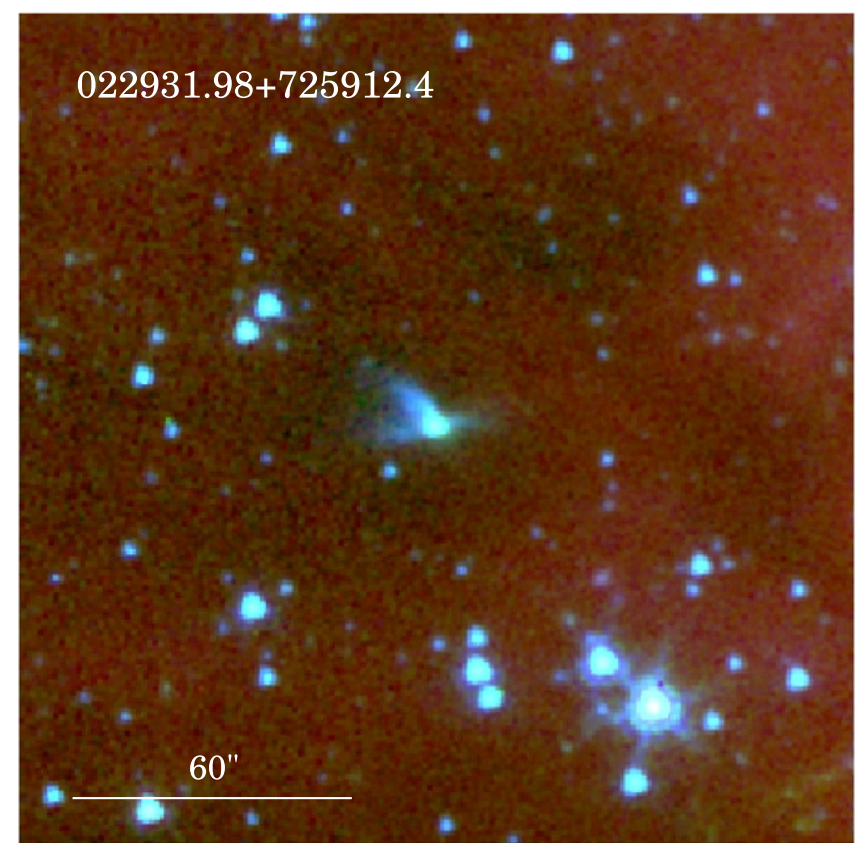

Figure 20. Three-color image, composed of the IRAC $3.6 \mu \mathrm{m}$ (blue), $4.5 \mu \mathrm{m}$ (green), and $8 \mu \mathrm{m}$ (red) images of the environment of the candidate Class 0 protostar SSTSL2 J022931.98+725912.4. Notice the color difference between the eastern and western nebulosities.

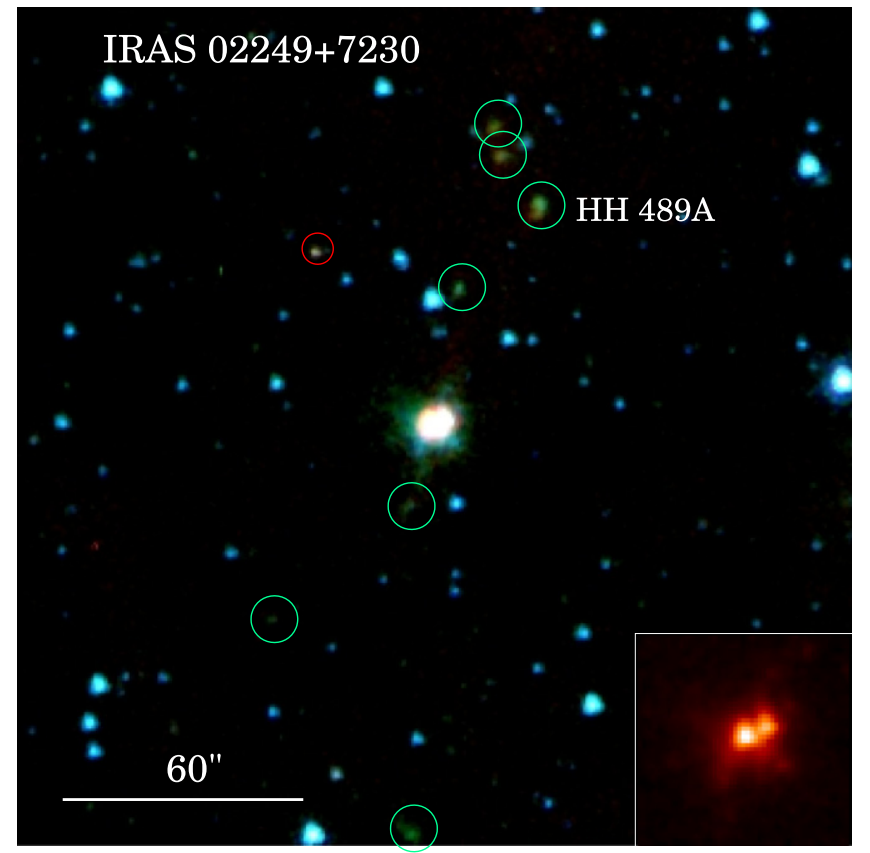

Figure 21. Three-color image, composed from the IRAC $3.6 \mu \mathrm{m}$ (blue), $4.5 \mu \mathrm{m}$ (green), and $8 \mu \mathrm{m}$ (red) images of the environment of the binary protostar IRAS $02249+7230$, the driving source of $\mathrm{HH} 489$. HH $489 \mathrm{~A}$ and further $\mathrm{HH}$ objects revealed by the $4.5 \mu \mathrm{m}$ image are marked by the green circles. The faint red object within the red circle is another Class I object, SSTSL2 J022950.37+724441.4. The inset in the lower right corner shows the 30 " environment of the central object, magnified and scaled for better visibility.

Finally, the results obtained from the $I_{\mathrm{C}}$ and $J$ magnitudes were averaged. Figure 25 shows the Hertzsprung-Russell diagram (HRD). Evolutionary tracks and isochrones for the 

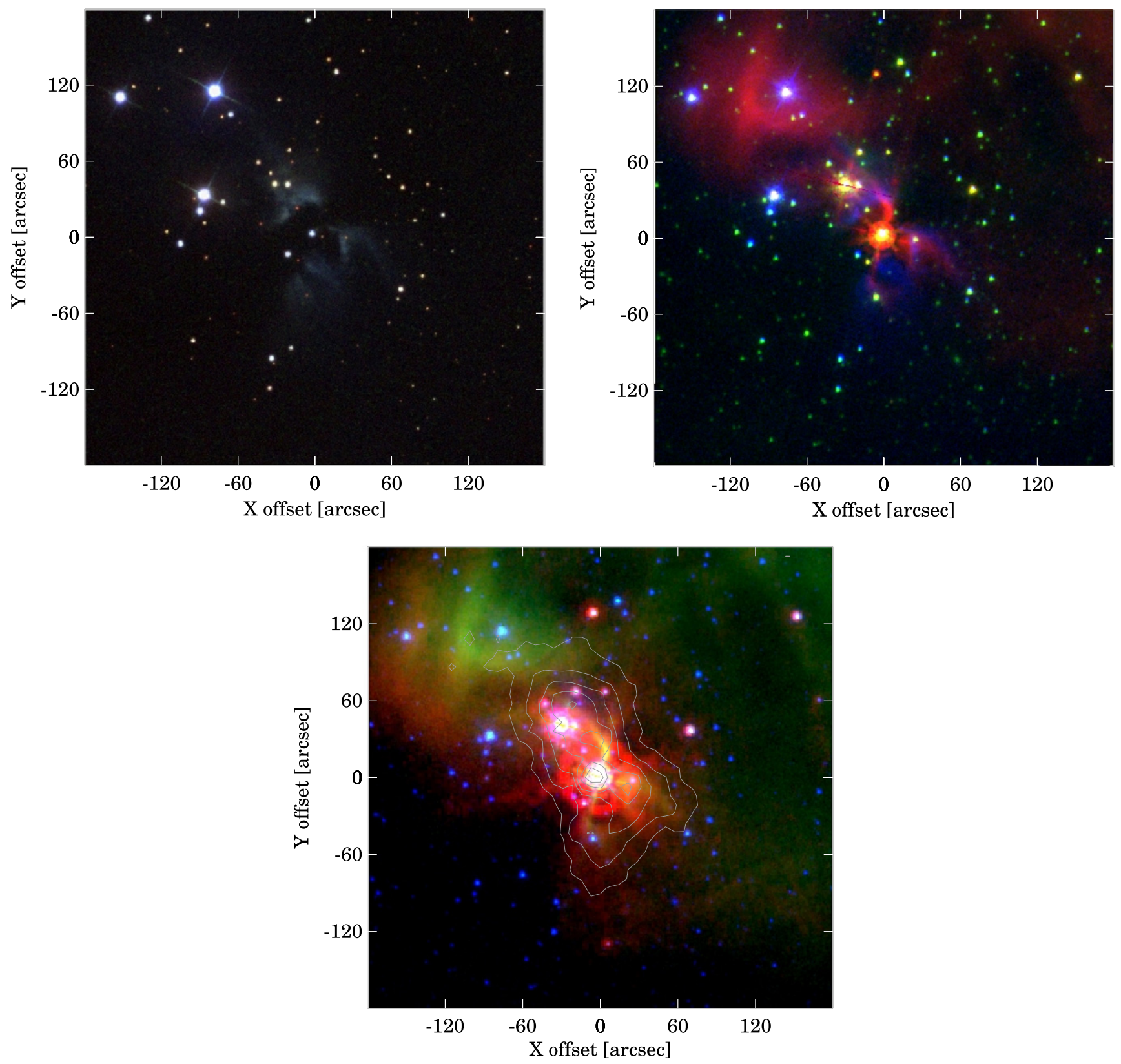

Figure 22. Upper left panel: optical three-color image of RNO 8, composed from the SDSS $g$ (blue), $r$ (green), and $i$ (red) images. Upper right panel: three-color image of the same region composed of SDSS $g$ (blue), IRAC $3.6 \mu \mathrm{m}$ (green), and IRAC $8 \mu \mathrm{m}$ (red) images. Lower panel: three-color image composed of the IRAC $4.5 \mu \mathrm{m}$ (blue), $8.0 \mu \mathrm{m}$ (green), and MIPS $24 \mu \mathrm{m}$ (red) images. Light gray contours show the distribution of the $70 \mu \mathrm{m}$ emission.

$0.1 M_{\odot} \leqslant M_{\text {star }} \leqslant 5.0 M_{\odot}$ interval are from Siess et al. (2000), and the track for $0.07 M_{\odot}$ from Baraffe et al. (2015) is also plotted. Most of the candidate YSOs are located between the $10^{6}$ and $10^{7} \mathrm{yr}$ isochrones, confirming their pre-mainsequence star nature at $825 \mathrm{pc}$ from us. Exceptions are a few Class II objects close to or below the ZAMS. The SEDs of these stars suggest that their disks have high inclinations, and thus most of the optical fluxes arise from scattered light (see Paper III for further details). The HRD suggests a mass range between $0.07 M_{\odot}$ (M5 type) and 2.5 $M_{\odot}$ (G- to early K-type stars, evolving toward higher $T_{\text {eff }}$ ).

We examined whether the average properties of stars surrounded by primordial (SED subtype II P in Table 9), weak
(II A), and transitional (II T) disks can be distinguished or not. Table 13 shows the mean $K_{\mathrm{s}}$ magnitudes and derived mean $A_{\mathrm{V}}$, $T_{\text {eff }}, L_{\mathrm{star}}, T_{\mathrm{bol}}$, and $L_{\mathrm{bol}}$ values of the three groups. The table shows that most of the candidate CTTSs of L1340 have weak (anemic) disks. We find that the central stars of primordial disks are brighter in each photometric band and have higher average $T_{\text {eff }}$ than the others, in accordance with the findings of Paper III. The bright $\mathrm{H} \alpha$ emission stars of Flat SED (Table 2, Paper III) fit into this trend.

A most prominent member of the $\mathrm{T}$ Tauri star population of the region is the $\mathrm{H} \alpha$ emission star associated with IRAS 02236 +7224 . Its early $G$ spectral type (Paper III) suggests a mass of $\sim 2 M_{\odot}$. The Omega-Cass images reveal a faint companion at 
an angular distance of 1 !! 12 ( $760 \mathrm{au})$. The IRAC $8 \mu \mathrm{m}$ image shows a further companion at 4". 3 (3550 au) to the northwest and another one at 4!9 (4040 au) to the southeast from the primary star (Figure 14, lower right panel).

\section{SURFACE DISTRIBUTION OF THE YOUNG STELLAR POPULATION}

The positions of all candidate YSOs, identified by infrared color indices, are overplotted on the extinction map of the region, together with ${ }^{13} \mathrm{CO}$ and $\mathrm{C}^{18} \mathrm{O}$ contours (from Paper I), in Figure 26. More detailed maps of the central regions of the

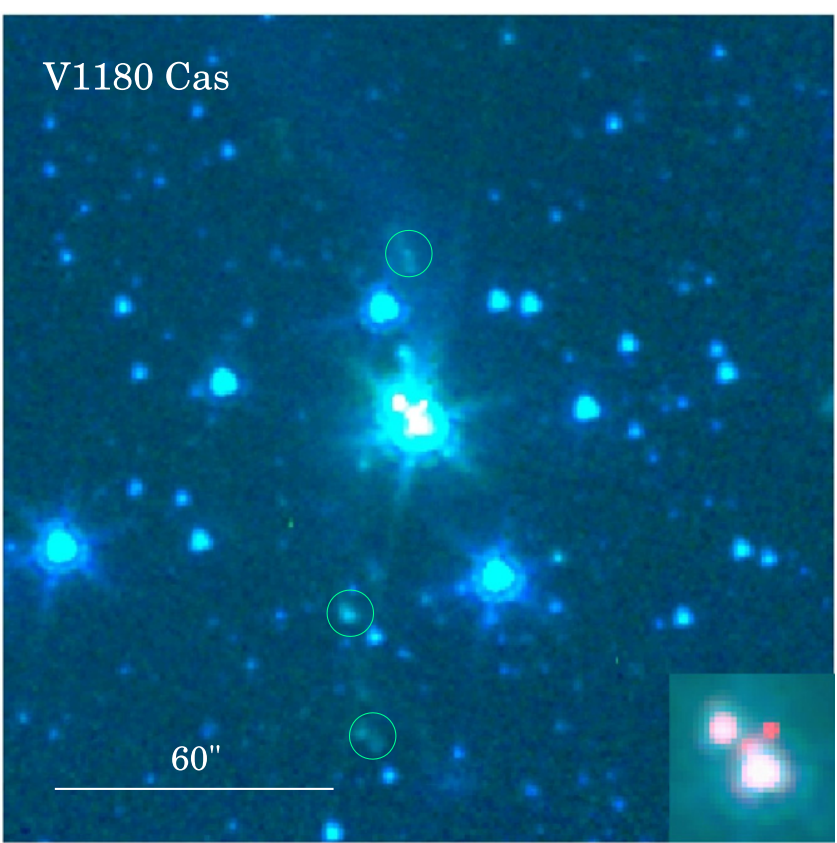

Figure 23. Same as Figure 20, but for the environment of V1180 Cas. In addition to the jet, emanating northward from the Class I component (Antoniucci et al. 2014), several fainter $\mathrm{HH}$ knots can be detected. A third component of the system emerges in the $8 \mu \mathrm{m}$ image. The inset shows an $18^{\prime \prime} \times 18^{\prime \prime}$ area of the central objects.
L1340 A, L1340 B, and L1340 C clumps are presented in Figures 27-29, respectively.

The surface distribution of the candidate YSOs reveals a rich population of young stars clustered over the clumps L1340 A, B, and C. We constructed surface density maps of the YSOs following the method described by Gutermuth et al. (2005). We determined the $r_{N}(i, j)$ distance of the $N$ th nearest star at each $(i, j)$ position of a uniform grid and obtained the local surface density of YSOs at the grid point as $\rho(i, j)=N / \pi r_{N}^{2}(i, j)$. The surface density contour plot, shown in the left panel of Figure 30, was constructed using a $30^{\prime \prime}$ grid and $N=6$ and shows the surface densities of Class I+Flat (red dot-dashed contours) and Class II (blue solid contours) sources separately, overlaid on the WISE $12 \mu \mathrm{m}$ image of L1340. The contour labels indicate the surface densities in star $\mathrm{pc}^{-2}$ units. The YSO groups associated with

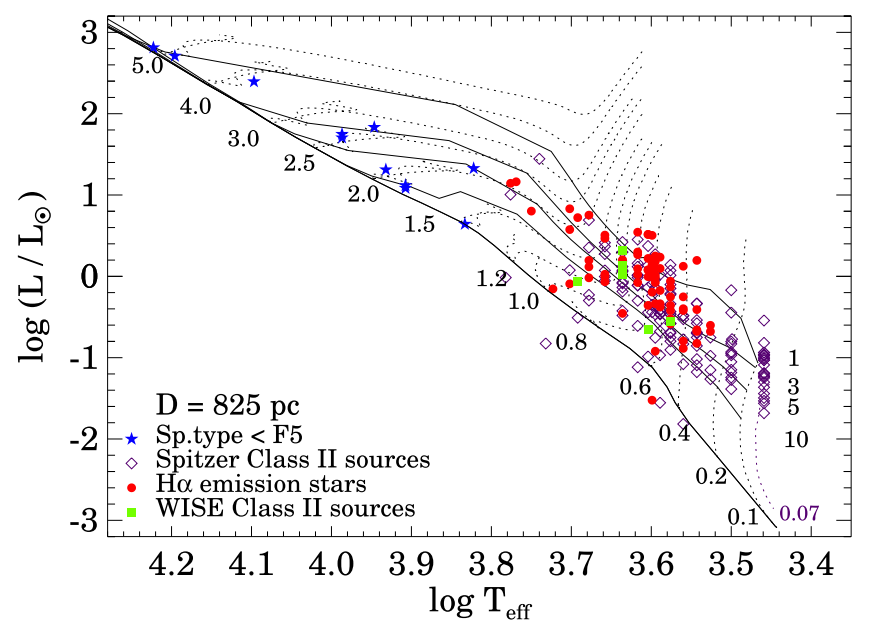

Figure 25. HRD of the candidate young population of L1340. Blue star symbols indicate the stars earlier than F5 (Paper III); red filled circles show Class II sources with $\mathrm{H} \alpha$ emission detected (Paper III). Open diamonds indicate Class II sources not detected during our $\mathrm{H} \alpha$ survey. Black dotted lines indicate evolutionary tracks, and thin solid lines mark the $1 \times 10^{6}, 3 \times 10^{6}$, $5 \times 10^{6}$, and $10^{7} \mathrm{yr}$ isochrones from Siess et al. (2000). The track for $0.07 M_{\odot}$ (purple dotted line) is from Baraffe et al. (2015). The bolometric luminosities of the selected sources were calculated for a distance of $825 \mathrm{pc}$.
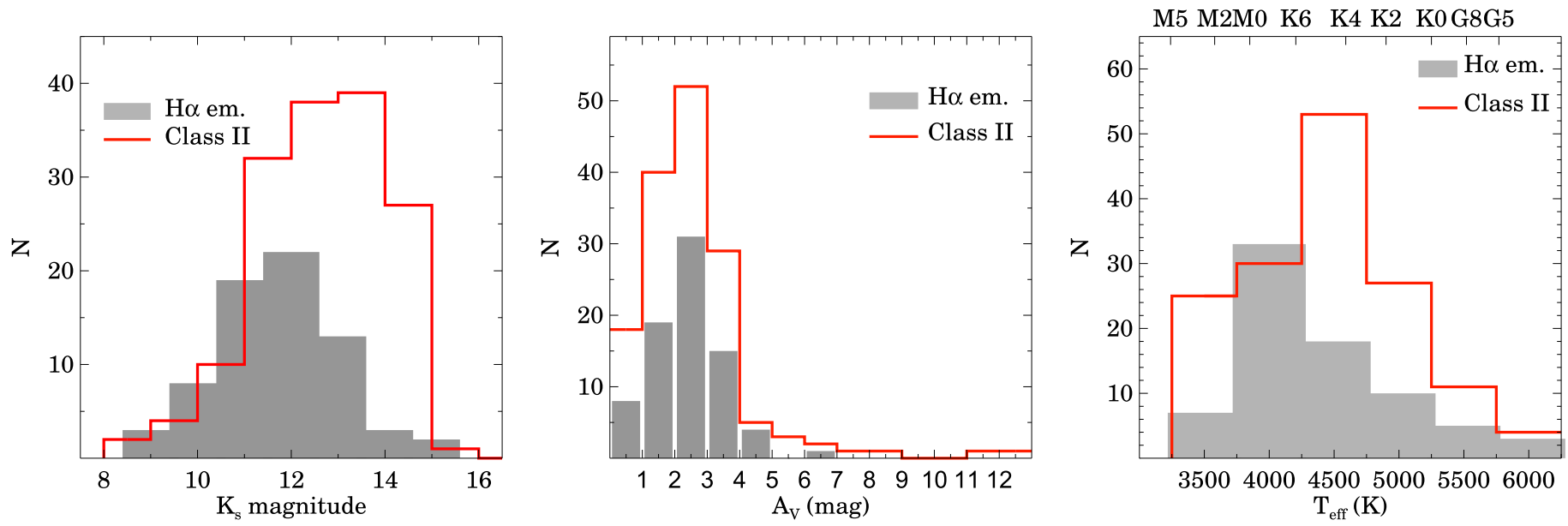

Figure 24. Histogram of the $K_{\mathrm{s}}$ magnitudes (left), visual extinctions (middle), and effective temperatures/spectral types (right), derived from photometric data of the candidate pre-main-sequence stars of L1340. 


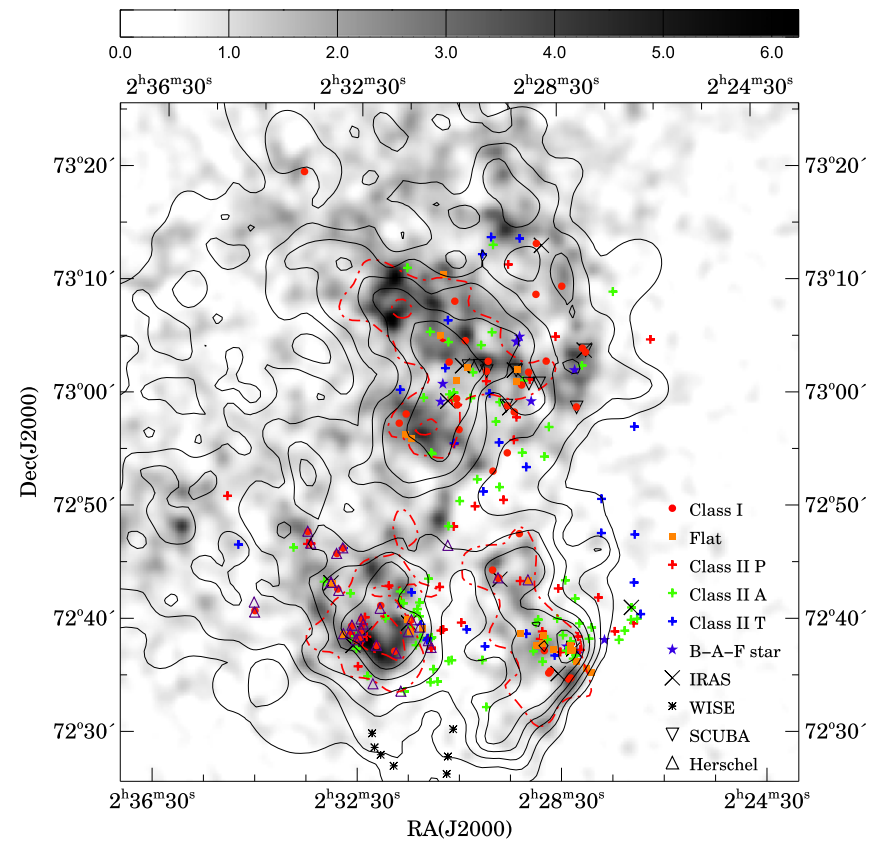

Figure 26. Surface distribution of the candidate YSOs, identified by infrared colors, overplotted on the visual extinction map of L1340, determined from star counts in the SDSS DR9 (Paper III). Black contours show ${ }^{13} \mathrm{CO}$ integrated intensities, drawn at $0.6,1.2,1.8, \ldots \mathrm{K} \mathrm{km} \mathrm{s}^{-1}$, and the red dot-dashed contour shows the $\mathrm{C}^{18} \mathrm{O}$ contour at $0.35 \mathrm{~K} \mathrm{~km} \mathrm{~s}^{-1}$. The meaning of the symbols is shown in the lower right corner.

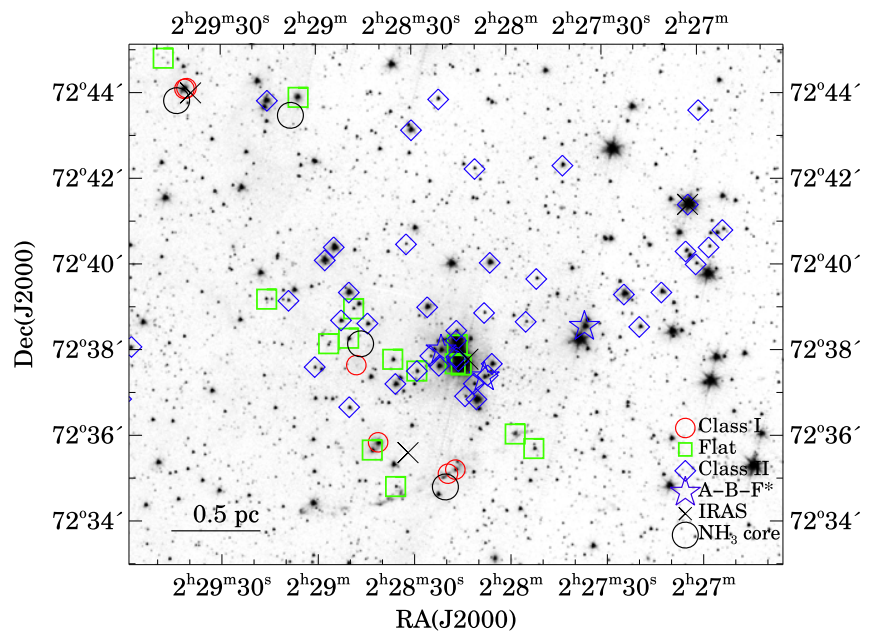

Figure 27. Surface distribution of the candidate YSOs and dense $\mathrm{NH}_{3}$ cores in a $15^{\prime} .6 \times 12^{\prime}$ area of L1340 A, plotted on the IRAC $3.6 \mu \mathrm{m}$ image of the field.

the cloud clumps are apparent. Like the three clumps, the associated YSOs show diverse surface structures. The surface distribution in L1340 A suggests a west-to-east progression of star formation. Similarly, in L1340 C, Class I and Class II sources are apparently separated from each other. The largest clump L1340 B is associated with an extended, low surface density population. The right panel of Figure 30 shows a composite surface density distribution of all YSO classes, derived at the same grid points, and using the distance of the twentieth-nearest YSO. The three clusterings, associated with the three clumps, remain apparent in the smoothed surface density map. The area of each YSO group and the number of

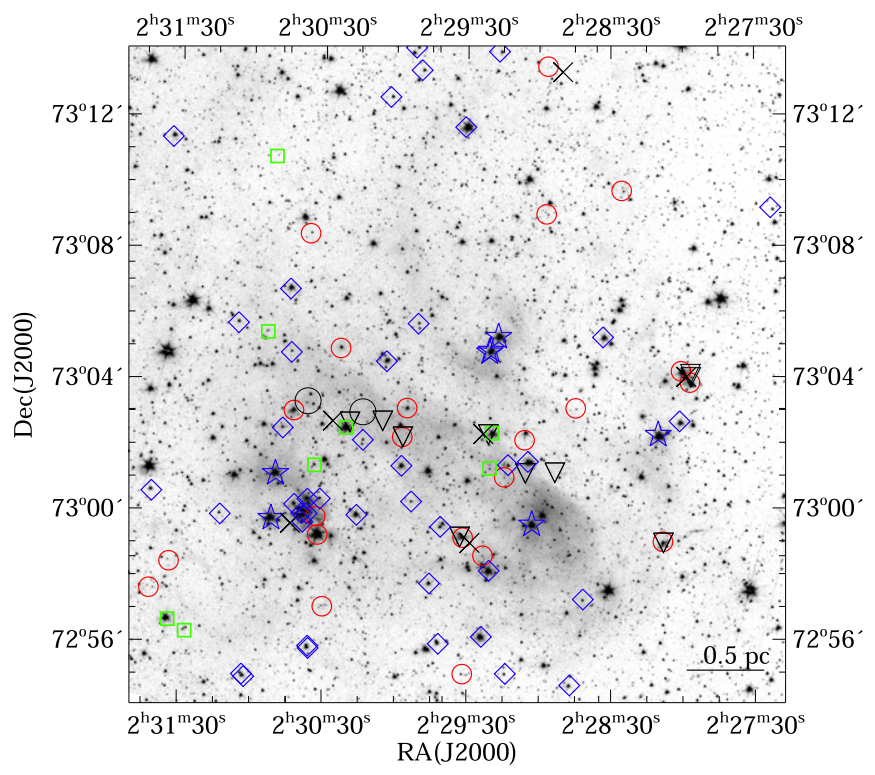

Figure 28. Surface distribution of the candidate YSOs and dense $\mathrm{NH}_{3}$ cores in a $20^{\prime} \times 20^{\prime}$ area of L1340 B. Symbols are the same as in Figure 27, plus downward-pointing triangles mark submillimeter sources.

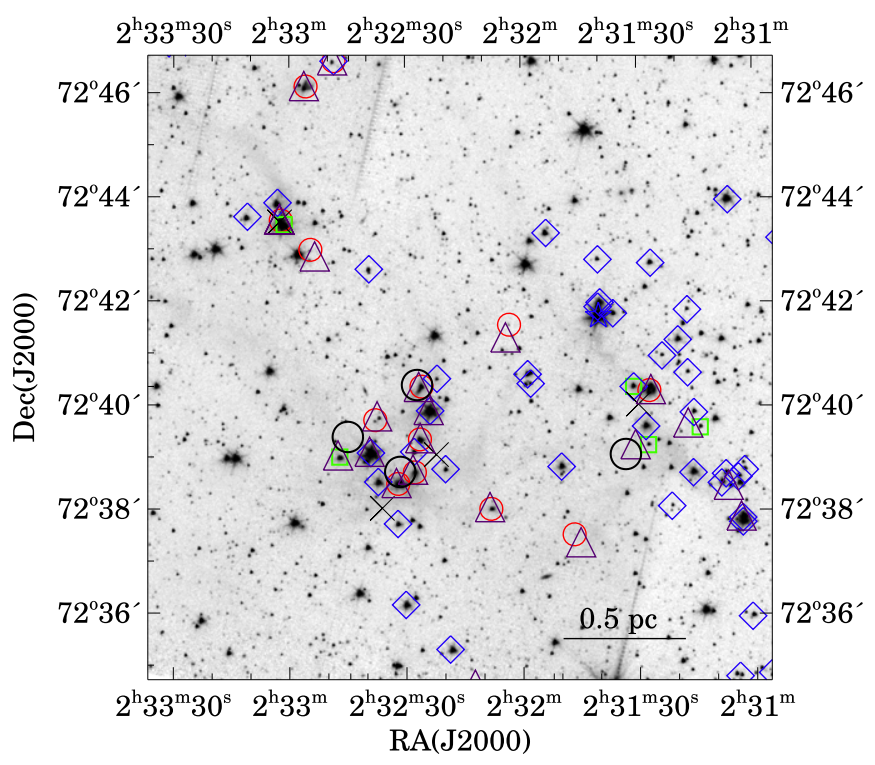

Figure 29. Surface distribution of the candidate YSOs and dense $\mathrm{NH}_{3}$ cores in a $12^{\prime} \times 12^{\prime}$ area of L1340 C. Symbols are the same as in Figure 27, plus triangles indicate far-infrared sources detected in the Herschel PACS images.

stars within the surface density contour $2 \mathrm{stars}_{\mathrm{pc}}^{-2}$ are listed in Table 14.

\subsection{Young Clusters in $L 1340$}

To find and characterize clusters in the YSO population of L1340, we examined the projected distances between the stars within the three groups seen in Figure 30. Figure 31 shows the histograms of the nearest neighbor separations for the three groups, separately. The histograms of groups associated with L1340 A and L1340 C show peaks at short spacings, similarly to other nearby SFRs (Gutermuth et al. 2009). On the contrary, no preferred spacing range can be seen in the histogram of 
Table 13

Average Properties of Candidate PMS Stars with Different SED Slopes

\begin{tabular}{|c|c|c|c|c|c|c|c|}
\hline SED Slope & $\left\langle K_{s}\right\rangle$ & $\left\langle A_{\mathrm{V}}\right\rangle$ & $\left\langle T_{\text {eff }}\right\rangle$ & $\left\langle L / L_{\odot}\right\rangle$ & $\left\langle T_{\text {bol }}\right\rangle$ & $\left\langle L_{\mathrm{bol}} / L_{\odot}\right\rangle$ & $N$ \\
\hline Primordial (II P) & 12.225 & 2.83 & 4160 & 1.02 & 2634 & 1.49 & 46 \\
\hline Evolved (II A) & 13.474 & 2.83 & 3620 & 0.69 & 2460 & 0.84 & 80 \\
\hline Evolved (II T) & 13.343 & 2.34 & 3660 & 0.34 & 2378 & 0.51 & 29 \\
\hline All Class II & 13.124 & 2.74 & 3806 & 0.71 & 2480 & 0.96 & 155 \\
\hline
\end{tabular}
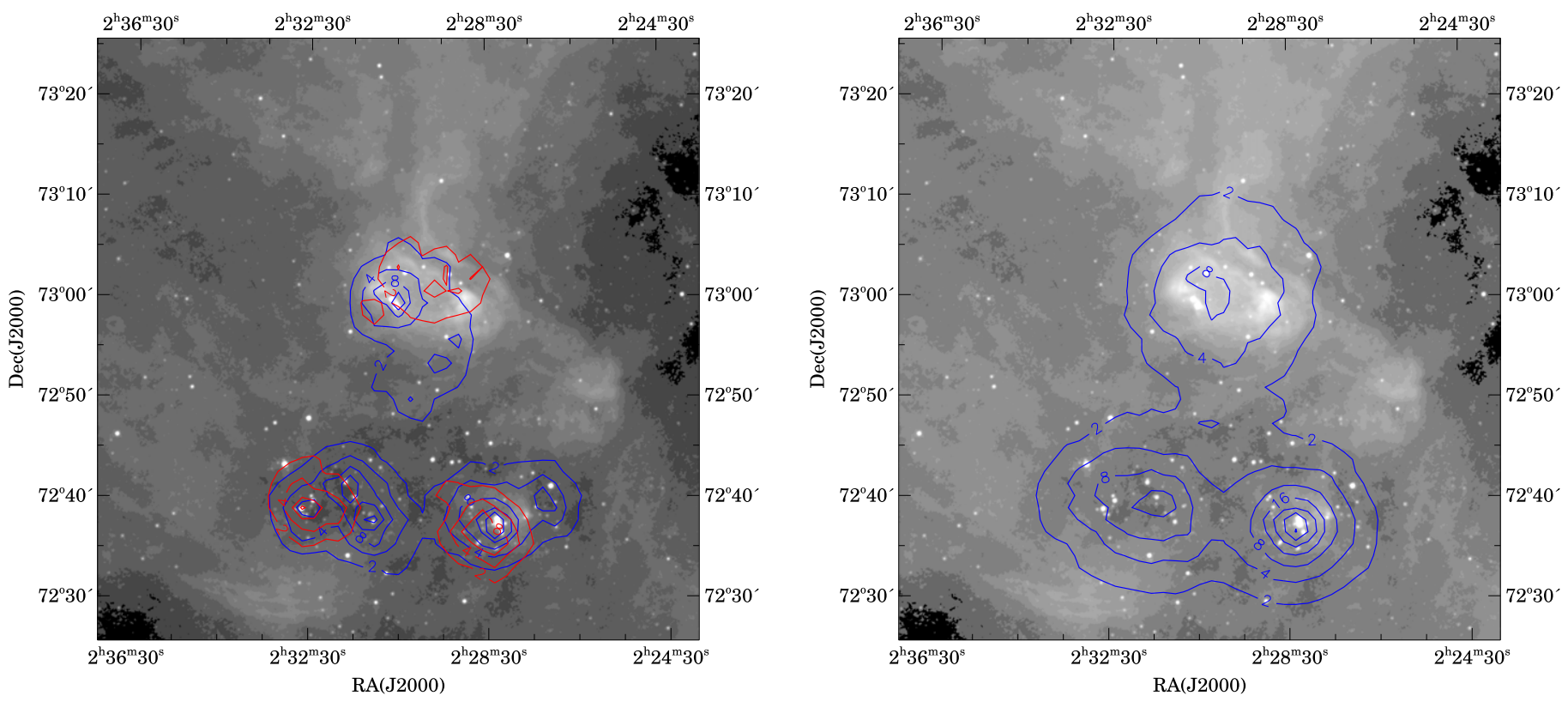

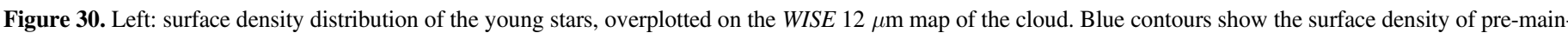

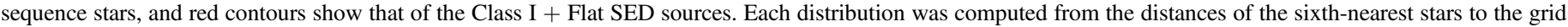

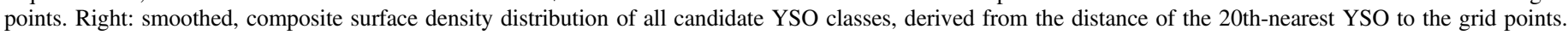

Table 14

Major Properties of the YSO Groups Associated with the Cloud Clumps

\begin{tabular}{lcccc}
\hline \hline Clump & L1340 A & L1340 B & L1340 C & $\begin{array}{c}\text { L1340 } \\
\text { Total }^{\mathrm{a}}\end{array}$ \\
\hline Area $\left(\mathrm{pc}^{2}\right)$ & 5.4 & 10.8 & 7.0 & $90^{\mathrm{b}}$ \\
$N($ Class 0/I) & 8 & 23 & 14 & 45 \\
$N($ Flat $)$ & 12 & 9 & 6 & 27 \\
$N($ Class II $)$ & 51 & 47 & 50 & 170 \\
$N($ Sp $\leqslant$ F5 $)$ & 3 & 7 & 1 & 11 \\
$N($ total $)$ & 74 & 87 & 71 & 253 \\
Mean surface density & 41.1 & 5.25 & 13.4 & 2.8 \\
$\quad\left(\right.$ stars pc $\left.^{-2}\right)$ & & & & \\
NII / (NI+NFlat) & 2.55 & 1.47 & 2.5 & 2.36 \\
SFE $^{\mathrm{c}}$ & 0.17 & 0.03 & 0.05 & 0.03 \\
\hline
\end{tabular}

Notes.

${ }^{\mathrm{a}}$ Stars outside of the surface density contour of 2 stars $\mathrm{pc}^{-2}$ are also included in this column.

b Total area of L1340 with $A_{\mathrm{V}}>1.0$ mag (Paper III).

${ }^{\mathrm{c}}$ Star formation efficiency, assuming average stellar mass of $0.5 M_{\odot}$, and taking the clump masses from Paper III.

L1340 B. The median separations of the YSOs are 0.117, 0.243 , and $0.141 \mathrm{pc}$ in L1340 A, B, and C, respectively.
Figure 32 shows the YSO distribution overplotted on the extinction map, and the stars having a neighbor closer than $0.15 \mathrm{pc}\left(\approx 42^{\prime \prime}\right)$ are marked by underlying black dots. In L1340 A, 75\% of the YSO population belongs to this clustered subsystem, while $66 \%$ of the YSOs in L1340 C and 34\% in L1340 B have neighbors within this distance. We identified four small clusters encircled by the overplotted ellipses. This criterion reveals 56 members of the RNO 7 cluster in L1340 A, including the $K$-band excess stars identified in the Omega-Cass data. The RNO 9 cluster in L1340 C consists of 1 Class I, 3 flat, and 22 Class II sources, whereas 6 of the 12 members of the cluster associated with IRAS $02276+7225$ are Class I/Flat sources. The only small clustering in clump B consists of eight stars, including the bright Class I source RNO 8. The coordinates and sizes of the clusters, identified by the nearest neighbor spacings, and the number of stars within them are listed in Table 15. The sizes are described by the major axis (a) and aspect ratio (AR) of the smallest ellipse encircling the members. The sampling of the members was not homogeneous, since part of L1340A was not covered by the MIPS observations, whereas the IRAS $02276+7225$ cluster is outside of the 4.5 and $8 \mu \mathrm{m}$ IRAC images. Moreover, eight members of the central core of RNO 7 come from the Omega-Cass observations. For comparison, the last row of Table 15 lists the 

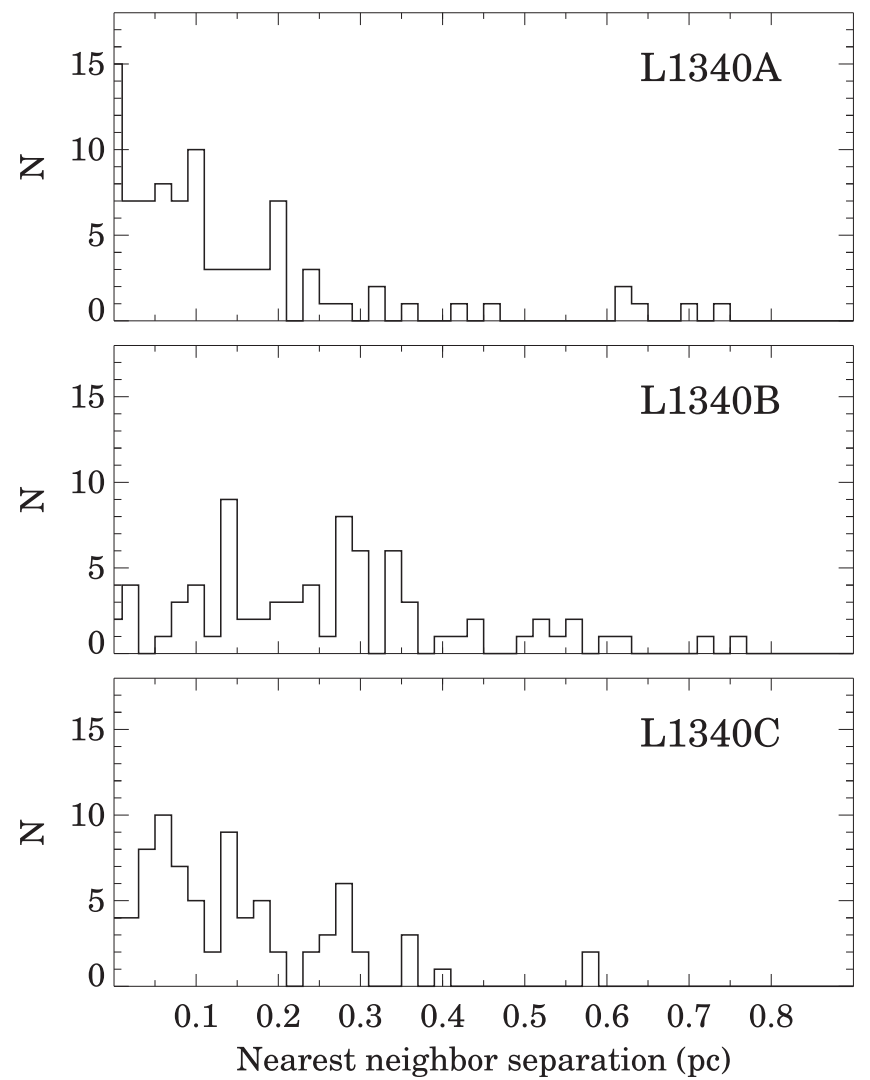

Figure 31. Histograms of the nearest neighbor separations for the three clumps of L1340.

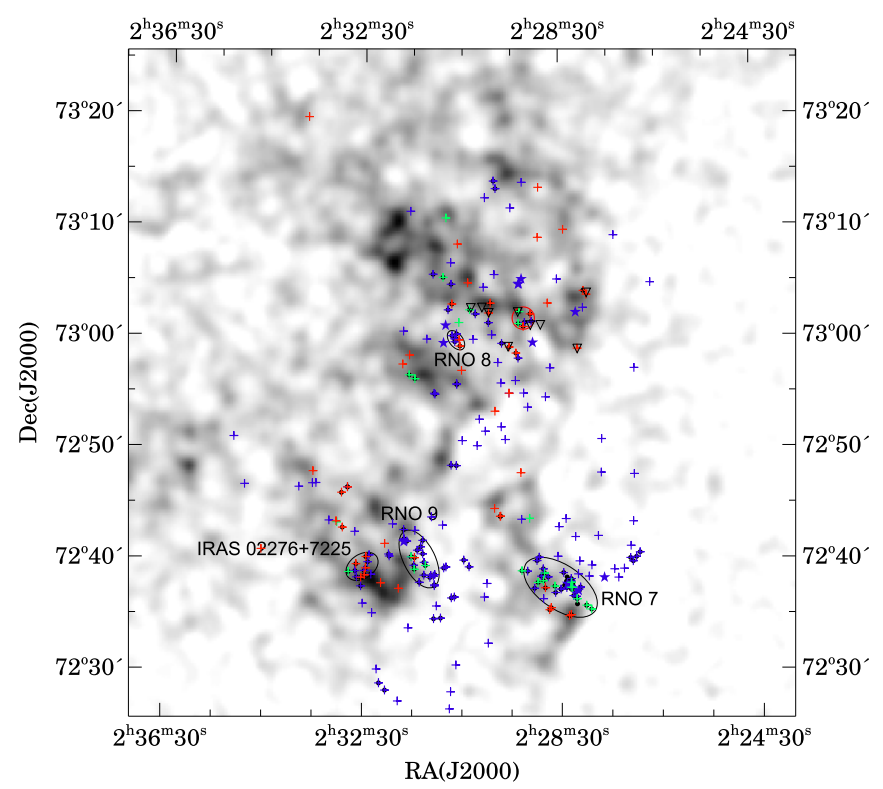

Figure 32. Distribution of candidate YSOs overplotted on the extinction map of L1340. Red symbols indicate Class I sources, green is for Flat, blue is for Class II YSOs, and triangles indicate the submillimeter sources. Sources having a neighbor at a projected separation smaller than $0.15 \mathrm{pc}$ are marked with underlying small black circles. Ellipses encircle the most prominent clusterings identified during this procedure. median values derived for the young cluster sample in our $1 \mathrm{kpc}$ Galactic environment (Gutermuth et al. 2009).

\subsection{YSO Distribution and the Cloud Structure}

The four small, compact clusters identified above comprise nearly half of the candidate YSOs. The distributed population consists of Class II stars scattered widely over low-extinction regions and small groups of a few closely spaced YSOs. An example is the small aggregate marked by a red circle in Figure 32, consisting of Class I, Flat, submillimeter, and strongly reddened Class II sources, and similar in angular size to the knots seen in the extinction map. To demonstrate the connection between the cloud structure and YSO distribution, we present in Figure 33 a multiwavelength view of L1340 B, revealing various aspects of interactions between the cloud and embedded stars. The upper panel of Figure 33 suggests that Class 0/I sources of L1340 B are associated with small-scale dust clumps. The morphology of this image suggests that the filamentary structure, detected at $850 \mu \mathrm{m}$, might have been created by past and present winds of the nearby young B- and A-type stars. The middle panel demonstrates the interactions of the intermediate-mass stars with the gas and dust and reveals a diversity of the embedded YSOs. The lower panel reveals that a chain of Class 0/I/Flat sources and two ammonia cores (Paper II) are lined up along a ridge of $850 \mu \mathrm{m}$ emission, starting with the Class 0 source J022808.60+725904.5 at the southwestern side, and stretching over a projected length of some $3 \mathrm{pc}$ to the Flat SED source $023042.36+730305.1$ at the northeastern end. The average separation of the protostars/ bright knots along the submillimeter filament, $\sim 1 ! 6$, corresponds to $\sim 0.4 \mathrm{pc}$ at $825 \mathrm{pc}$.

Linear configurations in the distribution of protostars are thought to result from fragmentation of dense molecular filaments (e.g., Teixeira et al. 2006). The separation of protostars along the filament is of the order of the Jeans length. Temperatures and densities derived from the ammonia mapping of L1340 (from Paper II) suggest a Jeans length of $\sim 0.14 \mathrm{pc}$ for the dense cores of L1340. The wide separation of the protostars along the submillimeter filament of L1340 B, as well as the large average spacing of the nearest neighbors throughout the clump, suggests a higher temperature of the ambient medium. The $\mathrm{NH}_{3}$ cores are probably the coldest regions of the cloud, embedded in a warmer gas, heated by the nearby B- and A-type stars.

Another conspicuous linear feature is the J-shaped configuration of YSOs in L1340 C (the IRAS 02276+7225 cluster; Figure 29). The average separation of the objects within that chain is $27 ! .9$, corresponding to $0.11 \mathrm{pc}$ at a distance of $825 \mathrm{pc}$. The total length of the chain is some $0.9 \mathrm{pc}$, suggesting that these stars have been formed from cores of $\sim 0.1 \mathrm{pc}$ in diameter. This coincides with the average size of the ammonia cores studied in Paper II and is the same as the Jeans length at $T_{\text {kin }} \approx$ $12.5 \mathrm{~K}$ and $n_{\mathrm{H} 2} \approx 1.29 \times 10^{4} \mathrm{~cm}^{-3}$, resulting from the $\mathrm{NH}_{3}$ observations. The cloud structure, underlying the observed distribution of the protostars, can be seen in the distribution of the cold dust, revealed by the Herschel SPIRE images (Figure 7). 
Table 15

Positions, Sizes, and Stellar Contents of the Clusters Embedded in L1340

\begin{tabular}{|c|c|c|c|c|c|c|}
\hline Object & R.A.(J2000) & Decl.(J2000) & $a(\mathrm{pc})^{\mathrm{a}}$ & $\mathrm{AR}^{\mathrm{b}}$ & $N($ total $)$ & $N($ Class II $) / N($ Class I $)$ \\
\hline RNO 7 & 022831.2 & 723734 & 0.8 & 1.9 & 56 & 2.5 \\
\hline RNO 8 & 023042.0 & 725950 & 0.21 & 1.17 & 8 & 2 \\
\hline RNO 9 & 023121.6 & 724008 & 0.55 & 1.8 & 26 & 5 \\
\hline IRAS $02276+7225$ & 023232.6 & 723902 & 0.34 & 1.48 & 12 & 1.0 \\
\hline $\mathrm{SSYSC}^{\mathrm{c}}$ median & $\ldots$ & $\ldots$ & 0.39 & 1.82 & 26 & 3.7 \\
\hline
\end{tabular}

Notes.

${ }^{a}$ Major axis of the smallest ellipse containing the member stars.

b Aspect ratio of the same ellipse.

c A Spitzer Survey of Young Stellar Clusters within $1 \mathrm{kpc}$ of the Sun (Gutermuth et al. 2009).

\section{STAR FORMATION IN L1340}

At a distance of $825 \mathrm{pc}$ and a latitude of $11^{\circ} .5, \mathrm{~L} 1340$ is situated some $160 \mathrm{pc}$ above the Galactic plane, in a low-density outer region of the molecular disk of our Galaxy. (The Swan is floating on the surface of the Galactic molecular disk.) The average hydrogen column densities of the three molecular clumps are about $2.5 \times 10^{21} \mathrm{~cm}^{-2}$, slightly lower than the mean column density of $4.38 \times 10^{21} \mathrm{~cm}^{-2}$, obtained by Lundquist et al. (2015) for IMSFRs in the outer Galaxy. The extinction map of L1340 (Paper III) reveals a shallow molecular cloud, spotted with dense knots of a characteristic size of a few arcminutes $(\sim 0.5 \mathrm{pc})$. YSOs are grouped on similar angular scales, and Class 0/I-Flat sources appear closely associated with extinction knots (see Figure 33), suggesting that star formation occurs in small groups, consisting of a few stars, and scattered over the surface of the cloud. The most massive star in L1340 is an optically visible B4-type star of some $5 M_{\odot}$, whereas the YSOs revealed by our present survey are in the $0.07 \lesssim M / M_{\odot} \lesssim 2.5$ mass interval.

The number of embedded sources, as well as their ratio to the more evolved pre-main-sequence stars in an SFR, is an indicator of evolutionary state. Myers (2012) established relations between Class II/Class I number ratios, as well as ages and birthrates of young stellar clusters, assuming a constant protostellar birthrate. The Class II/Class I ratio (Table 14) suggests an age of $10^{6} \mathrm{yr}$ and a birthrate of 200-300 protostars $\mathrm{Myr}^{-1}$. The three clumps of L1340 differ from each other in several respects. The effects of young intermediate-mass stars on the environment are conspicuous in L1340 B. The wispy structure of the $8 \mu \mathrm{m}$ emission, the bowshock-like structure associated with an A2-type star, and the double-peaked CO lines at the positions of the Planck Cold Clumps (Wu et al. 2012) associated with L1340 suggest violent swirling of the gas in this region. The low surface density of YSOs, compared to the other clumps of the cloud, indicates that the prestellar gas in L1340 B had higher temperature and lower density than in L1340 A and L1340 C, owing to the heating from the ambient B-type stars. The higher proportion of protostars in L1340 B (NII/(NI+NFlat) = 1.47) suggests that the average age of the YSO sample is lower in this clump than in the others. SFRs like L1340 B are probably more transient structures than centrally condensed young embedded clusters. Pfalzner et al. (2015) have found that only clusters and associations with initial central surface densities exceeding a few $\times 10^{3} M_{\odot} \mathrm{pc}^{-2}$ will be detected as clusters at ages longer than $5 \mathrm{Myr}$.
Assuming an average mass of $0.5 M_{\odot}$ for each candidate YSO, and including the intermediate-mass stars, discussed in Paper III, we find the star formation efficiencies $\left(\mathrm{SFE}=M_{\text {star }} /\left(M_{\text {star }}+M_{\text {cloud }}\right)\right.$ listed in Table 14 for the three clumps of L1340. It can be seen that while some $17 \%$ of the gas turned into stars in L1340 A, the SFE is only 3\% for L1340 B and also for the whole cloud. The actual SFEs are probably somewhat higher, since the low-mass diskless YSO population of L1340 is still unknown.

\subsection{Comparison with Other Star-forming Regions}

Comparison of our target cloud with IMSFRs, located in similar environments, may help to understand the interstellar processes, leading to star formation near the outer boundaries of the Galactic molecular disk. The short expected lifetime of L1340 (probably $\ll 5 \mathrm{Myr}$ ) suggests that similar SFRs may be rare in our Galactic neighborhood. A sample of 50 IMSFRs, studied by Arvidsson et al. (2010), contains objects similar in stellar content and total mass to L1340. Most of them are, however, more distant, and thus their detailed structures are still unrevealed. The Spitzer sample of young clusters in our Galactic neighborhood (SSYSC; Gutermuth et al. 2009) also contains several IMSFRs. Comparison of our results with several properties of this sample of young clusters is shown in Table 15. It suggests that the clusters identified in the YSO population of L1340 are similar in size, shape, and stellar content to the SSYSC average. The distribution of the projected YSO separations, however, suggests that the mode of star formation in L1340 B is quite atypical. The median nearest neighbor separations are significantly smaller in each of the SSYSC clusters than in L1340 B. Another atypical feature of L1340 is that conspicuous YSO groups are being formed in the smaller clumps, whereas the largest clump, associated with the highest-luminosity stars of the region, has a fragmented structure, associated with tiny groups of YSOs, scattered over the area of the clump.

A few IMSFRs in our $1 \mathrm{kpc}$ Galactic environment are also located at latitudes around $10^{\circ}$ or higher and are apparently not associated with giant molecular clouds. Well-known examples are NGC 7023 and NGC 7129, both located more than $100 \mathrm{pc}$ above the Galactic plane, and forming small clusters with the brightest stars of B3 type. These regions may have star-forming histories similar to L1340. Expanding supershells could create conditions of star formation at intermediate Galactic latitudes. Apparently none of these SFRs are associated with supershells; thus, some other process, such as infall of high-velocity clouds or Kelvin-Helmholtz instabilities arising at the shearing surface 

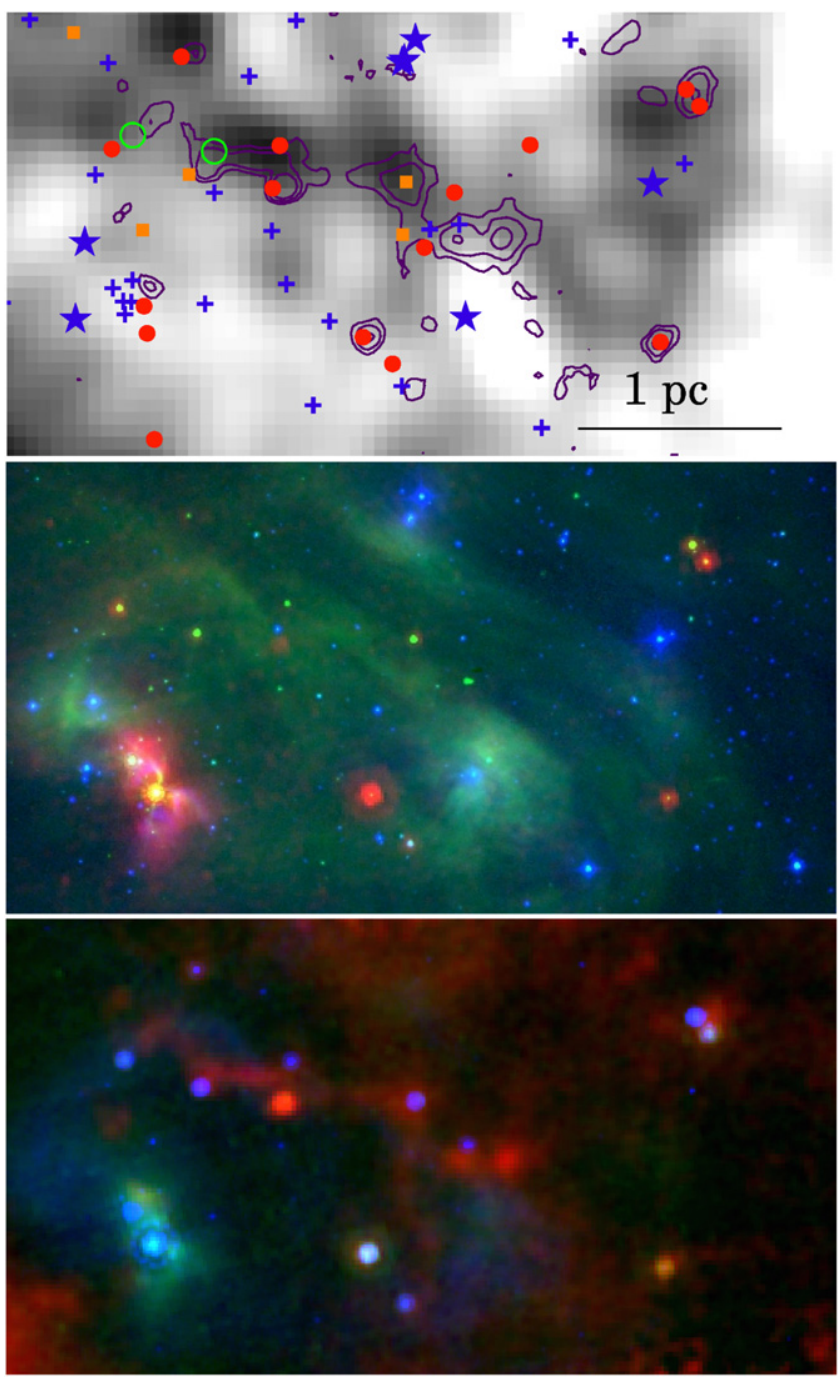

Figure 33. Multiwavelength view of the central, $17 ! 16 \times 9 ! 3$ area of the molecular clump L1340 B. The upper panel shows the positions of the B- to A-type stars (blue star symbols), Class 0/I infrared sources (red dots), flat SED (orange squares), Class II YSOs, ammonia cores (Paper II, green circles), and the contours of $850 \mu \mathrm{m}$ emission (purple contours, plotted at 30,60, and 120 $\mathrm{Jy}_{\text {beam }^{-1}}$ ), overplotted on the extinction map. Class I sources associated with tenth-of-parsec-scale density enhancements and a bubble-like cavity, bordered by submillimeter emission around an A-type star, can be seen. The middle panel is a three-color image of the same area, composed of DSS2 blue (blue), Spitzer IRAC $8 \mu \mathrm{m}$ (green), and MIPS $70 \mu \mathrm{m}$ (red) images. It reveals a bow-shock-like structure in the $8 \mu \mathrm{m}$ emission around an A-type star and the wispy structure of the diffuse $8 \mu \mathrm{m}$ emission. Brightness and color diversities of the embedded stars reveal their diverse evolutionary stages and luminosities. Lower panel: three-color image of the same region, composed of the MIPS $24 \mu \mathrm{m}$ (blue), MIPS $70 \mu \mathrm{m}$ (green), and SCUBA $800 \mu \mathrm{m}$ (red) images. The infrared sources lined up along the $\sim 2$ pc long, cold filamentary structure.

between gas layers of different velocities, might have compressed the gas.

\section{CONCLUSIONS}

We identified some 250 candidate YSOs associated with the moderate-mass $\left(\sim 3700 M_{\odot}\right.$; Paper III) molecular cloud L1340, based on Spitzer, AllWISE mid-infrared, and Omega-Cass near-infrared data, using various published color criteria. Supplemented with our measurements on the Herschel PACS
100 and $160 \mu \mathrm{m}$ images and publicly available photometric data, we constructed SEDs and classified 8 candidate Class 0 , 37 Class I, 27 Flat SED, and 170 Class II sources. Based on the SEDs, we derived extinctions and spectral types for the Class II sources and plotted them on the HRD. The HRD suggests a mass interval of $0.07-2.5 M_{\odot}$ for the CTTSs of our sample.

We identified new Herbig-Haro objects, associated with the Class 0 protostar SSTSL2 022808.60+725904.5 in the Spitzer images. The Spitzer data reveal that the bright IRAS source $02249+7230$ is a binary protostar associated with Herbig-Haro objects. The Spitzer data also suggest that the probable driving source of HH 488 is a Class 0 protostar, SSTSL2 J022820.81 +723500.5 . The projected length of $\mathrm{HH} 488$ is some $1.3 \mathrm{pc}$.

The Omega-Cass $J H K$ data resulted in eight new candidate members of RNO 7 and revealed a close companion of its brighest member, IRAS $02236+7224$. The Spitzer $8 \mu \mathrm{m}$ image revealed two further wide companions of this intermediatemass T Tauri star.

The surface density distribution of YSOs shows three groups, associated with the three major molecular clumps of L1340, each consisting of $\lesssim 100$ members, including both premain-sequence stars and embedded protostars. Based on the distribution of nearest neighbor separations, we identified four small clusters in the cloud, the RNO 7 cluster in L1340 A, RNO 8 in L1340 B, and RNO 9 and IRAS $02276+7225$ in L1340 C. Filamentary configurations of the protostars follow the distribution of the cold dust, traced by SCUBA and Herschel observations. The efficiency of the star formation in L1340 is some $3 \%$. Our results demonstrate that L1340 is a prolific SFR of our Galactic environment in which several specific properties of the intermediate-mass mode of star formation can be studied in detail. The distribution of dense gas and YSOs suggests that SFRs like L1340 are short-lived, transient objects.

This work is based on observations made with the Spitzer Space Telescope, which is operated by the Jet Propulsion Laboratory, California Institute of Technology, under a contract with NASA. This research utilized observations collected at the Centro Astronómico Hispano Alemán (CAHA) at Calar Alto, operated jointly by the Max-Planck Institut für Astronomie and the Instituto de Astrofísica de Andalucía (CSIC). This research has made use of the NASA/IPAC Infrared Science Archive, which is operated by the Jet Propulsion Laboratory, California Institute of Technology, under contract with the National Aeronautics and Space Administration. Our research has benefited from the VizieR catalog access tool, CDS, Strasbourg, France. Financial support from the Hungarian OTKA grant K81966 and K101393 is acknowledged. This work was partly supported by the Momentum grant of the MTA CSFK Lendület Disk Research Group.

\section{APPENDIX \\ $U B V R_{\mathrm{C}} I_{\mathrm{C}} J H K_{\mathrm{S}}$ PHOTOMETRIC DATA OF THE CLASS II YSOs}

We list $U B V R_{\mathrm{C}} I_{\mathrm{C}}$ magnitudes, transformed from the SDSS data, and 2MASS $J H K_{s}$ magnitudes of the color-selected candidate Class II young stars associated with L1340 in Table $\mathrm{A} 1$, excluding the $\mathrm{H} \alpha$ emission stars, whose data are given in Paper III. 
Table A1

$U B V R_{\mathrm{C}} \mathrm{I}_{\mathrm{C}} \mathrm{JHK}_{s}$ magnitudes of the Class II candidate YSOs of L1340, not detected as $\mathrm{H} \alpha$ emission stars

\begin{tabular}{|c|c|c|c|c|c|c|c|c|}
\hline Id & $\mathrm{U} \quad \mathrm{dU}$ & B $\mathrm{dB}$ & $\mathrm{dV}$ & $\mathrm{dR}_{\mathrm{C}}$ & $\mathrm{I}_{\mathrm{C}} \quad \mathrm{dI}_{\mathrm{C}}$ & $\begin{array}{ll}\mathbf{J} & \mathrm{d} \mathbf{J}\end{array}$ & $\mathrm{H} \quad \mathrm{dH}$ & $\mathrm{K}_{s} \quad \mathrm{dK}_{s}$ \\
\hline $022654.73+724040.8$ & 23.5411 .553 & 20.6270 .019 & 18.6710 .019 & 17.3710 .011 & 15.9060 .010 & 14.3380 .031 & 13.4610 .038 & 13.2290 .035 \\
\hline $022659.03+725716.0$ & 1.5110 .379 & 0.2460 .015 & 18.4800 .015 & 7.3320 .011 & 16.0040 .011 & 14.1300 .047 & 13.3120 .050 & 13.0740 .042 \\
\hline $022659.35+725714.2$ & 1.7820 .379 & 20.2460 .015 & 18.4830 .015 & 7.3010 .011 & 16.0220 .011 & 14.1300 .047 & 13.3120 .050 & 13.0740 .042 \\
\hline $022705.53+724116.7$ & 16.2280 .010 & 15.2880 .007 & 13.5680 .007 & 13.4430 .014 & 13.7150 .019 & 9.9010 .022 & 9.0840 .051 & 8.5790 .020 \\
\hline $022713.95+723913.8$ & 7.7860 .020 & 6.7780 .007 & 15.5030 .007 & 14.7050 .007 & 13.9220 .008 & 12.8760 .025 & 12.1570 .030 & 11.9380 .022 \\
\hline $2720.91+723825.6$ & 22.3130 .383 & 21.1140 .025 & 19.4830 .025 & 18.2860 .018 & 16.7240 .015 & 14.6960 .035 & 13.6540 .034 & 13.0680 .027 \\
\hline $22722.72+730913.8$ & 2.9340 .822 & 1.6750 .038 & 19.9710 .038 & 18.6830 .023 & 17.0140 .015 & 15.2870 .045 & 14.4980 .060 & 14.2140 .068 \\
\hline $022725.68+723911.1$ & 18.4460 .028 & 7.2940 .007 & 15.9240 .007 & 15.0650 .008 & 14.2210 .008 & 13.1670 .024 & 12.2340 .030 & 11.5260 .016 \\
\hline $022753.10+723933.2$ & 23.7360 .882 & 22.5020 .067 & 20.6680 .067 & 19.2210 .033 & 17.2490 .019 & 15.2890 .041 & 14.5370 .054 & 14.1830 .051 \\
\hline $022756.40+723833.2$ & 24.4441 .111 & 23.0940 .120 & 21.4670 .120 & 20.0720 .068 & 17.9420 .029 & 15.7070 .072 & 14.8490 .065 & $14.383 \quad 0.074$ \\
\hline $022807.66+723955.9$ & 20.5440 .096 & .0680 .009 & 17.3370 .009 & & 15.0950 .009 & 12.8650 .022 & 11.9970 .029 & 11.5780 .019 \\
\hline $022808.36+723718.1$ & 4.8991 .058 & .0150 .225 & .7870 .225 & & 18.0170 .032 & 5.7330. & 15.0860 .118 & 14.7080 .110 \\
\hline $022809.45+723845.6^{\mathrm{a}, \mathrm{b}}$ & 24.0451 .530 & .8280 .835 & 24.4610 .835 & 1630.8 & $\ldots$ & 17.1520 .217 & 16.1750 .214 & 15.1460 .130 \\
\hline $022812.42+724207.4$ & 24.7161 .106 & 3.7830 .201 & 22.0170 .201 & 4940. & 18.1690 .032 & 5.1290 & 14.3750 .051 & 430.049 \\
\hline $815.41+723649.0$ & 25.3831 .273 & 4.4400 .332 & .3940 .332 & 240. & 18.4080 .043 & 72 & 15.1860 .096 & 710.112 \\
\hline $318.15+723820.8$ & 25.1591 .092 & 25.0620 .465 & 22.9750 .465 & & 18.4470 .041 & 15.5430 .059 & 14.8130 .076 & 14.2920 .068 \\
\hline $22822.40+723808.4$ & 23.0460 .730 & 23.5200 .149 & 21.2880 .149 & 19.8880 .049 & & $15.036 \ldots$ & 15.9880 .168 & 14.6350 .089 \\
\hline $26.25+723745.4$ & 25.5831 .127 & 4.6500 .344 & 22.5810 .344 & 20.9320 .136 & 18.3090 .037 & 15.6610 .053 & 14.9090 .066 & 14.2730 .059 \\
\hline $2827.25+723853.4$ & 20.8540 .136 & .4200 .010 & 17.7590 .010 & 16.6440 .009 & 15.3770 .009 & 13.2430. & 12.1210 .030 & 11.5730 .020 \\
\hline $022830.47+723724.2^{\mathrm{a}, \mathrm{b}}$ & 22.9250 .974 & 3.0020 .130 & 21.0410 .130 & 19.8080 .059 & $\ldots$ & 16.7530. & 15.6270 .139 & 14.7810 .102 \\
\hline $022832.38+724301.7$ & 18.4450 .027 & 7.3380 .007 & 15.9800 .007 & 1300.0 & 14.2900 .008 & 13.3110. & 12.3920 .028 & 11.8740 .020 \\
\hline $833.91+724021.8$ & 23.9680 .7 & 00.2 & 590.2 & 370. & 18.4280 .0 & 220 & & \\
\hline 022 & 23.3460 .520 & 250. & .1350 .095 & & 17.8720 .027 & 5.7170 & 15.0920 .091 & 010.095 \\
\hline & 23.9950 .97 & 730. & 630.045 & 840 & 16.9090 .015 & 190 & 900.033 & 160.022 \\
\hline & 4.1990 .887 & 380.1 & 530.113 & 410 & 17.7450 .025 & 120 & 14.2820 .043 & 710.049 \\
\hline $.6^{\mathrm{b}}$ & 23.9391 .613 & 25.6110 .845 & 23.4480 .845 & 6990. & 18.8320 .074 & 7290. & 14.8150 .071 & 14.3480 .071 \\
\hline $022904.71+730131.0$ & 23.0180 .725 & 24.7670 .409 & 22.7820 .409 & & 19.3200 .082 & & & 12.4360 .039 \\
\hline $8+723902.3^{\mathrm{a}, \mathrm{b}}$ & 25.0831 .642 & 24.0170 .462 & 22.2250 .462 & 21.0550 .2 & $\ldots$ & 17.0250. & 0590.079 & 14.3500 .068 \\
\hline $022914.45+725503.6$ & 24.6601 .312 & 4.4630 .434 & 22.8520 .434 & & 18.5990 .054 & & 15.1300 .084 & 870.099 \\
\hline 0.6 & 24.5130 .681 & 30.392 & .4060 .392 & & 18.3900. & 6 & 14.5260 .047 & 900.062 \\
\hline $022937.11+725053.3$ & 18.3340 .024 & .1400 .007 & 6460.007 & 4.6950. & 13.7230 .008 & 2.2770. & 11.4900 .030 & 11.0880 .019 \\
\hline $022941.28+725932.4$ & 24.6991 .150 & 3.4930 .146 & 21.4360 .146 & 9.8740 .0 & 17.8340 .027 & 15.6140 .062 & 14.6900 .060 & 14.2490 .061 \\
\hline $022941.91+725201.7$ & 24.5560 .801 & 3.8070 .172 & 1.5230 .172 & .7850 .0 & 17.4930 .021 & .8970. & 4.2110 .0 & 13.7750 .036 \\
\hline & 3.5500 .988 & 9760.0 & 1770.045 & 520. & 16.7930 .015 & 120. & 50 & 490.047 \\
\hline & 3.3621 .0 & & 370. & & 16.9640. & & 47 & 490. \\
\hline & 3.6460 .6 & 470. & 380. & 270 & 990. & 610 & 01 & 640.099 \\
\hline & 21. & 6860. & 890. & 390 & 30. & 080 & 19 & 370. \\
\hline 18.6 & 770. & 40.238 & .1770 .238 & & 18.4840 .042 & 190. & 650.120 & 570.139 \\
\hline $.24+723234.4$ & 24.3151 .501 & & 00.071 & & 17.0680 .016 & & 41 & 30.027 \\
\hline 138.8 & 21.8590 .3 & 70.0 & 370.0 & 70. & 15.3510 .009 & & 12.5200 .034 & 30.027 \\
\hline 237.9 & 5.3521 .263 & 150.744 & 9670.744 & & 19.0130 .065 & & 14.8980 .067 & 30.065 \\
\hline $2.85+723643.4$ & 3.5690 .536 & 2.9760 .097 & 0.8810 .097 & 9.4170 .0 & 17.5020 .021 & & 14.1890 .048 & 13.7350 .046 \\
\hline $023003.62+730435.0$ & 19.3810 .045 & 1190.008 & 16.5490 .008 & 15.5400 .0 & 14.5180 .008 & 13.0550 .03 & 12.3020 .034 & 12.0300 .022 \\
\hline $023008.63+725243.2$ & 25.3681 .491 & 4.5010 .373 & 22.1920 .373 & & 18.0620 .036 & 15.8580 .0 & 15.0690 .079 & 14.5990 .084 \\
\hline & 5.4311 .274 & 1.8180 .435 & 2.8930 .435 & & 19.2220 .071 & 6.6500. & 15.5990 .114 & 14.9320 .108 \\
\hline & 3.7480 .7 & 340. & 390.1 & & 17.9 & & 86 & 670.097 \\
\hline & 1.0380 .0 & 560. & 90. & & 80. & & 47 & 500.041 \\
\hline 2.9 & 2.6560 .4 & 360. & 160. & & 00. & & 37 & 830.024 \\
\hline 48.0 & 26.6851 .216 & 25.4930 .703 & 23.1550 .703 & 21.3600 .237 & 18.9350 .069 & 15.9830. & 14.9310 .070 & 14.6320 .091 \\
\hline $023031.45+730023.6^{\mathrm{b}}$ & 23.0460 .730 & 23.5200 .149 & 21.2880 .149 & 19.8880 .049 & & 15.9690 .077 & 14.9040 .062 & 14.3310 .072 \\
\hline $023036.26+725550.5$ & 23.6290 .575 & 23.3450 .145 & 21.6710 .145 & 20.5470 .079 & 19.2810 .088 & 16.5280 .156 & 14.8460 .092 & 13.9390 .059 \\
\hline $23036.44+725554.5$ & 5.5451 .270 & 5.0660 .462 & 22.8210 .462 & . 2970.1 & 19.5390 .098 & - 5790.1 & 15.2200 .091 & 14.5160 .075 \\
\hline $023038.71+725941.2$ & 22.4161 .093 & 22.0240 .134 & 21.8060 .134 & 21.4260 .176 & 20.0240 .174 & 16.4400 .109 & 15.5780 .116 & 14.8780 .112 \\
\hline $23042.66+723638.9$ & 2.4150 .455 & .0760 .024 & 19.2010 .024 & 7.8350 .0 & 15.8910 .010 & 13.8720 .024 & 13.0400 .030 & 12.6440 .019 \\
\hline $023043.12+724834.4$ & 3.4520 .690 & 22.5770 .074 & 20.8330 .074 & 19.6460 .0 & 18.0990 .031 & 15.7260 .070 & 14.3920 .045 & 13.6820 .031 \\
\hline $023043.40+730451.3$ & 4.2320 .7 & 24.5780 .4 & 22.6510 .4 & 20.9980 .1 & 18.5560 .052 & 16.4080. & 15.4940 .108 & 15.1130 .124 \\
\hline $023043.85+730647.2$ & 18.7130 .0 & 17.3470 .0 & 15.7870 .0 & 14.8280. & 13.8580 .0 & 150. & 11.7430 .030 & 200.021 \\
\hline $023047.01+730233.2$ & 23.8251 .063 & 2920.0 & 20.4980 .059 & & 17.1460 .018 & & 14.3050 .037 & 13.9420 .049 \\
\hline $023055.66+723449.8$ & 25.1441 .269 & 23.4470 .147 & 21.3780 .147 & & 17.7070 .024 & & 14.8400 .081 & \\
\hline $023100.74+723555.3$ & 23.8100 .663 & 23.2980 .133 & 21.2660 .133 & 19.7630 .054 & 17.5540 .021 & 15.0320 .048 & 14.2070 .047 & 13.6410 .045 \\
\hline $023102.68+723844.4$ & 27.8851 .702 & 26.8300 .689 & 23.2690 .689 & 20.9220 .161 & 18.3440 .038 & 15.6320 .063 & 14.6870 .072 & 14.1260 .060 \\
\hline $023103.83+725503.0$ & 25.5141 .228 & 24.6780 .346 & 22.3770 .346 & 20.5880 .097 & 17.8790 .027 & 15.3630 .050 & 14.7500 .058 & 14.3960 .067 \\
\hline $023104.03+723446.1$ & 25.0691 .410 & 25.0260 .535 & 23.0180 .535 & 21.3390 .232 & 18.5680 .043 & 16.1300 .105 & 15.0780 .079 & 14.6400 .083 \\
\hline $023104.33+723836.1$ & 24.9721 .428 & 24.0730 .228 & 21.7970 .228 & 20.0950 .075 & 17.9000 .026 & 15.3620 .053 & 14.4220 .057 & 13.9880 .047 \\
\hline $023105.58+730545.1$ & 23.0521 .003 & 20.7420 .019 & 18.9160 .019 & 17.6700 .012 & 16.3030 .012 & 14.7230 .040 & 13.9700 .037 & 13.6770 .041 \\
\hline
\end{tabular}


Table A1

(Continued)

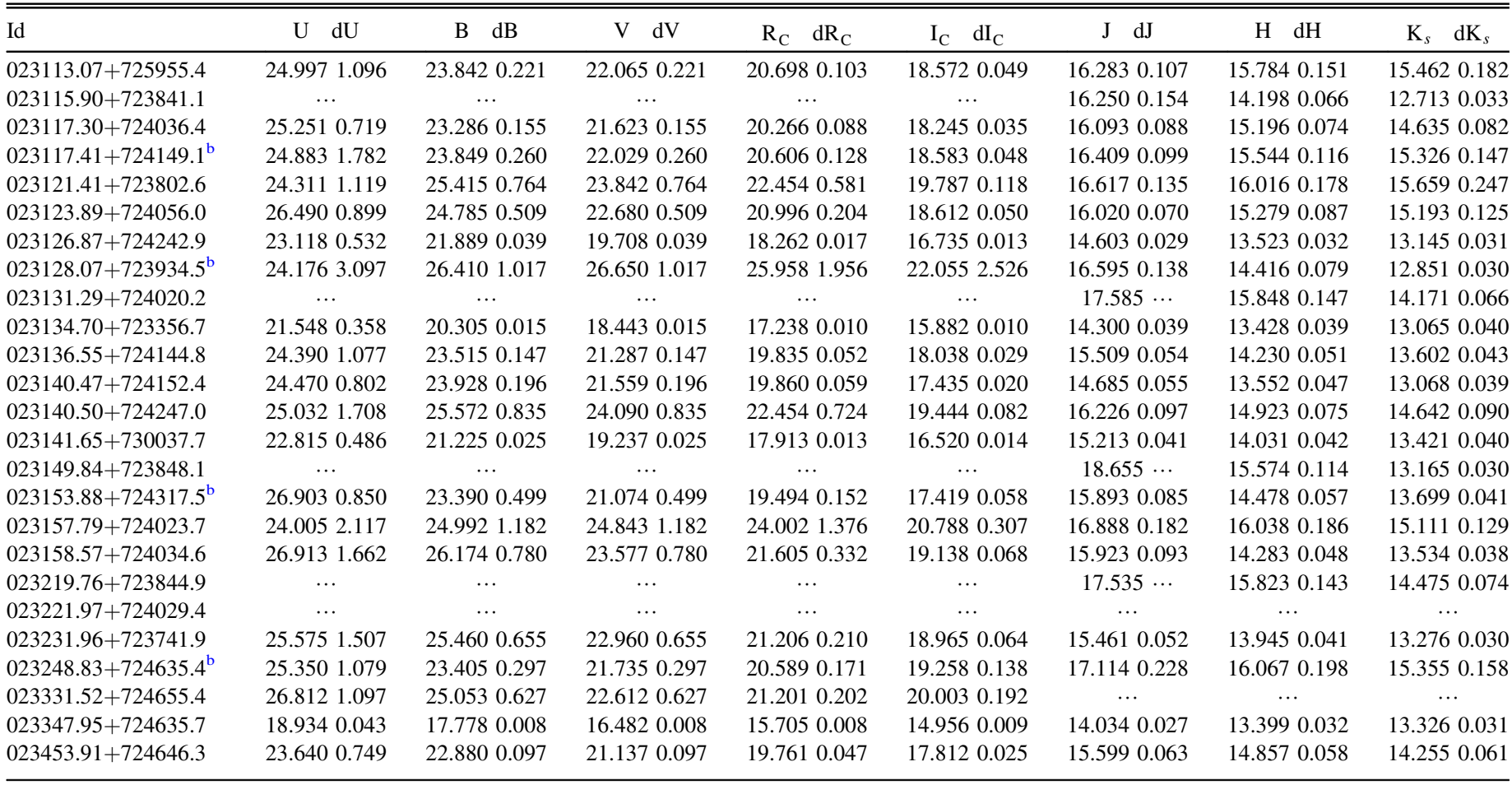

$U B V R_{\mathrm{C}} I_{\mathrm{C}}$ magnitudes were tarnsformed from the SDSS data, using the equations published by Ivezić et al. (2007) (for $B V R_{\mathrm{C}} I_{\mathrm{C}}$ ) and Jordi et al. (2006) (for $U$ ). $J H K_{\mathrm{s}}$ magnitudes are from the 2MASS All Sky Catalog of Point Sources (Cutri et al. 2003).

${ }^{a}$ UBVRI magnitudes of this star is transformed from the SDSS DR7 data.

${ }^{\mathrm{b}}$ SDSS classifies this object as a galaxy.

\section{REFERENCES}

Adams, F. C., \& Myers, P. C. 2001, ApJ, 553, 774

Ahn, C. P., Alexandroff, R., Allende, P., et al. 2012, ApJS, 203, 21

Antoniucci, S., Arkharov, A. A., Di Paola, A., et al. 2014, A\&A, 565, L7

Arce, H. G., Borkin, M. A., Goodman, A. A., Pineda, J. E., \& Beaumont, C. N. 2011, ApJ, 742, 105

Arimatsu, K., Doi, Y., Wada, T., et al. 2014, PASJ, 66, 47

Arvidsson, K., Kerton, C. R., Alexander, M. J., Kobulnicky, H. A., \& Uzpen, B. 2010, AJ, 140, 462

Balog, Z., Müller, T., Nielbock, M., et al. 2014, ExA, 37, 129

Baraffe, I., Homeier, D., Allard, F., \& Chabrier, G. 2015, A\&A, 577, A42

Bessell, M. S., \& Brett, J. M. 1988, PASP, 100, 1134

Chavarría-K, C. 1981, A\&A, 101, 105

Chen, H., Myers, P. C., Ladd, E. F., \& Wood, D. O. S. 1995, ApJ, 445, 377

Cohen, M. 1980, AJ, 85, 29

Cutri, R. M., Skrutskie, M. F., van Dyk, S., et al. 2003, yCat, 2, 246

D’Alessio, P., Calvet, N., Hartmann, L., et al. 1999, ApJ, 527, 893

Dib, S., Gutkin, J., Brandner, W., \& Basu, S. 2013, MNRAS, 436, 3727

Dickman, R. L. 1978, AJ, 83, 363

Di Francesco, J., Johnstone, D., Kirk, H., MacKenzie, T., \& Ledwosinska, E. 2008, ApJS, 175, 277

Doi, Y., Takita, S., Ootsubo, T., et al. 2015, PASJ, 67, 50

Dorschner, J., \& Gürtler, H. 1968, AN, 289, 65

Dunham, M., Allen, L. E., Evans, N. J., II, et al. 2015, ApJS, 220, 11

Enoch, M. L., Evans, N. J., II, Sargent, A. I., \& Glenn, J. 2009, ApJ, 692, 973

Evans, N. J., II, Calvet, N., Cieza, L., et al. 2009, arXiv:0901.1691

Fazio, G. G., Hora, J. L., Allen, L. E., et al. 2004, ApJS, 154, 10

Feigelson, E. D., Townsley, L. K., Broos, P. S., et al. 2013, ApJS, 209, 26

Furlan, E., Hartmann, L., Calvet, N., et al. 2006, ApJS, 165, 568

Greene, T. P., Wilking, B. A., André, P., et al. 1994, ApJ, 434, 614

Gutermuth, R. A., Megeath, S. T., Myers, P. C., et al. 2009, ApJS, 184, 18

Gutermuth, R. A., Megeath, S. T., Pipher, J. L., et al. 2005, ApJ, 632, 397
Harvey, P. M., Chapman, N., Lai, S.-P., et al. 2006, ApJ, 644, 307 Ishihara, D., Onaka, T., Kataza, H., et al. 2010, A\&A, 514, A1

Ivezić, Ž, Smith, J. A., Miknaitis, G., et al. 2007, in ASP Conf. Series, Vol. 364, The Future of Photometric, Spectrophotometric and Polarimetric Standardization, ed. C. Sterken (San Francisco, CA: ASP), 165

Jordi, K., Grebel, E. K., \& Ammon, K. 2006, A\&A, 460, 339

Juvela, M., Ristorcelli, I., Pagani, L., et al. 2012, A\&A, 541, A12

Kawada, M., Baba, H., Barthel, P. D., et al. 2007, PASJ, 59, 389

Koenig, X. P., \& Leisawitz, D. T. 2014, ApJ, 791, 131

Koenig, X. P., Leisawitz, D. T., Benford, D. J., et al. 2012, ApJ, 744, 130

Kryukova, E., Megeath, S. T., Gutermuth, R. A., et al. 2012, AJ, 144, 31

Kumar, M. S. N., Anandarao, B., \& Yu, K. C. 2003, AJ, 123, 2583

Kun, M. 2008, in ASP Monograph Publications, Vol. 4, Handbook of Star Forming Regions, Volume I: The Northern Sky, ed. B. Reipurth (ASP Monograph Publications), 240

Kun, M., Apai, D., O'Linger-Luscusk, J., et al. 2014, ApJL, 795, L26

Kun, M., Moór, A., Szegedi-Elek, E., \& Reipurth, B. 2016, ApJ, submitted (arXiv:1602.08120) (Paper III)

Kun, M., Obayashi, A., Sato, F., et al. 1994, A\&A, 292, 249 (Paper I)

Kun, M., Szegedi-Elek, E., Moór, A., et al. 2011, ApJL, 733, L8

Kun, M., Wouterloot, J. G. A., \& Tóth, L. V. 2003, A\&A, 398, 169 (Paper II)

Lada, C. J. 1991, in The Physics of Star Formation and Early Stellar Evolution, ed. C. J. Lada, \& N. D. Kylafis (Dordrecht: Kluwer), 329

Lundquist, M. J., Kobulnicky, H. A., Alexander, M. J., \& Arvidsson, K. 2014, ApJ, 784, 111

Lundquist, M. J., Kobulnicky, H. A., Kerton, C. R., \& Arvidsson, K. 2015, ApJ, 806, 40

Magakian, T. Yu., Movsessian, T. A., \& Nikogossian, E. G. 2003, Ap, 46, 1

Meyer, M. R., Calvet, N., \& Hillenbrand, L. A. 1997, AJ, 114, 288

Myers, P. C. 2012, ApJ, 752, 9

Myers, P. C., \& Ladd, E. F. 1993, ApJL, 413, L47 
Ott, S. 2010, in ASP Conf. Series, Vol. 434, Astronomical Data Analysis Software and Systems XIX., ed. Y. Mizumoto et al. (San Francisco, CA: ASP), 139

Pecaut, M. J., \& Mamajek, E. E. 2013, ApJS, 208, 9

Pfalzner, S., Vincke, K., \& Xiang, M. 2015, A\&A, 576, A28

Ridge, N., Wilson, T. L., Megeath, S. T., Allen, L. E., \& Myers, P. C. 2003, AJ, 126, 286

Rieke, G. H., Young, E. T., Engelbracht, C. W., et al. 2004, ApJS, 154, 25
Siess, L., Dufour, E., \& Forestini, M. 2000, A\&A, 358, 593

Teixeira, P., Lada, C. J., Young, E. T., et al. 2006, ApJL, 636, L45

Tóth, L. V., Marton, G., Zahorecz, S., et al. 2014, PASJ, 66, 17

Wright, E. L., Eisenhardt, P. R. M., Mainzer, A. K., et al. 2010, AJ, 140, 1868

Wu, Y., Liu, T., Meng, F., et al. 2012, ApJ, 756, 76

Yamamura, I., Makiuti, S., Ikeda, N., et al. 2010, yCat, 2, 298

Zhang, Y., \& Tan, J. C. 2015, ApJL, 802, L15 